\section{Pharmacology of Antimalarial Drugs, Current Anti-malarials}

Kesara Na-Bangchang ${ }^{1}$ and Juntra Karbwang ${ }^{2}$

${ }^{1}$ Chulabhorn International College of Medicine, Thammasat University, Pathumtanee, Thailand

${ }^{2}$ Clinical Product Development, Institute of

Tropical Medicine, Nagasaki, Japan

Currently available antimalarial drugs can be classified into four broad categories according to their chemical structures and modes of action.

1. Arylamino alcohol compounds: quinine, quinidine, chloroquine, amodiaquine, mefloquine, halofantrine, piperaquine, and lumefantrine

2. 8-Aminoquinoline: primaquine and tafenoquine

3. Antifolate compounds: sulfadoxine, pyrimethamine, proguanil, chlorproguanil, and trimethoprim

4. Artemisinin compounds: artemisinin, artesunate, artemether, $\beta$-arteether, and dihydroartemisinin

5. Others: atovaquoneand antibacterial drugs (tetracycline, doxycycline, and clindamycin)

\section{Arylamino Alcohol Antimalarials}

\section{Chloroquine}

Chloroquine was first synthesized by Bayer in Germany as early as 1934. The initial clinical studies were performed by German scientists just before World War II. However, the drug was reported to be too toxic for human use and not introduced for general use at that time. By late 1944, in the intensive search for an effective antimalarial drug during World War II, US workers synthesized 25 different 4 -aminoquinoline derivatives, with the objective of discovering more effective and less toxic suppressive agents than quinacrine. Of these compounds, chloroquine proved the most promising and later underwent extensive clinical studies. Since then, chloroquine had been used as the drug of choice for treatment of human malaria all over the world until the advent of chloroquine resistance in Plasmodium falciparum in the early 1960s. Clinical treatment failures of $P$. falciparum were first noted in Thailand almost at the same time as in South America. Chloroquine-resistant $P$. falciparum has since then spread relentlessly to virtually all areas of the world except Central America, North Africa, and parts of Western Asia.

\section{Chemistry and Physical Properties}

Chloroquine [7-chloro-4-(4-diethylamino-1-methylbutylamino) quinoline: Fig. 1a] is a weakly basic tertiary amine synthetic antimalarial agent which is a 4-aminoquinoline derivative. It has a quinoline ring with a side chain identical with that of quinacrine. The chlorine atom in the seventh position appears to be crucial to the antimalarial activity of all 4-aminoquinoline antimalarials. The drug is used 


\section{Pharmacology of Antimalarial Drugs, Current Anti-malarials,}

Fig. 1 Chemical structures of (a) chloroquine and

(b) mono-desethyl

chloroquine

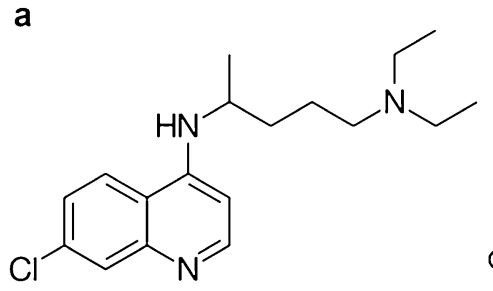<smiles>CC(CCCN)Nc1ccnc2cc(Cl)ccc12</smiles>

as a racemic mixture of equal amounts of $\mathrm{S}(+)$ and $\mathrm{R}$ (-) chloroquine.

Chloroquine is a white or slightly yellow, odorless, crystalline powder with a bitter taste. It is poorly soluble in water, but soluble in diluted acid, chloroform, and ether. Chloroquine phosphate is readily soluble in water at acidic $\mathrm{pH}$. Chloroquine sulfate is soluble in a mixture of water and methanol but non-soluble in pure water. The drug is sensitive to light and should be protected from light. The molecular weights of the base, phosphate, sulfate, and hydrochloride salts are 320, 516, 436, and 393, respectively. Structure-activity relationship (SAR) studies of many derivatives of the 4-aminoquinolines show that halogen substitutions at any position other than seven reduce pharmacologic activity and toxicity. An aryl rather than an alkyl side chain decreases the therapeutic ratio. Increasing alkyl side chain length above five carbons decreases the therapeutic ratio and increases toxicity.

\section{Pharmacological Activities}

Antimalarial Activity and Mechanism of Action and Resistance Chloroquine is highly effective and acts rapidly against asexual erythrocytic forms of Plasmodium vivax, Plasmodium ovale, Plasmodium malariae, Plasmodium knowlesi, and chloroquine-sensitive P. falciparum. (-)-Chloroquine is less active than $(+)$-chloroquine enantiomer against chloroquine-resistant strains of $P$. falciparum. Chloroquine is also active against gametocytes of $P$. vivax, $P$. malariae, and $P$. ovale and immature forms of $P$. falciparum. The drug has no effect against the exoerythrocytic tissue stages of malaria. Its major plasma metabolite monodesethylchloroquine (Fig. 1b) has similar antimalarial activity against chloroquine-susceptible parasites in vitro, but it is less effective than the parent compound against chloroquine-resistant strains.

The mechanisms of action and resistance of chloroquine have not been fully elucidated. Proposed mechanism(s) of action include DNA binding, inhibition of various enzymes (e.g., mixed-function oxidase, heme polymerase, phospholipase, and glutathione-S-transferase) and/or transporters, inhibition of protein synthesis, interference with digestion of host-derived hemoglobin in the parasite digestive food vacuole (acid lysosome), and lysosomotropic effect. Interference with hemoglobin digestion process and alteration of lysosomal $\mathrm{pH}$ appear to be important mechanisms of chloroquine action. During the process of hemoglobin digestion, the protein moiety of hemoglobin is degraded to related peptides, and heme is transformed into hemozoin (HZ), a nontoxic crystalline polymer. Chloroquine is a weak base with pKa values of 8.1 and 10.2 and the protonation of the drug encharged at the neutral $\mathrm{pH}$ of the blood. With the acidic $\mathrm{pH}$ for parasite food vacuole, chloroquine accumulates and binds hematin, a toxic product of hemoglobin degradation, therefore preventing its incorporation into the hemozoin crystal. The free hematin interferes with the parasite detoxification processes and thereby damages the Plasmodium membranes by lipid peroxidation mechanism.

Resistance to chloroquine in $P$. falciparum involves mainly the mutation in the parasite transport gene pfcrt (Plasmodium falciparum chloroquine resistance transporter) along with $p f m d r 1$ (Plasmodium falciparum multidrug resistance 1) and pfmrp1 (Plasmodium falciparum multidrug resistance-related protein 1). The pfcrt gene is located on chromosome 7 and encodes a $49 \mathrm{kDa}$ protein (PfCRT) localized in parasite's food vacuole with ten predicted transmembrane domains. 
An amino acid substitution at position 76 from lysine to threonine (K76 T), located on the first transmembrane domain, has been reported to be directly associated with chloroquine-resistant $P$. falciparum isolates in wide geographic areas. The $p$ fmdrl gene is located on chromosome 5 and encodes a homologue of the mammalian multidrug resistance gene in $P$. falciparum, P-glycoprotein homologue 1 (Pgh-1). Pfmdrl can modulate the degree of chloroquine resistance in some parasite strains, suggesting that some alleles and overexpression of PfMDR1 may enhance chloroquine concentration within the digestive food vacuole by active transport. The pfmrp 1 is located on chromosome 1 and encodes a 1822 amino acid proteins PfMRP1 which is a transporter member of the ATP-binding cassette (ABC) proteins similarly to PfMDR1. It is localized to the parasite plasma membrane. PfMRP1 modifies drug responses but is not a major determinant of chloroquine resistance. Potential inhibitors of these parasite transport proteins which could effectively reverse chloroquine-resistant $P$. falciparum in clinical settings are being investigated.

\section{Other Pharmacological Activities and Clinical}

Uses Apart from malaria, chloroquine is commonly used in patients with several inflammatory conditions, such as rheumatoid arthritis, systemic lupus erythematosus (SLE), discoid lupus erythematosus, porphyria cutanea tarda, polymorphous light eruptions, solar urticaria, recurrent basal cell carcinoma of the skin, porphyria cutanea tarda, and antiphospholipid antibody syndrome. In the treatment of amoebic liver abscess, chloroquine may be used instead of, or in addition to, other medications in case of unsatisfactory treatment efficacy or intolerance following metronidazole or another nitroimidazole within 5 days, as well as in case of intolerance to metronidazole or a nitroimidazole. The mechanism of action may involve its inhibitory effect on pro-inflammatory cytokine release into human whole blood. This effect may be of therapeutic benefit not only during chronic inflammation but also in diseases that are related to infection-induced inflammation.
Chloroquine has potential for use as an adjunct therapy with standard antiretroviral drugs. Synergistic activities have been demonstrated when chloroquine is used in combination with zidovudine, didanosine, and the protease inhibitors (indinavir, ritonavir, and saquinavir). Chloroquine is associated with low levels of HIV RNA in breast milk of the HIV-infected patients. In addition to HIV, inhibitory activity against the replication of severe acute respiratory syndrome (SARS) or coronavirus infections is shown in vitro.

Chloroquine also has potential for use as a chemosensitizer in cancer in conjunction with some conventional anticancer drugs, through inhibition of the function of membrane-associated proteins belonging to the $\mathrm{P}$-glycoprotein and multidrug resistance (MDR) protein families.

Chloroquine is a potent autophagic drug that may lead to cellular degradation of hepatocytes in the liver with the concurrent production of vacuoles.

Chloroquine has been shown to inhibit glucose 6-phosphate dehydrogenase activity in vitro. Administration of chloroquine to rats also caused alterations in several hepatic and renal antioxidant enzymes, thereby inducing an oxidative stress in these organs.

\section{Therapeutic Indications for Malaria}

Chloroquine is one of the most successful and widely used medications and with obvious health precautions, saving countless lives from malaria since the 1940s. The drug was for several decades the antimalarial of choice because it was effective, well tolerable, safe, and low cost. However, its usefulness has rapidly declined in most malariaendemic areas of the world where chloroquineresistant strains of $P$. falciparum and $P$. vivax have emerged and widespread. P. falciparum chloroquine resistance was first suspected in the late 1950s and confirmed in 1959 in Thailand. Almost simultaneously, resistance occurred in an independent focus in Colombia and South America. The use of chloroquine for treatment of $P$. falciparum infection is currently restricted to few countries in Caribbean and Central America, and the World Health Organization (WHO) 
strongly recommends artemisinin-based combination therapies (ACTs) for all $P$. falciparum confirmed cases in all endemic areas. Nevertheless, an increase in chloroquine susceptibility has been reported after withdrawal of the drug as first-line treatment, indicating that in the absence of the drug pressure, it is possible to restore chloroquine efficacy. The efficacy of chloroquine in the treatment of $P$. vivax and other non- $P$. falciparum is however still very high in most areas of the world including Thailand. Chloroquine-resistant P. vivax was first reported from Papua New Guinea in 1989, almost 30 years after the emergence of $P$. falciparum resistance. It is now widespread and has rendered this drug ineffective in parts of Indonesia and Papua New Guinea. Low levels of resistance have also been reported from Myanmar, South Korea, Vietnam, India, Turkey, Ethiopia, and parts of southern Africa and South America. Currently, chloroquine (or ACTs) is a therapeutic option for the treatment of adults and children with uncomplicated $P$. vivax, $P$. ovale, $P$. malariae, or $P$. knowlesi in areas with chloroquine-sensitive infections. Its use in combination, or not, with primaquine, the unique antimalarial drug that acts against the Plasmodium liver stage preventing relapses, is the first choice in the majority of endemic areas of these non- $P$. falciparum infections. Oral chloroquine is given at an initial dose of $10 \mathrm{mg} / \mathrm{kg}$ body weight (bw), followed by $10 \mathrm{mg} / \mathrm{kg}$ bw on the second day and $5 \mathrm{mg} / \mathrm{kg}$ bw on the third day (total dose of $25 \mathrm{mg} / \mathrm{kg} \mathrm{bw}$ ). In the past, the initial $10 \mathrm{mg} / \mathrm{kg}$ bw dose was followed by $5 \mathrm{mg} / \mathrm{kg}$ bw at 6,24 , and $48 \mathrm{~h}$. Chloroquine phosphate is available orally as tablets containing either 250 or $500 \mathrm{mg}$ of the phosphate (equivalent to 150 and $300 \mathrm{mg}$ of base, respectively).

Chloroquine has been considered safe when used in normal therapeutic doses during pregnancy. No abortifacient or teratogenic effects have been reported.

\section{Adverse Reactions and Toxicity}

At therapeutic doses, chloroquine is generally well tolerated. Individual variations in the pattern of distribution of chloroquine and its metabolite in the body are suspected to play an important bearing on the incidence and pattern of adverse reactions to the drug. Large doses used for the treatment of rheumatoid arthritis are associated with a higher frequency of adverse reactions than the lower doses used in malaria. The main adverse reactions reported after therapeutic or prophylactic regimens include abdominal discomfort (nausea, vomiting, and diarrhea), headache, blurred vision, light-headedness, and fatigue. Gastrointestinal disturbance can be minimized by taking the drug with food. Ocular toxicity observed in chloroquine treatment is associated with disruption of the blood-retinal barrier (BRB). Serious adverse effect associated with long-term use of chloroquine, either as prophylaxis or treatment of rheumatoid arthritis, is irreversible retinopathy $(0.3-2 \%$ incidence). Pruritus especially of the palms and soles occurs frequently in Africans. Rare adverse reactions are photosensitization, aplastic anemia, agranulocytosis, hepatitis, elevated liver enzymes, skin eruptions, psychiatric disturbances, peripheral neuropathy, myopathy, and effect on neuromuscular transmission (muscle weakness). There is some evidence that the drug may induce an autoimmune disorder particularly with long-term high-dose therapy. Several patients with autoimmune diseases (rheumatoid arthritis and SLE) developed clinical, physiological, and pharmacological evidence for myasthenia gravis after prolonged use of chloroquine. Chloroquine may affect kidney function when taken either during treatment or prophylaxis of malaria through accumulation in adrenal gland and the epithelial cells of the kidney. Chronic administration of chloroquine has been reported to cause $\mathrm{Na}+$ retention possibly via increase in plasma aldosterone concentrations and renal $\mathrm{Na}+-\mathrm{K}+-$-ATPase activity. The $\mathrm{S}(-)$ chloroquine is more toxic in humans than $\mathrm{R}(+)$ chloroquine.

Transiently high plasma chloroquine concentrations after the rapid intravenous injection or large intramuscular doses of chloroquine (previously used for treatment of chloroquine-sensitive severe P. falciparum malaria) are associated with cardiovascular toxicity. Toxic manifestations appear rapidly within 1-3 $\mathrm{h}$ after injection and include circulatory arrest, shock, cardiac conduction 
disturbances, and ventricular arrhythmia. Abnormalities in the electrocardiogram (ECG), i.e., QRS complex widening, flattening of the QRS, and ST segment depression, have been reported after overdoses as well as in patients after therapeutic doses. Respiratory depression can also occur.

\section{Contraindications}

Chloroquine is contraindicated in individuals with known hypersensitivity to chloroquine or structurally related aminoquinoline antimalarials.

\section{Caution}

Chloroquine may exacerbate the severity of psoriasis, neurological (e.g., epilepsy) and gastrointestinal disorders, and retinal, visual, or hepatic impairment. The drug should be administered in patients with these underlying conditions with caution.

\section{Pharmacokinetics}

Pharmacokinetic parameters of chloroquine and its active plasma metabolite monodesethylchloroquine following currently recommended doses for malaria treatment and prophylaxis are summarized in Table 1. Chloroquine is rapidly and almost completely absorbed from the gastrointestinal tract after oral administration in either healthy subjects or children with malaria. The oral bioavailability is almost complete, i.e., approximately $90 \%$. Absorption is relatively unaffected by concomitant ingestion of food. However, intersubject variability of $30-100 \%$ has been reported in extent of absorption, which may explain in part the individual variability of chloroquine effectiveness and toxicity. In one study following a single oral dose of $300 \mathrm{mg}$ chloroquine base, peak plasma chloroquine levels of 56-102 ng/ml were attained within 1-6 h. In another study where $10 \mathrm{mg}$ chloroquine per $\mathrm{kg}$ body bw was administered in the form of tablets to children with malaria, a $\mathrm{C}_{\max }$ of about $250 \mathrm{ng} / \mathrm{ml}$ was reached in $2 \mathrm{~h}$ with an absorption half-life of $0.56 \mathrm{~h}$. Multiple doses of $250 \mathrm{mg}$ daily lead to stable plasma concentrations of $100-500 \mathrm{ng} / \mathrm{ml}$.

The pharmacokinetics of chloroquine is complex with plasma levels determined by the rate of distribution rather than by the rate of elimination.
In systemic circulation, about $50-70 \%$ of chloroquine is bound to plasma protein, mainly to $\alpha_{1}$-acid glycoprotein. $\mathrm{S}(+)$ Chloroquine binds more to albumin $(50 \%)$ than $\mathrm{R}(-)$ chloroquine $(35 \%)$. On the other hand, $\mathrm{R}(-)$ chloroquine binds more to $\alpha_{1}$-acid glycoprotein $(48 \%)$ than $\mathrm{S}$ $(+)$ chloroquine( $35 \%)$. Chloroquine is extensively distributed throughout the body. The drug is deposited in tissues (liver, spleen, kidney, lung, retina, and skin) with about 200-20,000 times the plasma concentration. Highest concentrations of chloroquine are found in melanin-containing cells in the retina and skin. The drug remains in the skin for 6-7 months after cessation of therapy at a time when the drug is no longer detectable in the plasma. The extent of accumulation of the $R(-)$ chloroquine in ocular tissues is greater than that of the $\mathrm{S}(+)$ enantiomer. Chloroquine readily crosses the placenta and is excreted in small amount into the breast milk. The milk-to-plasma ratio ranges from 1.96 to 4.26. Cord blood concentration is similar to maternal blood level. Correlation is observed between saliva and plasma levels of chloroquine with saliva: plasma concentration ratio of 0.53 . Due to its extensive tissue distribution $(\mathrm{Vc} / \mathrm{F})$, the apparent volume of distribution $(\mathrm{Vd} / \mathrm{F})$ is extremely large $(31-2621 / \mathrm{kg})$, but the volume of the central compartment is relatively small. This results in transiently high plasma chloroquine concentrations after parenteral administration with wide peak-to-trough fluctuations.

The distribution of chloroquine within the human blood is also important since the malaria parasite is intraerythrocytic during schizogony. Chloroquine preferentially concentrates in blood components, e.g., erythrocytes, platelets, and granulocytes. Erythrocytic concentration is about two to five times of the concurrent plasma concentrations. Concentration in parasitized erythrocytes is about 25 times of normal erythrocytes. Concentration in the whole blood is highest, followed by serum and plasma. It is thought that chloroquine-induced redistribution of a neutral aminopeptidase may be the cause of hemoglobin accumulation in endocytic vesicles of malaria parasites.

Clearance of chloroquine is mainly by renal excretion (40-70\%) and hepatic metabolism 


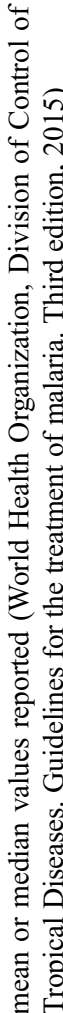

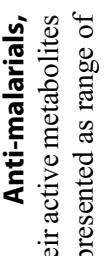

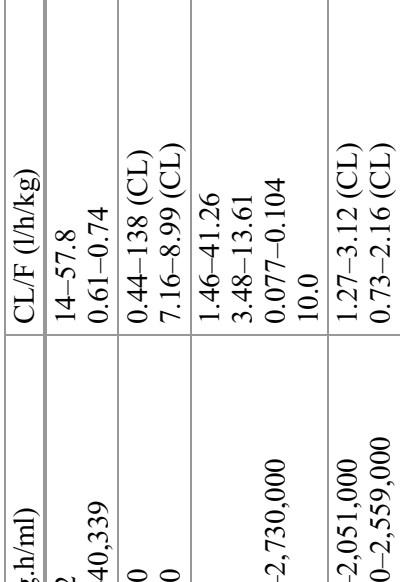

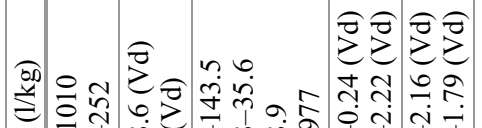

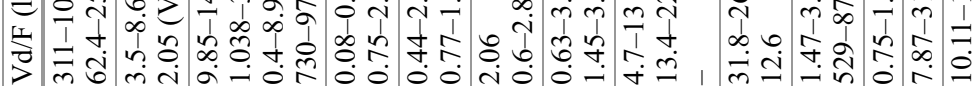

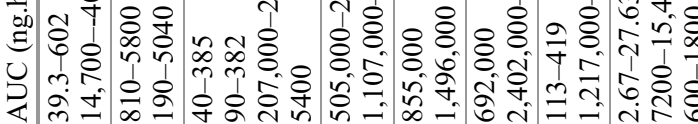

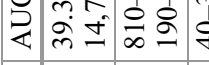

ق

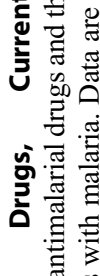

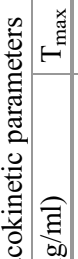

ă $\infty$

1 I I 1 a

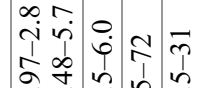

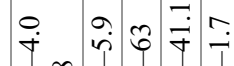

已્తి

$\stackrel{\partial}{>}$

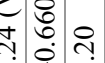

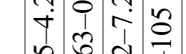

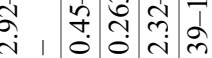

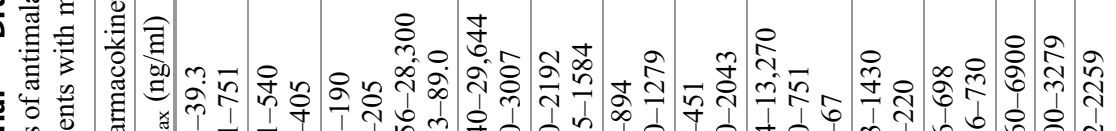

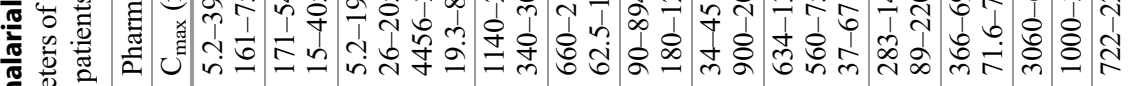

旅 要

능

诺

० : हn

总

ते है छ

응 젼.

हूँ

.

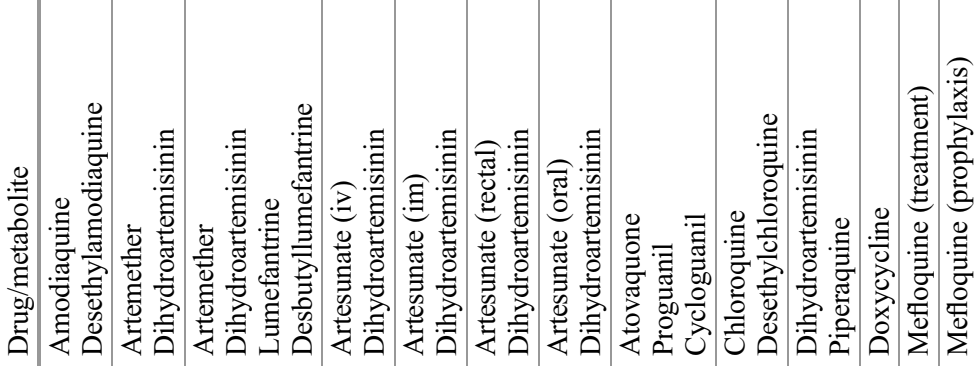

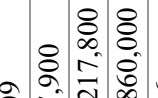

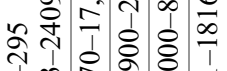

尚管会命客 
(35-50\%). Hepatic metabolism is largely by side chain de-ethylation, leading first to monodesethylchloroquine $(30-40 \%)$ and then to didesethychloroquine or bisdesethylchloroquine (5-10). Monodesethylchloroquine (Fig. 1b) is the main metabolite of chloroquine with similar antimalarial activity against chloroquinesusceptible $P$. falciparum as the parent compound. Bisdesethylchloroquine is further deaminated to form an alcohol (the 4'-hydroxy compound) which is oxidized to the 4'-carboxylic acid derivative. Successive dealkylation of the side chain ultimately produces the compounds 7-chloro-4aminoquinoquinoline, chloroquine side chain $\mathrm{N}$-oxide, and chloroquine di- $\mathrm{N}$-oxide. Metabolism of chloroquine involves the cytochrome P450 (CYP450) system, i.e., CYP2C8, CYP3A4/5, and, to a much lesser extent, CYP2D6, although their quantitative in vivo contribution remains unclear. All the metabolites are toxicologically important. Metabolism of chloroquine occurs slowly, and the main metabolite varies in different species. Extrahepatic sites of microsomal metabolism could also be of clinical significance in view of the extensive tissue distribution of chloroquine and the extrahepatic distribution of CYP3A isoenzymes.

The monodesethylchloroquine metabolite has similar profiles of distribution and tissue binding as the parent drug and can be detected in plasma $30 \mathrm{~min}$ after drug administration. Time to maximum blood concentration $\left(t_{\max }\right)$ of $2-7 \mathrm{~h}$ after the oral dose occurs at approximately the same time as that of chloroquine. The binding of monodesethylchloroquine to $\alpha_{1}$-acid glycoprotein is higher than that to albumin $(21 \%$ vs $3 \%)$. The concentration of monodesethylchloroquine remains at a value of $25-40 \%$ of that of the parent compound after the peak has been reached. At steady state, the ratio of chloroquine to desethylchloroquine is 6-10\%. Bisdesethylchloroquine concentration reaches $10-13 \%$ of chloroquine concentration.

Chloroquine exhibits a multiexponential elimination pattern with a more rapid initial elimination phase, followed by a slower phase. The systemic clearance of chloroquine $(\mathrm{CL} / \mathrm{F})$ varies between 0.23 and $0.80 \mathrm{l} / \mathrm{h} / \mathrm{kg}$. Renal clearance accounts for up to $70 \%$ of the total systemic clearance by both glomerular filtration and tubular secretion, in the forms of unchanged (about 50\%) or metabolized drugs $(25 \%)$. The excretion is increased by acidification of the urine. The $\mathrm{S}(+)$ enantiomer is excreted by the kidneys preferentially compared to the $\mathrm{R}(-)$ enantiomer. Approximately $8-25 \%$ and $5 \%$ are excreted in unchanged form or metabolites in the feces and skin, respectively. About $25-45 \%$ is stored long term in lean body tissues. The reported values of $t_{1 / 2}$ of chloroquine and metabolite vary considerably, depending largely on the duration of sampling and the analytical methods used, i.e., 2.5 and 10-60 days for 7 days and $>56$ days sampling, respectively. The elimination half-lives $\left(t_{1 / 2}\right)$ of chloroquine, mondesethylchloroquine, and bisdesethylchloroquine range from 20 to 60 days. The $t_{1 / 2}$ of the modesethyl metabolite is longer than those of the parent compounds. Chloroquine and metabolites can be found in urine for months after a single oral dose.

\section{Factors Associated with Altered Drug Exposure} and/or Treatment Response

Various pharmacokinetic studies suggest noticeable interindividual variability in chloroquine and monodesethylchloroquine concentrations, and this variability may influence the parasitological treatment outcome. In most cases, blood/plasma concentrations in patients with treatment failure tended to be lower than those with sensitive treatment outcome. In addition, this variability may also associated with increased chloroquine toxicity particularly retinopathy.

Malaria Infection There appears to be no pharmacokinetic difference of chloroquine, besides the higher $\mathrm{C}_{\max }$ found in Thai patients with malaria compared with healthy subjects following an intravenous infusion of chloroquine diphosphate (15 $\mathrm{mg}$ base $/ \mathrm{kg} \mathrm{bw})$. The binding of chloroquine to plasma protein is not altered in malaria infection.

Pregnancy The $\mathrm{CL} / \mathrm{F}$ and $\mathrm{Vd} / \mathrm{F}$ of chloroquine may be increased, while area under blood concentration-time curve (AUC) decreased in pregnant patients. Decreases of $25 \%$ and $45 \%$ in 
the AUCs of chloroquine and desethylchloroquine, respectively, suggest lower exposure that may compromise therapeutic efficacy. Recommendation to increase chloroquine daily dose to four tablets $(1,000 \mathrm{mg})$ regimen in pregnant women is proposed based on results of a population-based pharmacokinetic study to improve clinical efficacy of chloroquine. Clinical studies evaluating safety and potential harm to the fetus are needed. Data on the use of antimalarials by lactating mothers have been limited.

Children and Elderly Information on the influence of age on the pharmacokinetics of chloroquine has been limited. It has only been recognized recently that the currently recommended on the mg per kg bw doses of chloroquine achieve substantially lower plasma drug concentrations in young children than in older patients.

Renal Diseases Kidney or liver dysfunction decreases excretion of chloroquine and leads to greater drug retention and higher risk of chloroquine toxicity. In patients with renal insufficiency, chloroquine elimination is reduced resulting in the prolongation of the $t_{1 / 2}$ of the drug. Blood concentrations at equilibrium level of chloroquine are about $70 \%$ higher in patients with renal impairment. However, this pharmacokinetic alteration is of therapeutic relevance only when chloroquine is used for prophylaxis but not for treatment, as the concentration-time profiles of chloroquine are largely governed by distribution phase rather than elimination phase during the first 3-4 days acute phase of infection.

Malnutrition In malnourished patients, the metabolism of chloroquine is impaired and may result in high blood concentrations.

Ethnics In general, the pharmacokinetics of chloroquine does not differ substantially in different ethnic groups. However, in one study, the rate of chloroquine excretion of during the first $7 \mathrm{~h}$ of administration was higher in Thais as compared to British, Gambian, and Sudanese subjects. The proportions of excreted monodesethylchloroquine to chloroquine were similar in all groups.

\section{Genetic Polymorphisms in Drug-Metabolizing} Enzymes and Transporters The role of polymorphisms of CYP2C8, the key enzyme in chloroquine biotransformation, as a factor that influences treatment outcome has not yet been investigated. It is, however, unlikely to be clinically relevant since both parent compound and metabolite are pharmacologically active. The contribution of polymorphism in protein transporters particularly regarding chloroquine-induced adverse drug effects of the central nervous system (CNS) remains to be clarified.

Drug Interactions There is evidence from in vitro and in vivo animal studies that chloroquine is markedly antagonistic to some antimalarials such as quinine, mefloquine, amodiaquine, and artemisinin. On the other hand, the combination with sulfadoxine, pyrimethamine, and erythromycin is synergistic. Clinical relevance for the interaction of chloroquine with some of these drugs has been demonstrated. In clinical situation where concomitant therapy of chloroquine with other antimalarials cannot be avoided, monitoring of chloroquine blood concentrations is suggested. Despite the encouraging results for the synergistic effects of the combination of chloroquine and erythromycin in vitro and in vivo, the combination of chloroquine and erythromycin does not prove effective in the treatment of highly chloroquineresistant $P$. falciparum in Thailand or even less chloroquine-resistant parasites in Kenya. Desferrioxamine, the only iron chelator used in human medicine, has been shown to inhibit P. falciparum growth both in vitro and in an animal model. However, marked antagonism is observed between desferrioxamine and chloroquine.

Calcium channel blockers, tricyclic antidepressants, and antihistamines inhibit chloroquine transport by P-glycoprotein resulting in increased blood chloroquine levels and antimalarial activity. The antihistamine cyproheptadine has been shown to reverse resistance to chloroquine in $P$. falciparum both in vivo and in vitro.

Food appears to enhance the extent of chloroquine absorption. The AUC and $\mathrm{C}_{\max }$ of chloroquine are significantly elevated when chloroquine 
was administered with rice-based meals, although the rate of absorption is not affected.

In animal studies, acetylsalicylic acid delayed the absorption of chloroquine, but distribution, plasma protein binding, and elimination were not affected. Clinical relevance of these findings remains to be established.

Concomitant therapy of chloroquine with some antacids or antidiarrheal agents results in poor bioavailability of chloroquine.

Activated dimethicone does not appreciably affect the absorption of chloroquine, but calcium carbonate, kaolin, and magnesium trisilicate significantly decrease the absorption of chloroquine.

Activated charcoal has been shown to drastically reduce the absorption of chloroquine. The plasma AUC and $\mathrm{C}_{\max }$ of chloroquine were reduced by $99 \%$ in the presence of activated charcoal. Activated charcoal should have a role in reducing the absorption in chloroquine intoxication.

The co-administration of primaquine with chloroquine for radical treatment of $P$. vivax malaria does not affect chloroquine or monodesethylchloroquine pharmacokinetics but significantly increases plasma primaquine concentrations. This pharmacokinetic interaction may explain previous observations of synergy in preventing $P$. vivax relapse. Similarly, nonclinically significant safety or pharmacokinetic/ pharmacodynamic interactions are found with the co-administration of tafenoquine (antirelapse drug alternative to primaquine) and chloroquine in healthy subjects.

Cimetidine, but not ranitidine, impairs the elimination of chloroquine through inhibition of drug-metabolizing enzymes. Ranitidine is, therefore, the $\mathrm{H}_{2}$-receptor antagonist of choice for ulcer patients receiving chloroquine therapy. Plasma concentration of chloroquine is also increased when co-administered with paracetamol.

Alkalinization of urine decreases chloroquine excretion. Acidification of the urine by oral ingestion of ammonium chloride can increase renal excretion of chloroquine by $20-80 \%$. Intramuscular injection of dimercaprol can also increase urinary excretion of chloroquine.

Chloroquine has been shown to reduce bioavailability of ampicillin, praziquantel, and methotrexate, as well as reduce therapeutic effect of thyroxine. On the other hand, the drug increases plasma concentration of cyclosporine.

Concurrent administration of chloroquine and ethanol has been shown to induce extensive damage to the proximal tubules and collective duct cells of the kidney, probably due to modulatory effect of chloroquine on the renal tubular response to vasopressin, either directly by inhibiting cyclic AMP generation or indirectly via induction of nitric oxide production.

\section{Amodiaquine}

Amodiaquine is an analog of chloroquine, which is effective against low-level chloroquineresistant $P$. falciparum malaria. In the 1980 s, the use of amodiaquine was discouraged due to rare but serious idiosyncratic hepatotoxicity and agranulocytosis after long-term prophylaxis. In the 1990s, its use was reconsidered following the widespread development of chloroquine resistance. It is now recommended by WHO to be used in combination with artesunate as one of the artemisinin-based combination therapies (ACTs) for the treatment of uncomplicated $P$. falciparum and $P$. vivax in areas where the parasites are sensitive to amodiaquine. Recently, WHO has recommended seasonal malaria chemoprevention (SMC) using a complete treatment of sulfadoxine-pyrimethamine and amodiaquine once a month for 4 months during the malaria transmission season for children aged between 3 and 59 months.

\section{Chemistry and Physical Properties}

Amodiaquine (4-[(7-Chloroquinolin-4-yl)amino]2-[(diethylamino)methyl]phenol: Fig. 2a) is a Mannich base 4-aminoquinoline that is similar in chemical structure and mechanism of action to chloroquine. Amodiaquine is a yellow crystalline powder, odorless (or almost odorless) with a bitter taste. Soluble 1 in 22 parts water 1 in 70 parts ethanol (96\%). The molecular weight is 355.9 . Amodiaquine is practically insoluble in benzene, chloroform, and ether. It is decomposed at temperature $150-160{ }^{\circ} \mathrm{C}$. The drug is used as a racemic mixture of equal amounts of $\mathrm{S}(+)$ and $\mathrm{R}(-)$ amodiaquine. 

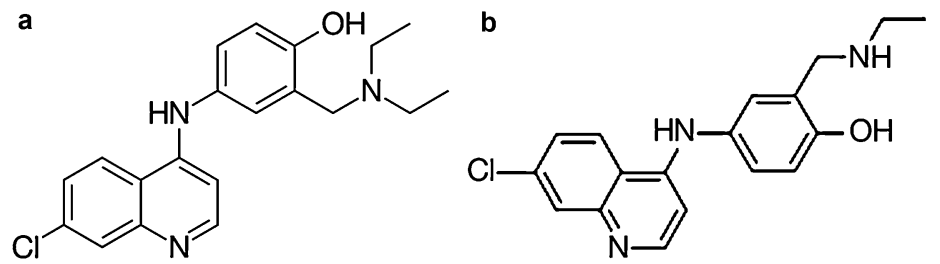

Pharmacology of Antimalarial Drugs, Current Anti-malarials, Fig. 2 Chemical structures of (a) amodiaquine and (b) monodesethylamodiaquine

Pharmacological Activities

Antimalarial Activity and Mechanism of Action and Resistance Amodiaquine has antimalarial activity on various Plasmodium stages similarly to that of chloroquine. It is active against asexual erythrocytic forms of $P$. vivax, $P$. ovale, $P$. malariae, $P$. knowlesi, and chloroquinesensitive $P$. falciparum. It is also active against gametocytes of $P$. vivax, P. malariae, $P$. ovale, and immature forms of $P$. falciparum. The drug has no activity against the exoerythrocytic tissue stages of malaria. Its major plasma metabolite monodesethylamodiaquine (Fig. 2b) has similar antimalarial activity. The mechanisms of action and resistance of amodiaquine have not been fully elucidated but are thought to be similar to chloroquine.

\section{Therapeutic Indications for Malaria}

Oral amodiaquine is currently available as a single tablet and a fixed-dose combination (ACT) in tablets containing $25 / 67.5 \mathrm{mg}, 50 / 135 \mathrm{mg}$, or $100 / 270 \mathrm{mg}$ of artesunate-amodiaquine. Amodiaquine in combination with artesunate as one of the ACTs is indicated for the treatment of uncomplicated $P$. falciparum and $P$. vivax malaria. The combination is also active against $P$. vivax, $P$. ovale, P. knowlesi, and P. malariae. The recommended dose regimen for ACT is a total therapeutic dose range of $6-30 \mathrm{mg} / \mathrm{kg}$ bw per day artesunate and $22.5-45 \mathrm{mg} / \mathrm{kg}$ bw per day amodiaquine for 3 days. The combination may also be used as follow-on treatment in patients with severe malaria if oral medication is tolerated. In areas with high malaria transmission in the subSahel region of Africa where the majority $(>60 \%)$ of clinical malaria cases occur during a short period ( $\leq 4$ months), seasonal malaria chemoprevention (SMC) with monthly amodiaquine in combination with sulfadoxine-pyrimethamine is recommended for all children aged less than 6 years during each transmission season. The combination is given at full treatment doses, i.e., a single dose of sulfadoxine-pyrimethamine $(25 / 12.5 \mathrm{mg})$ and 3 days of amodiaquine $(10 \mathrm{mg} / \mathrm{kg}$ bw per day) at monthly intervals.

\section{Adverse Reactions and Toxicity}

The adverse reactions of amodiaquine are generally mild to moderate and are similar to chloroquine. Hepatic problems or decrease in blood counts may rarely occur. Toxicity from overdose may include headache, seizures, and cardiac arrest.

Artesunate-amodiaquine is generally welltolerated. Common adverse reactions include gastrointestinal disturbances (nausea and abdominal pain), cough, anorexia, insomnia, fatigue, and weakness. The incidence of gastrointestinal disturbances is higher compared with other ACTs. Less common adverse reactions are arrhythmia, bradycardia, vomiting, extrapyramidal effects, retinopathy, and pruritus. Eye disorders (transient accommodation disorders and corneal opacification) varying in types and severity have also been reported but regress upon termination of treatment. Serious adverse reactions are neutropenia and hepatotoxicity. These serious effects are most often with prolonged use of amodiaquine for prophylaxis or prolonged use of artesunateamodiaquine treatment.

Amodiaquine-SP is generally well tolerated in children. Common adverse reactions include vomiting, loss of appetite, fever, and mild-to- 
moderate skin reactions. No serious adverse reactions have been reported.

\section{Contraindications}

Amodiaquine should not be administered to patients with known hypersensitivity to amodiaquine and structurally related drugs. In addition, clinical use with this drug should be avoided in patients with history of hepatotoxicity, hepatic impairment, neutropenia, or retinopathy.

\section{Cautions}

Although there is no evidence for life-threatening cardiovascular complications of amodiaquine as what has been reported with overdose of chloroquine, caution should be made in treating patients who have recently taken another antimalarial drug with cardiovascular adverse reactions such as quinine, mefloquine, or even chloroquine.

\section{Pharmacokinetics}

Pharmacokinetic parameters of amodiaquine and its active plasma metabolite following currently recommended doses for treatment of uncomplicated malaria and seasonal malaria chemoprevention are summarized in Table 1.

After oral administration, amodiaquine hydrochloride is rapidly absorbed and undergoes rapid and extensive metabolism by CYP2C8 to the active metabolite monodesethylamodiaquine (Fig. 2b) and the secondary metabolite 2-hydroxyamodiaquine which are eliminated by renal excretion. Monodesethylamodiaquine is concentrated in red blood cells; whole blood to plasma ratio is approximately $3: 1$. Both the parent drug and metabolite are highly ( $>90 \%$ ) plasma protein bound. It is likely that the distribution of monodesethylamodiaquine in man mirrors that of chloroquine, i.e., wide distribution into the body tissues, particularly in the liver, spleen, kidney, lungs, brain, and spinal cord. It also binds to melanin-containing cells in the eyes and skin. Although amodiaquine is three times more potent than monodesethylamodiaquine, monodesethylamodiaquine remains longer in blood where it concentrates in red blood cells and is claimed responsible for the most of the antimalarial efficacy of amodiaquine. Blood concentrations of amodiaquine and monodesethylamodiaquine show wide (more than ten times) variation between individuals.

\section{Factors Associated with Altered Drug Exposure} and/or Treatment Response

Malaria Infection The oral pharmacokinetic profiles of amodiaquine and monodesethylamodiaquine obtained from patients with malaria and healthy subjects are similar, except the absorption which is significantly delayed in patients from a mean of $0.5-1.75 \mathrm{~h}$.

Children Small studies did not find any effect of age on plasma concentrations of monodesethylamodiaquine or amodiaquine itself. However, it was reported that treatment failure after amodiaquine monotherapy was more frequent among children who were underweight for their age.

Pregnancy There are no data published on the pharmacokinetics of amodiaquine in pregnant women although it was previously used to treat malaria in pregnancy.

HIV Coinfection Artesunate-amodiaquine combination is associated with severe neutropenia, particularly in patients coinfected with HIV and especially in those on zidovudine and/or cotrimoxazole treatment. Concomitant use of efavirenz increases exposure to amodiaquine and hepatotoxicity. Concomitant use of this ACT with these drugs should therefore be avoided.

\section{Genetic Polymorphisms in Drug-Metabolizing}

Enzymes Enzymatic and nonenzymatic formation of highly reactive amodiaquinequinoneimine, a protein-arylating intermediate, is thought to be responsible for serious organ-damaging amodiaquine adverse reactions observed in some patients. The effects of CYP2C8 polymorphisms on amodiaquine efficacy and toxicity remain conflicting. Theoretically, a slower conversion of amodiaquine to monodesethylamodiaquine in the CYP2C8 poor metabolizers might predispose patients to form this highly reactive intermediates 
and, thus, increased risk of toxicity. The pharmacological activity of both amodiaquine and monodesethylamodiaquine, their antiparasitic synergism, or their extrahepatic metabolism might have hidden a CYP2C8 poor metabolizer phenotype and prevented treatment failure.

Drug Interactions Amodiaquine has potential to inhibit CYP2D6 and CYP2C9 activities. On the other hand, amodiaquine metabolism may be inhibited by CYP2C8 and CYP2A6 inhibitors. Plasma concentration of chloroquine including CYP450 activities has been shown to be increased when co-administered with the anti-HIV efavirenz. On the other hand, the concentration is decreased when co-administered with nevirapine.

With regard to pharmacodynamic interactions, increased risk for cardiotoxic effects may occur if amodiaquine is co-administered with antiarrhythmic drugs. In addition, the risk for neutropenia may be increased when amodiaquine is co-administered with zidovudine-containing regimens and trimethoprim-sulfamethoxazole combination.

\section{Primaquine}

Primaquine was first synthesized as the most potent compound in a large series of quinoline derivatives in 1946, by Elderfield and colleagues at Columbia University, and was later on investigated in humans as part of the US government's Malaria Project conducted at the Stateville Penitentiary. The first mass use of primaquine was in the Korean War in over 250,000 US soldiers as a tissue schizonticide to eliminate long latency $P$. vivax infections with a 14-day primaquine course. Since then, primaquine has gradually become the standard therapy in treating relapsing malaria and an important tool in malaria transmission blocking (gametocytocidal activity) and elimination campaigns with high effectiveness. Its effectiveness has nevertheless always been challenged with availability, prescribing practices, and patients' compliance. Most importantly, hemolytic toxicity particularly in individuals with glucose-6-phosphate dehydrogenase (G6PD) deficiency is of concern, which significantly decreases effectiveness of primaquine.

\section{Chemistry and Physical Properties}

Primaquine [8-(4-Amino-1-methylbutylamino)-6methoxy-quinoline: Fig. 3a] is an 8 -aminoquinoline antimalarial. Primaquine is a racemate with equal parts of $\mathrm{S}(+)$ and $\mathrm{R}(-)$ forms because of the presence of an asymmetric carbon atom. The molecular weights of the base and diphosphate salts are 259 and 455 . The diphosphate is the commercially available salt. It is soluble in water, and its solutions are stable, although some decomposition may take place on exposure to light and air.

\section{Pharmacological Activities}

Antimalarial Activity Primaquine is highly active against exoerythrocytic forms (hypnozoites) and sexual stages (gametocytes) of all Plasmodium species. It has weak activity against asexual blood stages of $P$. vivax but has negligible activity against P. falciparum.

Primaquine is active against primary tissue stages in the liver of all human Plasmodium species and can be considered to be a causal prophylactic agent. However, due to its toxicity, it has not been used for this purpose on a large scale. It is the only compound available for clinical use in the treatment of hypnozoite tissue stages form of $P$. vivax and $P$. ovale, to prevent relapses of these two species. It is also the only active drug against mature gametocytes of $P$. falciparum and is used routinely in areas under active control of malaria transmission. Primaquine has no substantial effect on blood stage of human malaria unless toxic concentrations are achieved.

The exact mechanism of action of primaquine as anti-hypnozoite and gametocytocide remains unclear. The main mechanism is proposed to be through parasite metabolism in mitochondria, eventually by interference with the ubiquinone function as an electron carrier in the respiratory chain. Another potential mechanism is the production of highly reactive metabolites that generate intracellular oxidative potentials. Primaquine has been shown to bind to PfCRT and can thereby inhibit chloroquine transport. This may explain the synergistic action between the two animalarials as well as the reversal of chloroquine resistance. Biotransformation of primaquine 
appears to be necessary for their toxicity as well as efficacy. Selective generation of oxidative stress in the parasitized cells is the most plausible mechanism for primaquine toxicity and efficacy. It is possible that primaquine may have two entirely different modes of action, one through the parent compound and another through its metabolite(s). Different metabolites exhibit a varying degree of antimalarial activity. However, it is still unclear whether these metabolites or the parent compound exert the most important antiparasitic action. Current knowledges suggest that the 4-amino-1methyl side chain is important in antimalarial activity. The 5-hydroxydemethylprimaquine (formed by deamination and oxidation of primaquine) is the most active metabolite. Carboxyprimaquine is considerably less active than primaquine against exoerythrocytic stages of $P$. berghei in vitro.

The resistance to primaquine is incredibly low. This may be associated with its physical, chemical, or biological properties, together with its short half-life and ability to sterilize the parasite's gametocytes. Relapses of $P$. vivax malaria shortly after primaquine therapy have been reported in the Western Pacific, Southeast Asia, India, and Central and South America. Nevertheless, the frequency, intensity, and distribution of those isolated reports do not appear alarming. Primaquine resistance is commonly confused as failure of therapy or inability to remove the hypnozoite liver stage of $P$. vivax after the full course of therapy and correct therapeutic dose. The other important factor in detection of therapy failure with primaquine is adherence to medication. The exact mechanism of resistance of primaquine has not been identified.

\section{Other Pharmacological Activities and}

Clinical Uses Primaquine is useful for prophylaxis and treatment of moderate Pneumocystis carinii pneumonia (PCP) or as salvage therapy when given in combination with clindamycin. The drug interferes with the microbial electron transport system by producing quinone metabolites that generate superoxides in vivo. The effective daily dose for PCP treatment is $2 \mathrm{mg} / \mathrm{kg}$ bw of primaquine and $225 \mathrm{mg} / \mathrm{kg}$ bw of clindamycin. Lower doses are applied for prophylaxis.
Primaquine has been shown to exhibit leishmanicidal activity in vitro but has no clinical application against leishmaniasis. Some derivatives of primaquine, particularly 6-desmethyl-8aminoquinolines, have been generally more active in vitro than the parent drug against macrophagecontained leishmania.

Both primaquine and 2-methyl-PQ derivative have been reported to be almost four times as effective as the standard drug nifurtimox, against Chagas model disease in mice. This antitrypanosomiasis activity of primaquine relies on the metabolic formation of free radicals that increase the oxidative stress in T. cruzi. The drug is, however, not clinically used for the therapy of Chagas disease.

Other biochemical and physiological activities of primaquine demonstrated by in vitro or in vivo studies include inhibition of vesicular transport and blocking of the calcium-release-activated current, blocking of the appearance of $\mathrm{Ca}^{2+}$ influx currents in response to $\mathrm{Ca}^{2+}$ store depletion, blocking of $\mathrm{Na}^{+}$and $\mathrm{K}^{+}$cardiac channels, and inhibition of diverse types of voltage-gated ionic channels, as well as acetylcholine $(\mathrm{ACh})$ receptors.

\section{Therapeutic Indications for Malaria}

Primaquine is indicated for malaria on four different clinical uses: (i) radical cure in individuals infected with $P$. vivax or $P$. ovale; (ii) presumptive antirelapse therapy (terminal prophylaxis) for persons extensively exposed to $P$. vivax or P. ovale; (iii) reduction of onward transmission of P. falciparum malaria in programs to eliminate P. falciparum malaria and in areas threatened by resistance of $P$. falciparum to artemisinins; and (iv) primary prophylaxis against all species of malaria. For radical cure of $P$. vivax and $P$. ovale infections, the efficacy is highly dependent on the concurrent administration of an effective blood schizontocidal agent. Primaquine is not recommended for use as a single drug as it is not effective against erythrocytic forms of Plasmodia; the drug must be co-administered with blood schizonticides. Primaquine dosage is customarily expressed in terms of the base. Primaquine phosphate USP is supplied in tablets containing 7.5 and $15 \mathrm{mg}$ of base. 
Pharmacology of Antimalarial Drugs, Current Anti-malarials,

Fig. 3 Chemical structures of (a) primaquine and

(b) carboxyprimaquine
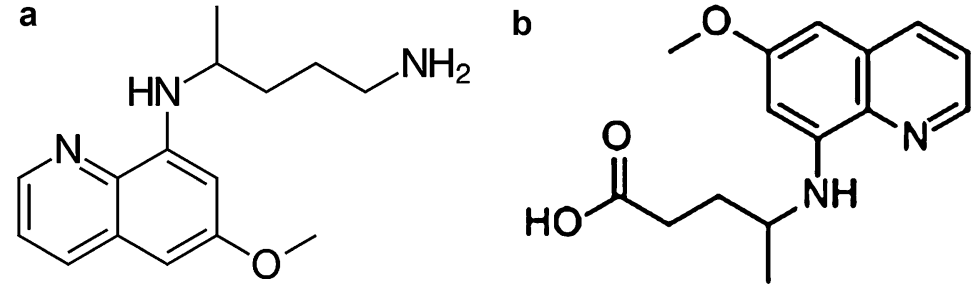

A 14-day course $(0.25-0.5 \mathrm{mg} / \mathrm{kg}$ bw daily) of primaquine with blood schizonticide (chloroquine or an ACT) is strongly recommended in children and adult patients (except pregnant women, infants aged lower than 6 months, women breast-feeding infants aged less than 6 months, women breast-feeding older infants unless they are known not to be G6PD deficient, and patients with G6PD deficiency) with $P$. vivax or $P$. ovale in all transmission settings (except pregnant women, infants aged less than 6 months, and women breast-feeding infants aged less than 6 months) to prevent relapse. In patients with mild-tomoderate G6PD deficiency, primaquine base is given at $0.75 \mathrm{mg} / \mathrm{kg}$ bw once a week for 8 weeks, with close medical supervision during the first 3 weeks of treatment for potential primaquine-induced hemolysis. In patients with severe G6PD deficiency, risk-benefit assessment needs to be taken on an individual basis by experienced tropical medicine physicians. When G6PD status is not known and G6PD testing is not available, a decision to prescribe primaquine must be based on an assessment for the risks and benefits of adding primaquine. In pregnant women, primaquine should be given on the basis of G6PD status to prevent future relapse following chloroquine weekly prophylaxis (until delivery and breast-feeding are completed).

A single dose of $0.25 \mathrm{mg} / \mathrm{kg}$ bw primaquine with an ACT is currently recommended in patients with uncomplicated malaria in low-transmission areas (except pregnant women, infants aged less than 6 months, and women breast-feeding infants aged less than 6 months) to reduce diseases transmission without G6PD testing. The change in policy from the previously recommended dose of $0.75 \mathrm{mg} / \mathrm{kg}$ bw in 2010 to $0.25 \mathrm{mg} / \mathrm{kg}$ bw in 2015 was based partly on a WHO Evidence
Review Group report summarizing published literatures. This recommendation is later on supported by evidence assessed from a systematic review which suggests that the lower single dose $(0.1-0.25 \mathrm{mg} / \mathrm{kgbw})$ of primaquine given with the goal of reducing transmission of $P$. falciparum is less likely to cause hemolytic effects in people with G6PD deficiency than the previous $0.75 \mathrm{mg} / \mathrm{kg}$ bw dose and that severe hemolytic events are not very common.

\section{Adverse Reactions and Toxicity}

Primaquine is generally well tolerated when the drug is administered in the usual therapeutic doses with minimal adverse reactions. Primaquine biotransformation plays an important role both its toxicity and antimalarial action and involves chiral recognition, even though exact mechanisms are not yet established.

The common adverse reactions include doserelated gastrointestinal discomfort (nausea, vomiting, diarrhea, and abdominal cramps) and dizziness. Abdominal distress from primaquine can be alleviated by antacids and by administering the drug with a meal. The less well-documented adverse reactions of primaquine are derangement of leukocyte levels, i.e., dose-dependent granulocytopenia or agranulocytosis, and effects on immune mechanisms. Other rare adverse effects include hypertension, arrhythmias and corrected QT (QTc) interval prolongation, and symptoms related to the central nervous system. The effects on human fetus are unknown, but the use of primaquine in pregnancy is not recommended due to hemolytic effects. Primaquine has been shown to be mutagenic in Escherichia coli and Salmonella typhimurium test systems.

The two major serious adverse effects of primaquine are the propensity to induce the 
formation of methemoglobin and hemolytic episodes when erythrocytes with deficiency in G6PD are present. The severity of primaquine-induced hematoxicity is related to dose and degree of G6PD deficiency. Methemoglobinemia, a predictable dose-related adverse effect, occurs occasionally with therapeutic dosage but is much more common with a higher than therapeutic dosage. Several of primaquine metabolites, except carboxyprimaquine, have shown greater propensity to cause methemoglobin formation than primaquine itself. Acute intravascular hemolysis is by far the most serious toxic hazard of primaquine which restricts the wider use of the drug. It occurs as a consequence of oxidant stress in individuals with G6PD deficiency, an inherited $\mathrm{X}$ chromosome-linked trait. Primaquine-induced hemolysis is characterized by severe anemia, intravascular hemolysis with dark urine, and mild jaundice. Hemolysis appears usually on the second or third day of administration of primaquine and continues for 5-7 days. The hematocrit level drops to $30 \%$ between days 8 and 12 when about half of the red cells have been destroyed. Hemoglobinuria often accompanies the hemolysis; serum bilirubin rises to 50-90 $\mu \mathrm{M}$, and slight jaundice may appear; Heinz bodies occur commonly at first but disappear as the hemolysis intensifies. The symptoms are related to the effect of the anemia. The hemolysis is normally self-limiting upon withdrawal of the drug. The degree of G6PD deficiency varies greatly ranging from moderate in Africans (African variant A) to very high in people of Mediterranean origin (Mediterranean variant B) and in population groups scattered throughout Asia (Asian variants). Several phenolic analogs of primaquine have been proposed to cause hemolytic effect, e.g., 5-hydroxyprimaquine, demethylprimaquine, and 5-hydroxydemethylprimaquine. It is still uncertain whether the major metabolite carboxyprimaquine contributes significantly to the toxicity of primaquine, although it does not cause methemoglobin formation in vitro. Carboxyprimaquine does not inhibit drug metabolism in vitro; however, inhibition of the formation of carboxyprimaquine could lead to serious adverse effects, i.e., hemolytic anemia or other unexpected toxicity due to the shift of the metabolic pathway to hydroxylated metabolites or other metabolites responsible for this effect.

\section{Contraindications}

Primaquine is contraindicated in patients with known hypersensitivity to primaquine and related compounds as well as in patients with severe G6PD deficiency or methemoglobinemia. Since primaquine crosses the placenta and may cause hemolysis in G6PD-deficient fetus, it is not recommended for use during pregnancy or during breast-feeding unless the G6PD status of the infant is known. Nonclinical data from studies conducted in bacteria and in animals treated with primaquine show evidence of gene mutations and chromosomal/DNA damage, teratogenicity, and injury to embryos and developing fetuses when primaquine is administered to pregnant animals.

Primaquine is also contraindicated for children under 4 years of age, and its administration requires a previous test for G6PD status.

\section{Caution}

Primaquine should not be given to subjects with concurrent treatment with potentially hemolytic drug or agents capable of depressing the myeloid elements of the bone marrow.

Different variants of G6PD deficiency are associated with significantly different risks for hemolysis. Decision to prescribe primaquine must be based on an assessment of the risks and benefits of using primaquine in patients with mildto-moderate G6PD deficiency or when the G6PD status is unknown and G6PD testing is not available. If primaquine administration is considered, baseline hematocrit and hemoglobin levels must be investigated before treatment, and close hematological monitoring (e.g., at day 3 and 8) is required. Adequate medical support to manage hemolytic risk should be available. Due to the potential for QTc interval prolongation, electrocardiogram (ECG) should be monitored when primaquine is administered in patients with cardiac disease, long QTc syndrome, a history of ventricular arrhythmias, uncorrected hypokalemia and/or hypomagnesemia, or bradycardia $(<50 \mathrm{bpm})$ and during concomitant administration with QTc interval prolonging agents. 


\section{Pharmacokinetics}

Pharmacokinetic parameters of primaquine and its plasma metabolite carboxyprimaquine following currently recommended doses in healthy subjects and malaria patients are summarized in Table 1 .

Absorption after oral administration is rapid and almost complete with bioavailability of about $96 \%$. $\mathrm{C}_{\max }$ is achieved within $1-4 \mathrm{~h}$ of dosing. Mean absorption half-life $\left(t_{1 / 2 a}\right)$ is $30 \mathrm{~min}$. The concentration-time profiles in whole blood and in plasma are similar. Primaquine is distributed throughout the body. The $\mathrm{Vd} / \mathrm{F}$ is in the range of $3-81 / \mathrm{kg}$. About $75 \%$ of primaquine is bound to proteins. Red cell concentration is relatively high. The drug also crosses the placenta, but it is not extensively found in breast milk.

Primaquine is predominantly cleared by hepatic metabolism with renal elimination accounting for less than $1 \%$ of the administered dose over $24 \mathrm{~h}$. The $\mathrm{CL} / \mathrm{F}$ is approximately $0.3-1.2 \quad 1 / \mathrm{h} / \mathrm{kg}$ bw. Hepatic clearance of primaquine is via two distinct pathways. Monoamine oxidase (MAO-A) biotransforms primaquine to the predominant but inactive metabolite carboxyprimaquine (Fig. 3b). Carboxyprimaquine is slowly eliminated and also further biotransformed via CYP2C19, CYP2D6, and CYP3A4, which generates the reactive intermediates responsible for antimalarial activities and methemoglobinemia and hemolytic toxicity, particularly the phenolic metabolite 5-hydroxyprimaquine. Carboxyprimaquine reaches plasma concentrations more than ten times higher than those of the parent compound (at approximately 4-8 h). It accumulates with daily dosing of primaquine. In animal studies in the isolated perfused rat liver preparation, the formation of the carboxy metabolite of primaquine was enantioselective. Stereoselective metabolism also plays a role in both antimalarial activity and toxicity. Both primaquine and carboxyprimaquine are excreted mainly via the biliary tract and can be found in feces within $24 \mathrm{~h}$ of administration. Primaquine itself is also excreted in urine. $(-) \mathrm{R}$ primaquine is cleared more extensively to carboxyprimaquine than the $(+) S$ enantiomer. The $t_{1 / 2}$ of primaquine and carboxyprimaquine are approximately $3-8$ and 15-17 h, respectively.

The pharmacokinetics of primaquine and carboxyprimaquine after oral administration in man are unaffected by dose size, within the clinically used dosage range. Primaquine does not inhibit or induce its own metabolism in man; multiple-dose studies indicate that primaquine does not accumulate in the plasma over the 14 days period of treatment. No significant concentrations of primaquine are found in the predose plasma samples taken prior to the daily doses of the drug.

\section{Factors Associated with Altered Drug Exposure and/or Treatment Response}

Malaria Infection A single oral dose of $45 \mathrm{mg}$ primaquine is rapidly absorbed in patients with $P$. vivax or $P$. falciparum malaria. Acute P. falciparum infection is associated with a reduction in the CL/F of primaquine, while $t_{1 / 2}$ remained unaffected. This change in the oral clearance is possibly due to an impairment of the drug-metabolizing enzyme system and/or a reduction in the absorption across the gastrointestinal tract. The pharmacokinetics of primaquine appears to be unchanged in patients with $P$. vivax malaria.

G6PD Status The status of G6PD enzyme activity does not influence the pharmacokinetics of primaquine.

Ethnics Ethnic diversity of primaquine pharmacokinetics suggests potential heritability. In Koreans, the $\mathrm{C}_{\max }$ of primaquine has been shown to be about five times higher and the carboxyprimaquine concentration about two to five times lower than in Indian and Thai populations. Different exposure has also been seen between Caucasian and Thai males.

Gender Conflicting results have been reported on the effects of gender on the disposition of primaquine. Some studies reported increased exposure and hence greater adverse reactions in women, while others reported no effect of gender. 
Renal Diseases The pharmacokinetics of a single oral dose of $15 \mathrm{mg}$ primaquine does not appear to be altered in patients with severely impaired renal function and end-stage renal dysfunction.

Genetic Polymorphisms in Drug-Metabolizing Enzymes Genetic polymorphisms that decrease CYP2D6 enzyme activity reduce bioactivation of primaquine and may result in treatment failure. The treatment options for $P$. vivax infection in poor metabolizer are likely limited. Additionally, the use of primaquine as a prophylactic agent in this population would likely be ineffective. On the other hand, extensive metabolizers would likely be successful with primaquine therapy as they have the lowest parent primaquine levels and highest production of reactive metabolites and primaquine. Individuals with ultrarapid metabolizers would be expected to display the most primaquine metabolism through the CYP2D6 pathway. Relationship between CYP2D6 metabolic status and clinical outcome of primaquine therapy remains to be clarified.

Drug Interactions Primaquine drug-drug interactions are likely complex and involve a multitude of pharmacological factors besides hepatic metabolism, including transporter-mediated drug-drug interactions. Primaquine has been shown to interact with $\mathrm{ABC}$ transporters such as P-glycoprotein (MDR1) and multidrug resistance protein 1 (MRP1). The impact that these drug-drug interactions have on primaquine efficacy is however unclear. A number of antimalarials, particularly those with a quinoline structure, i.e., mefloquine, chloroquine, quinine, and in particular primaquine itself, are all well recognized as inhibitors of hepatic CYP450 in vitro, in vivo in animals, and in man. In the chemotherapy of malaria, these antimalarials are usually co-administered either concurrently or sequentially. Metabolic drug interaction between these drugs is therefore a major concern. Results from an in vitro study showed that chloroquine, quinine, artemether, and artesunate did not significantly inhibit the formation of carboxyprimaquine by human liver microsomes. Prior treatment with chloroquine in the clinical situation had no effect on plasma primaquine pharmacokinetics in Thai patients with $P$. vivax malaria. Similarly, there appears to be no pharmacokinetic interaction between primaquine and mefloquine when used at therapeutic doses, despite mefloquine being a strong inhibitor of the formation of carboxymefloquine in vitro. Co-administration of primaquine with quinine did not alter the pharmacokinetics of primaquine but quinine. Production and/or elimination of carboxyprimaquine was reduced as indicated by the lower values of AUC within $24 \mathrm{~h}$ in the presence of quinine. Primaquine plasma levels and exposure have been shown to be increased when primaquine is given with chloroquine, dihydroartemisinin-piperaquine combination, and pyronaridine-artesunate combination therapy. The underlying mechanism is likely due to inhibition of CYP2D6-mediated primaquine metabolism. Some of the blood stage antimalarial agents that could interact with primaquine through CYP450 metabolic pathways also have the potential to interact with primaquine through P-glycoprotein-mediated transport.

Significant pharmacokinetic and pharmacodynamic interactions between primaquine and other drugs have been reported. The metabolic clearance of antipyrine to its three main metabolites, i.e., 3-hydroxymethylantipyrine, 4-hydroxyantipyrine, and norantipyrine, was significantly reduced after a single oral dose of $45 \mathrm{mg}$ primaquine. There was no effect of primaquine on either conjugation (to paracetamol glucuronide and paracetamol sulfate) or oxidation, which supports the safety of using paracetamol as an antipyretic in malaria patients. In vitro studies have shown that primaquine conversion into carboxyprimaquine is inhibited by ketoconazole.

On the pharmacodynamics aspect, artesunate has been shown to reduce the appearance of gametocytemia in adult Thai patients infected with uncomplicated $P$. falciparum malaria, whereas its combination with primaquine resulted in shorter gametocyte clearance time. Furthermore, pentobarbitone sleeping time and zoxazolamine paralysis time were prolonged in a dose-related manner after acute administration of primaquine in the rat. Adverse hematological 


\section{Pharmacology of Antimalarial Drugs, Current Anti-malarials,}

Fig. 4 Chemical structures of (a) quinine and

(b) 3-hydroxyquinine

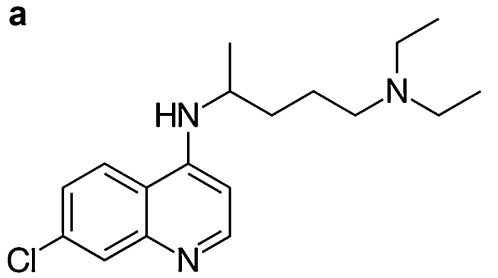

reactions may also occur in the presence of myelosuppressants.

\section{Quinine}

Quinine is the chief alkaloid of the bark of the Cinchona tree (Arbor febrifuga) indigenous to certain regions of South America. It was first used against fever in Peru, probably around 1630. The bark was employed in 1638 to treat the wife of the Viceroy of Peru. Her miraculous cure resulted in the introduction of Cinchona into Spain in 1639 for the treatment of ague. By 1640, the drug was being employed for fever in Europe. Its use was first mentioned in European medical literature in 1643 by a Belgian. The name Cinchona is reported to be a misspelling of Chinchona, the name of the Portuguese countess who allegedly used the compound first.

For almost two centuries, the bark was employed for medicine as a powder, extract, or infusion. It was not until the 1830s that the four main active constituents of Cinchona bark were isolated: two pairs of optical isomers quinine and quinidine, together with cinchonine and cinchonidine. The Cinchona alkaloids have been used ever since, for the treatment of malaria and arrhythmic heart conditions.

\section{Chemistry and Physical Properties}

Cinchona contains a mixture of more than 20 alkaloids. The most important of these are two pairs of optical isomers quinine and quinidine and cinchonine and cinchonidine. Quinidine is a dextrorotatory diastereomer of quinine. Quinine, cinchonine, and cinchonidine are levorotary.

Quinine[6-methoxy- $\alpha$-(5-vinyl-2-quinuclidinyl)4-quinolinemethanol: Fig. 4a] contains a quinoline group attached through a secondary alcohol linkage to a quinuclidine ring. A methoxy side chain is attached to the quinoline ring and a vinyl to the quinuclidine. The molecular weights of base, sulfate, bisulfate, hydrochloride, and dihydrochloride are $324,747,782,361$, and 397, respectively. The salts are freely soluble in water. The drugs should be stored in air-tight containers and be protected from light.

\section{Pharmacological Activities}

Antimalarial Activity and Mechanism of Action and Resistance Quinine is a potent blood schizontocidal (large rings and trophozoites) against all species of Plasmodium and also gametocytocidal against $P$. malariae, $P$. ovale, $P$. vivax, and $P$. knowlesi. Quinine has no tissue schizontocidal or sporontocidal activity. The metabolites exhibit lower antimalarial potency than the parent drug.

The molecular mechanism of action of quinine against $P$. falciparum is only partially understood. Like chloroquine, quinine accumulates in the parasite digestive food vacuole and inhibits the heme detoxification process. Decreasing sensitivity to quinine has also been reported in some areas where the drug has been used extensively for malaria therapy. The genetic basis of quinine resistance involves multiple genes. Similar to chloroquine resistance in P. falciparum, quinine resistance is also associated with mutations of the two transport proteins, PfCRT and PfMDR1. In addition, the transport protein PfNHE1 (Plasmodium falciparum sodium/proton exchanger 1) encoded by the $P$. falciparum $\mathrm{Na}+/ \mathrm{H}+$ exchanger gene $p$ fnhe on chromosome 13 is also involved.

\section{Other Pharmacological Activities and} Clinical Uses Apart from malaria, quinine is also used to treat lupus and arthritis. The drug was previously frequently prescribed as an offlabel treatment for leg cramps at night, but this 
has become less common due to a Food and Drug Administration warning that this practice is associated with life-threatening adverse reactions.

Quinine has oxytocic potential to induce premature labor. It is therefore used to augment labor and also to induce abortion.

\section{Therapeutic Indications for Malaria}

Quinine and its dextroisomer quinidine are one of the most commonly used drugs for malaria treatment worldwide. Quinine is a basic amine and is usually provided as a salt. Various existing preparations include the hydrochloride, dihydrochloride, sulfate, bisulfate, and gluconate. All quinine salts can be given by oral or intravenous (IV) routes of administration. Quinine gluconate may also be given intramuscularly (IM) or rectally (PR).

Parenteral quinine (IV and IM) is indicated for the treatment of severe malaria. Parenteral quinine proved safe and effective in the treatment of severe falciparum malaria if rate-controlled intravenous infusion is used. Slow intravenous infusion of quinine dihydrochloride $10 \mathrm{mg} / \mathrm{kg}$ bw over $4 \mathrm{~h}$ is now the standard method of administering quinine in severe malaria. A loading dose of quinine $(20 \mathrm{mg} / \mathrm{kg}$ bw quinine dihydrochloride equivalent to $16.7 \mathrm{mg}$ base), infused over $4 \mathrm{~h}$, has proved to be a safe method of achieving immediate therapeutic plasma concentrations for $P$. falciparum. The loading dose of quinine is however contraindicated in patients with previous treatment with quinine, quinidine, or mefloquine within $48 \mathrm{~h}$. In other areas where the parasite is still sensitive to quinine, a loading dose may not be necessary. In areas with excessive use of quinine, it is too dangerous to give loading dose routinely in every patient. Severe cardiac arrhythmia has been seen in patients who were treated with quinine prior to receiving a loading dose.

In pregnancy, quinine can still be used safely and effectively in the treatment of acute uncomplicated falciparum malaria. No abortion is observed when quinine is administered to pregnant women during the first trimester. In the second and third trimester, increase in uterine contractions and fetal distress may occur during the first $24 \mathrm{~h}$ after the start of treatment when fever is probably still high. A 7-day quinine is strongly recommended for treatment of pregnant women in the first trimester with uncomplicated $P$. vivax, P. ovale, P. malariae, or P. knowlesi. In the first trimester of pregnancy and children aged lower than 8 years, quinine-clindamycin combination is used since tetracyclines are contraindicated. A 7-day oral quinine in combination with tetracycline, doxycycline, or clindamycin is used in the treatment of uncomplicated $P$. falciparum malaria as an alternative treatment when an effective ACT is not promptly available. The addition of these antibiotics improves clinical efficacy of quinine, probably through mechanisms involving both pharmacokinetic and pharmacodynamic interactions, and allows for shortening of treatment regimens. Nevertheless, patients' compliance is the major problem with all these 7-day regimens as it is difficult to achieve without hospitalization. Once the malaria symptoms have subsided, patients are reluctant to continue taking quinine because of cinchonism; poor compliance and subsequent incomplete treatment of patients who remain parasitemic are favorable to the selection of parasites less sensitive to quinine.

\section{Adverse Reactions and Toxicity}

Quinine has a narrow therapeutic window. Serious adverse reactions are infrequent, but minor adverse effects are common. In general, patients with malaria, particularly children, tolerate high blood concentrations of quinine well. Adverse reactions are less frequent than in healthy subjects due to the increased plasma protein binding of quinine in patients with malaria. Most of the adverse reactions are concentration dependent and become more frequent at high plasma concentrations. The characteristic symptom complex of cinchonism commonly occurs, which in its mild form consists of tinnitus, headache, lightheadedness, nausea, vomiting, and slight disturbance of vision. This symptom complex is transient and frequently occurs when plasma quinine levels exceeded $5 \mathrm{mg} / \mathrm{l}$, but it disappears spontaneously after discontinuation of the drug. With the higher doses, these adverse reactions become more severe. The eighth nerve damage occurs with vertigo and decreased auditory acuity. Visual damage with blurred vision, disturbances of color 
perception, night blindness, and diplopia indicate optical nerve involvement. Anorexia, vomiting, constipation, abdominal pain, and diarrhea may occur after therapeutic doses of quinine. These gastrointestinal effects are due both to the local irritant effect of quinine on the gastrointestinal tract and the central effects of quinine on the chemoreceptor trigger zone. Quinine is also a common cause of drug-induced thrombocytopenia and the most common cause of drug-induced thrombotic microangiopathy.

Quinine and its diastereoisomer quinidine including some of the metabolites (3-hydroxyquinidine, 2'-oxyquinidine, and quinidine $\mathrm{N}$-oxide) possess cardiovascular effects. Quinidine is more likely to produce cardiac effects than quinine. The most serious reactions are associated with rapid injection of a large dose, leading to toxic blood concentrations which result in hypotension, cardiac conduction disturbances (heart block, ventricular fibrillation), or even death. When the drugs are administered by slow intravenous infusion or orally, these lifethreatening adverse reactions are rare. The only effects are minor ECG changes (lengthening of the QTc interval, widening of the QRS complex, and T-wave flattening). These potentially dangerous adverse effects must be of concern particularly in patients with history of pre-existing quinine or quinidine therapy. Whenever parenteral therapy is used in severely ill patients, cardiac monitoring is recommended. Young children with severe malaria might be more susceptible to quinine cardiotoxicity than older children.

Cinchona alkaloids stimulate insulin release from pancreatic islet cells, resulting in low levels of blood glucose in malaria patients. However, malarial infection itself may also produce hypoglycemia, particularly in severe infections. During intravenous quinine therapy, potentiation of these effects can be seen more frequent in severe falciparum malaria. Pregnant women and children also appear particularly prone to quinine-induced hypoglycemia. Blood glucose levels should be monitored when the drugs are being used in pregnant patients, children, or severely ill patients.

An overestimated property of quinine is its oxytocic potential to induce premature labor.
Quinine has been used to augment labor and also to induce abortion. However, the abortifacient doses of quinine are two to three times greater than those used for malaria. In addition, it has been demonstrated that malaria itself may have more oxytocic effect than quinine. Pregnancy therefore is not a contraindication to the use of quinine in the treatment of malaria.

Less frequent but more serious adverse reactions of quinine include urticaria, asthma, thrombocytopenia, bronchospasm, angioedema, and hemolysis. Interrupted and recurrent quinine therapy in nonimmune individuals with $P$. falciparum infections seems to predispose them to the complication of blackwater fever, a syndrome of severe hemolytic anemia, hemoglobinuria, oliguria, and jaundice. Occasionally, quinine and its stereoisomer quinidine have also been reported to aggravate weakness in myasthenia gravis. The use of quinine for treatment of cramp up to 60 days can be associated with rare but lifethreatening adverse effects. Its used for this purpose is therefore restricted in some countries.

Quinine poisoning is uncommon but can cause serious retinal and cardiovascular toxicity.

\section{Contraindications}

Quinine is contraindicated in patients with known hypersensitivity to quinine, any of the cinchona alkaloids, or other structurally related drugs.

\section{Caution}

Although there is little evidence of cardiotoxicity in patients with malaria, quinine should be used with caution in patients who have heart rhythm disorders or heart disease. Quinine metabolites may cause oxidative hemolysis, and its use in patients with G6PD deficiency should be with caution. Plasma quinine may accumulate in patients with hepatic or renal diseases, and caution is also advised in treating these patients with quinine.

\section{Pharmacokinetics}

Pharmacokinetic parameters of quinine following currently recommended doses for treatment of uncomplicated and severe malaria are summarized in Table 1. 
Quinine is rapidly absorbed following both oral and parenteral routes. $\mathrm{C}_{\max }$ is reached $1-6 \mathrm{~h}$ after a single oral dose. A loading dose of $20 \mathrm{mg} / \mathrm{kg}$ bw immediately reaches $93 \%$ of $\mathrm{C}_{\max }$ and $75 \%$ of the steady-state trough levels. Rapid intravenous injection results in high toxic concentrations which affect the cardiovascular system. Constant quinine dihydrochloride infusion of $20 \mathrm{mg}$ (salt)/kg bw over $4 \mathrm{~h}$ as a loading dose, followed by $10 \mathrm{mg}$ (salt) $/ \mathrm{kg}$ bw intravascular infusion over $2 \mathrm{~h}$, given 8 hourly for 7 days give satisfactory concentration profiles with reduced risk for cardiovascular toxicity. Intramuscular injection and oral administration produce plasma concentrations lower than those following intravenous infusion. Intramuscular quinine is slowly absorbed with $\mathrm{t}_{\max }$ of about $5 \mathrm{~h}$. A loading dose of $20 \mathrm{mg}$ (salt)/kg bw provides a satisfactory plasma concentration and can be considered as alternative route of administration if the intravenous route is not possible.

Pharmacokinetics of quinine after intravenous injection is generally described by two exponential terms with a rapid distribution phase with a short half-life of about 2 min and a slower $t_{1 / 2}$ similarly to that after the oral dose. The $\mathrm{Vc} / \mathrm{F}$ is approximately one-third of the $\mathrm{Vd} / \mathrm{F}$. Due to this pharmacokinetic property, it is therefore suggested that intravenous administration of quinine should be given by rate-controlled infusion in order to avoid potentially toxic blood concentrations early in the distribution phase.

Quinine is distributed throughout most of the body fluids and is detectable in cerebrospinal fluid (CSF), breast milk, and placenta. Approximately $85 \%$ of the drug is bound to plasma proteins, mainly to $\alpha_{1}$-acid glycoprotein. Unlike chloroquine, and mefloquine, quinine is not concentrated in red cells. The concentration in erythrocytes is between one-fifth and one-third of that in plasma. Quinine is measurable in saliva within a few minutes of oral dosing or intravenous infusion. It remains detectable for up to $36 \mathrm{~h}$ after a $500 \mathrm{mg}$ dose. Saliva concentrations could therefore be used as a noninvasive method to measure patients'compliance. Concentrations in saliva which represent the unbound fraction of quinine are approximately one-quarter to one-third of the plasma concentrations. Quinine concentrationtime profiles for plasma, red cells, and saliva are parallel, giving similar estimation of $t_{1 / 2}$ in all three media. Quinine does not freely cross the blood-brain barrier. The concentrations in CSF have been reported between $2 \%$ and $7 \%$ of the corresponding plasma concentrations. The ratio of $\mathrm{CSF} /$ plasma free quinine concentrations is approximately 0.55 .

Hepatic biotransformation accounts for about $80 \%$ of its total clearance. Quinine undergoes extensive hepatic biotransformation, predominantly via CYP3A4/5 as well as CYP2C9, CYP1A2, and CYP2D6 into several metabolites. 3-hydroxylation mainly via hepatic CYP3A4/5 to its primary metabolite, 3-hydroxyquinine (Fig. 4b), has been shown to contribute $5-10 \%$ of the antimalarial activity. Formation of the minor metabolites (10S)-11dihydroxydihydroquinine and 2L'-quininone) is also dependent on CYP3A4/5, while the formation of (10R)-11-dihydroxydihydroquinine might be linked to CYP2C9. The drug and its metabolites appear in the urine within $1 \mathrm{~h}$ of drug administration, and little remains in the body after $48 \mathrm{~h}$. Elimination from the body is rapid with $\mathrm{CL} / \mathrm{F}$ of about $0.2-5 \mathrm{ml} / \mathrm{min} / \mathrm{kg}$. About $20 \%$ of quinine is excreted unchanged in the urine, and small amounts may appear in the bile and saliva. Renal excretion involves by both glomerular filtration and tubular secretion. There appears to be stereoselective net renal tubular secretion of quinidine over quinine which indicates stereoselectivity of the renal tubular transport process.

\section{Factors Associated with Altered Drug Exposure and/or Treatment Response}

Malaria Infection The pharmacokinetics of quinine is significantly altered by malaria infection. The absorption is not affected, but the $\mathrm{CL} / \mathrm{F}$ is reduced from 0.2 to $5 \mathrm{ml} / \mathrm{min} / \mathrm{kg}$ in healthy subjects to about 1.4 and $0.9 \mathrm{ml} / \mathrm{min} / \mathrm{kg}$ in uncomplicated malaria and cerebral malaria, respectively. The excretion of quinine is inversely related to disease severity and most impeded in severe malaria infection. It is likely that the main factor that influences the decreased clearance of quinine is the impairment of CYP3A4/5 function. The Vd 
is contracted $(1.1 \mathrm{l} / \mathrm{kg})$ in cerebral malaria. The $t_{1 / 2}$ is prolonged during acute infection with a mean of $16 \mathrm{~h}$ or even longer in severe malaria $(18 \mathrm{~h})$. Consequently, elevation of plasma drug concentrations is observed which is also proportional to the severity of the disease. Despite such high plasma concentrations in severe falciparum malaria, there is no apparent quinine toxicity. This is explained by the increase in plasma protein binding of quinine to $\alpha_{1}$-acid glycoprotein in patients with cerebral malaria (93\%). The extent of increase in plasma protein binding of quinine is relatively lower in uncomplicated malaria (90\%). Despite the marked reduction of quinine clearance in severe malaria, maintenance dose reduction is not recommended in the initial phase of treatment because severe infections cause high early fatality rates. The danger of inadequate treatment overweighs the risk of toxicity. The dose should be reduced in severe malaria only when there is evidence of severe cardiotoxicity or persistent renal failure after 3 days of treatment (by this time enough quinine is available for consistent parasiticidal activity). Dose reduction by one-third after 3 days has been suggested to prevent further rise in plasma quinine concentrations in such cases. In the presence of severe arrhythmia, discontinuation of quinine treatment should be considered if effective alternative drugs such as artemisinin drugs are available.

Children The disposition of quinine changes with age with slightly higher concentrations observed in children aged lower than 2 years. In children with malaria, the $\mathrm{Vd}$ is contracted $(30 \%$ smaller) and the CL is increased. As a result of these changes, shorter $t_{1 / 2}$ is observed in children. The decline in plasma concentrations of quinine in the latter half of the treatment course $(10 \mathrm{mg}$ base/ $\mathrm{kg}$ bw every $8 \mathrm{~h}$ for 7 days) is associated with treatment failure when values fall below the putative minimum inhibitory concentration (MIC). For this reason it has been suggested that the individual dose of quinine in children should be increased to $15 \mathrm{mg}$ base $/ \mathrm{kg}$ body weight in the second half of the treatment course.
Elderly Subjects Disposition kinetics of quinine is altered in healthy elderly subjects when compared to those of younger adult subjects. The rate of drug absorption is not altered, but the $\mathrm{CL} / \mathrm{F}$ is significantly reduced (26\% reduction). The lower $\mathrm{CL} / \mathrm{F}$ in the elderly group indicates the decreased hepatic biotransformation of quinine in old age, since the renal clearance of drug is not altered. This results in a prolongation of the $t_{1 / 2}$ (about $18.4 \mathrm{~h}$ ). Plasma protein binding of quinine remains unchanged despite the lower plasma albumin. This is due to the fact that quinine binds extensively to $\alpha_{1}$-acid glycoprotein rather than albumin. Significant drug accumulation may occur after multiple dosing of malaria treatment. The clinical significance of this finding is unclear, but it emphasizes the need for caution in the administration of quinine to elderly patients.

Malnutrition Intestinal malabsorption is a feature of kwashiorkor. This condition significantly affects the pharmacokinetics of quinine. The longer apparent absorption half-life $\left(t_{1 / 2 a}\right)$ and $t_{\max }$ and the lower $\mathrm{C}_{\max }$ observed in patients with kwashiorkor suggest slower and lower absorption of quinine in this group of patients, although the contribution from differences in distribution kinetics cannot be excluded. Quinine is eliminated more slowly in children with kwashiorkor, and therefore, $t_{1 / 2}$ is significantly prolonged. Enlarged fatty liver is common in kwashiorkor, and this is associated with reduced activity of some of the oxidative liver enzymes.

Pregnancy Quinine is one of the first-line drugs recommended for use during the first trimester of pregnancy. The pharmacokinetic properties of quinine are not different between pregnant and nonpregnant women with uncomplicated malaria. In severe malaria, the $\mathrm{Vd} / \mathrm{F}$ is generally reduced by about $30 \%$, and elimination is more rapid. Alterations in plasma protein and tissue protein binding associated with malaria infection and pregnancy itself may be responsible for the reduction in the Vd. Placental cord plasma quinine concentrations range between 1 and $4.6 \mathrm{mg} / \mathrm{l}$, which correlate well with maternal plasma quinine concentrations. The mean ratio of cord 
plasma to maternal plasma quinine concentration is 0.32 and considered safe for breast-feeding. Breast milk-to-plasma ratio ranges from 0.11 to 0.53 .

Hepatic Diseases Hepatic metabolism of quinine is reduced in hepatic insufficiency. Pharmacokinetics of quinine during the acute phase of hepatitis-B infection is significantly different from those in healthy subjects. The $t_{1 / 2}$ is prolonged $(17 \mathrm{~h})$ and $\mathrm{CL} / \mathrm{F}$ is reduced. However, the pharmacokinetics obtained during acute hepatitis is not different from those during the recovery phase. Despite a return of liver function tests to normal during convalescence after hepatitis, the clearance of quinine remains impaired. This suggests that curative regimens of quinine used in the routine treatment of falciparum malaria may not be suitable for malaria patients with acute hepatitis or even those who have had hepatitis within the past 3 months. The combined effects of acute hepatitis and malaria on the kinetics of quinine suggest caution with its dosage in patients with both conditions.

Renal Diseases Quinine clearance is reduced in acute renal failure complicating malaria, but this is the result of pharmacokinetic changes related to the acute infection rather than due to reduced renal function per se, as urinary quinine clearance comprises only $20 \%$ of the total clearance. Hemofiltration has no significant influence on the total body clearance of quinine.

\section{Genetic Polymorphisms of Drug-Metabolizing}

Enzymes Substantial variation of quinine pharmacokinetics between individuals was observed in children from Ghana with severe malaria. The possible involvement of polymorphisms of drugmetabolizing enzymes has been proposed but remains to be confirmed. One study showed that 3-hydroxylation metabolite of quinine is substantially lower in healthy people from Tanzania harboring the $C Y P 3 A 5^{*} 3 / * 3$ low-expression genotype.
Drug Interactions Various studies suggest significant pharmacodynamic and pharmacokinetic interactions between quinine and other drugs including other antimalarial drugs. The QTc interval may be prolonged when quinine is given with antiarrhythmic drugs such as flecainide and amiodarone. In addition, ventricular arrhythmia may occur when the drug is given with antihistamines (e.g., terfenadine) or antipsychotic drugs (e.g., thioridazine). Quinine has been shown to potentiate the oral anticoagulants by inhibition of prothrombin synthesis.

With regard to pharmacokinetic interactions, systemic exposure of quinine is unchanged when quinine is co-administered with sulfadoxinepyrimethamine (SP) and oral contraceptive steroids. On the other hand, plasma quinine concentrations are increased when co-administered with tetracycline, quinidine/cinchonine, cimetidine, ketoconazole, omeprazole, nifedipine, troleandomycin, and erythromycin. The mechanism involved is mainly through hepatic CYP450 inhibition. Plasma quinine concentrations in the presence of tetracycline are significantly higher than those with quinine alone. The addition of the course of tetracycline $(250 \mathrm{mg}$, 6 hourly for 7 days) to the conventional regimen of quinine (600 mg 8 hourly for 7 days) has an influence on the maintenance of plasma quinine concentrations above the MIC throughout the 7-day period of treatment. Higher plasma concentrations of each component have also been observed when quinine, quinidine, and cinchonine were given in combination.

Drugs which have been shown to reduce systemic exposure of quinine include rifampicin, isoniazid, lopinavir/ritonavir (LPVr), and activated charcoal. Acidification of the urine also increases the excretion rate of quinine by about twofold resulting in low systemic exposure. Rifampicin and isoniazid decrease plasma quinine concentrations, possibly by the induction of hepatic metabolism of quinine. Following 1 week pre-treatment with rifampicin and isoniazid, the $\mathrm{CL} / \mathrm{F}$ of quinine is significantly increased, and the $t_{1 / 2}$ is shortened. The combined therapy of quinine and rifampicin for P. falciparum, however, resulted in faster parasite clearance which could be due to antimalarial 
activity of rifampicin rather than drug interaction, since induction of CYP3A4-mediated quinine metabolism activity by rifampicin takes several days. Quinine penetrates relatively poorly into the cerebrospinal fluid (CSF) in patients with cerebral malaria, with a concentration of approximately $2-7 \%$ of plasma concentrations. Co-administration of rifampicin and quinine may also decrease quinine concentrations in the CSF due to inducing effect of rifampicin on the efflux protein P-glycoprotein. Rifampicin should, therefore, not be combined to antimalarial for malaria treatment despite its intrinsic antimalarial activity.

Concomitant use of antimalarial and antiretroviral drugs is increasingly frequent in malaria and HIV-endemic regions. The reduction in systemic exposure of quinine and 3-hydroxyquinine with concomitant LPV/r use (ritonavir-boosted lopinavir) raises concerns of suboptimal exposure. The rate of elimination of a therapeutic dose of quinine is increased when activated charcoal is administered at regular intervals commencing $4 \mathrm{~h}$ after therapeutic dose of quinine $(600 \mathrm{mg})$. Activated charcoal shortens quinine $t_{1 / 2}$ and increases CL by approximately $50 \%$. Recently, it has been shown that repeated oral charcoal (50 g, 4 hourly) is highly effective and is only one approach in enhancing the removal of quinine in symptomatic patients with acute quinine poisoning to reduce the risk of potentially dangerous complications.

Quinine inhibits the activity of CYP2D6 as well as P-glycoprotein and biliary excretion. It has been shown to interact with antipyrine, digoxin, ampicillin-cloxacillin, and rifampicin upon co-administration. Quinine inhibits biliary excretion and may theoretically alter rifampicin $\mathrm{t}_{1 /}$ 2. Chronic administration of quinine shortens plasma antipyrine $t_{1 / 2}$. Quinine increases digoxin plasma levels, probably by reducing its nonrenal clearance. Quinine reduced the bioavailability and the antimicrobial activity of ampicillin-cloxacillin upon co-administration, which may have therapeutic implications. The MIC of both antibiotics in the presence of quinine was five- to sevenfold increased, indicating a decrease in antimicrobial activity by quinine.

\section{Mefloquine}

During the Second World War, the US Army initiated a program for the discovery and development of new antimalarial drugs. The most promising chemical class to emerge from this extensive research program was found to exhibit phototoxic adverse effects, and thus its further development was precluded. In 1960, following the emergence of chloroquine-resistant P. falciparum in Southeast Asia, the search continued, and the compound selected from this class was WR 142490. It was proved both to be safe and reliably effective. This compound was later named mefloquine. Since then, the development of mefloquine was continued by the World Health Organization (WHO) in collaboration with Hoffmann-La Roche and the US Walter Reed Institute of Research (WRIR). The initial phase I and II clinical trials were carried out between 1972 and 1978 in many tropical countries including Zambia, Brazil, Vietnam, and Thailand. Results showed mefloquine to be safe, generally well tolerated, and therapeutically effective in a single dose of 750-1500 mg against both chloroquine-resistant and chloroquine-sensitive P. falciparum, with initial cure rates approaching $100 \%$.

\section{Chemistry and Physical Properties}

Mefloquine (Fig. 5a) is a 4-quinolinemethanol derivative which has two asymmetric carbon atoms in the molecule and is used clinically as a racemic mixture (50:50) of the erythro isomers (dextrorotatory 11R, 2'S and levorotatory 11S, 2'R).

Mefloquine is poorly soluble in water $(10 \mathrm{~g} / 1$ at $6^{\circ} \mathrm{C}$ ). The molecular weight of the base is 378 . The hydrochloride salt (MW 415) is a white, odorless, and bitter tasting powder. It is slightly soluble in water. The drug has a tendency to bind to cell membranes, proteins, and plastics.

\section{Pharmacological Activities}

Antimalarial Activity and Mechanism of Action and Resistance Mefloquine is a potent and long-acting blood schizontocidal antimalarial against all human species of Plasmodium including multidrug-resistant strains of $P$. falciparum. In 
Pharmacology of

Antimalarial Drugs,

Current Anti-malarials,

Fig. 5 Chemical structures

of (a) mefloquine and

(b) carboxymefloquine

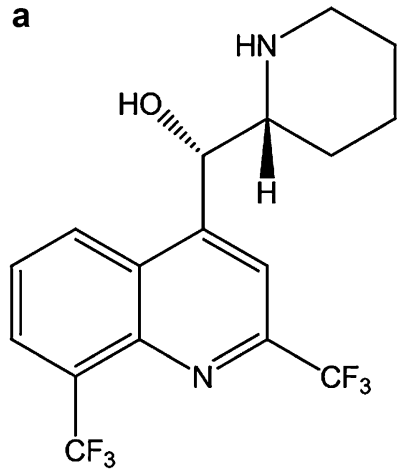

b

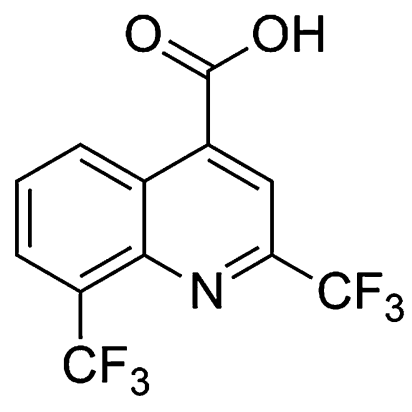

addition, it is active against gametocytes of $P$. vivax, $P$. ovale, $P$. malariae, and $P$. knowlesi. However, it has no effect against exoerythrocytic forms of malaria. Its major plasma metabolite carboxymefloquine (Fig. 5b) has no significant antimalarial activity. Mefloquine exhibits approximately the same stage specificity of action as quinine, killing primarily the large rings and trophozoites of asexual parasites.

The exact modes of antimalarial action and resistance of mefloquine remains unclear. It is thought that mefloquine may share some mechanisms with chloroquine and quinine. Three possible mechanisms of action of mefloquine have been proposed: the impairment of NADPH oxidation process through its interaction with phospholipids in the parasite membrane, the interaction with ferriprotoporphyrin IX (FPIX), and the action on the parasite food vacuoles, raising intravesicular $\mathrm{pH}$ and thereby interfering with food digestion of the parasites. A more recent proposal is inhibition of endocytosis of the cytosol by the parasite.

Mefloquine was first introduced as first-line treatment for $P$. falciparum malaria in Thailand in 1984. However, despite its restricted clinical uses, significant resistance developed within 6 years. Resistance is now spread to several malaria-endemic areas particularly Southeast Asian countries. Resistance of P. falciparum to mefloquine is shown to be mediated by amplification of $p f m d r l$, leading to overexpression of the encoded digestive membrane transporter PfMDR1. Studies on transgenic parasites later on demonstrate that reduced parasite susceptibility to mefloquine is associated with increased pfMDR1-mediated import into parasite food vacuole. This suggests that a primary target (s) of action of mefloquine resides outside digestive food vacuole. Inhibition of the import of many compounds into the food vacuole by mefloquine has been shown.

Other Pharmacological Activities and Clinical Uses Mefloquine exerts in vitro and in vivo activity against Mycobacterium avium complex including strains with multidrug resistance. In addition, it is also found to be effective against Schistosomes. A single dose of mefloquine possesses potential effect against three major species of schistosomes (Schistosoma mansoni, Schistosoma haematobium, and Schistosoma japonicum) infecting humans. When used as IPTp for malaria prevention, mefloquine shows promising activity against concomitant $S$. haematobium leading to reduction of egg excretion in pregnant women.

\section{Therapeutic Indications for Malaria}

Mefloquine is currently recommended by WHO for the chemoprophylaxis of malaria caused by all Plasmodium species. In addition, it is also recommended for treatment of uncomplicated malaria in combination with artesunate (one of the ACTs). As there is no parenteral form of mefloquine, the drug is generally not used in severe malaria. In addition, mefloquine is contraindicated in cerebral malaria due to $\mathrm{CNS}$ toxicity. 
For prophylaxis, mefloquine is likely to be an effective chemoprophylactic agent for long-term use (4-6 weeks). Its use is recommended for 6 months and beyond with clinical controls. It is the only prophylactic drug recommended for firsttrimester pregnancies and considered safe throughout pregnancy. The dose of $250 \mathrm{mg}$ base (274 mg mefloquine hydrochloride) weekly has been recommended, but the maintenance of this dose level beyond 12 weeks may entail accumulation and increase incidence of adverse reactions.

For treatment of uncomplicated malaria, a total therapeutic dose of $4(2-10) \mathrm{mg} / \mathrm{kg}$ bw per day artesunate and $8.3(5-11) \mathrm{mg} / \mathrm{kg}$ bw per day mefloquine, given once a day for 3 days, is recommended. Formulations are currently available as fixed-dose artesunate-mefloquine combination as pediatric tablets containing $25 \mathrm{mg}$ artesunate and $55 \mathrm{mg}$ mefloquine hydrochloride (50 mg base) and adult tablets containing $100 \mathrm{mg}$ artesunate and $220 \mathrm{mg}$ mefloquine hydrochloride (200 mg base). To reduce vomiting, mefloquine dose should be split over 3 days as in current fixed-dose combination.

\section{Adverse Reactions and Toxicity}

Mefloquine is generally well tolerated when given alone or in combination with artesunate. The majority of adverse reactions after therapeutic or prophylactic dose of mefloquine are mild, transient, and require no specific treatment. Severe adverse reactions are rare. Adverse reactions appear to be associated with high concentrations of the (-) enantiomer rather than the racemic mefloquine and are more frequent in females than males.

Nausea, vomiting, abdominal pain, and diarrhea are the most common adverse effects after mefloquine in patients with malaria. Nausea and vomiting are dose related, being more likely in adult patients receiving higher dosage than $15 \mathrm{mg} / \mathrm{kg}$ bw. Vomiting is rarely observed in healthy subjects after drug administration. Onset of nausea and vomiting from mefloquine is rapid (within the first few hours after drug administration); it is therefore suspected to be the result of a local effect (i.e., gastric irritation) rather than a central effect of mefloquine. In addition, nausea is unlikely to be a function of mefloquine blood concentrations as there is evidence that the incidence of this symptom is decreased when an antiemetic, metoclopramide, is given prior to mefloquine administration, despite an increase in $\mathrm{C}_{\max }$ and systemic exposure of mefloquine. Vomiting within the first hour after drug administration has been shown to result in low plasma concentrations which lead to treatment failure. Diarrhea after mefloquine is usually mild in the majority of cases, occurring in $10-50 \%$ of patients who are treated with mefloquine, with an average duration of 2-3 days.

Mefloquine has been associated with seizures, anxiety, irritability, dizziness, paranoia, suicidal ideation, depression, hallucinations, and violence during therapeutic dose regimen and long-term malaria prophylaxis. Such neuropsychiatric reactions generally resolve after discontinuation of the drug. The estimated incidence of seizures, encephalopathy, or psychotic reactions ranges from 1 in 10,000 healthy people receiving chemoprophylaxis, 1 in 1000 malaria patients in Asia, 1 in 200 malaria patients in Africa, to 1 in 20 patients recovering from cerebral malaria.

Less frequently reported adverse reactions include effects on blood and lymphatic (agranulocytosis, aplastic anemia), nervous (syncope, convulsions, abnormal coordination, memory impairment, sensory and motor neuropathies including paresthesia, tremor and ataxia, and encephalopathy), cardiovascular (tachycardia, palpitation, QTc prolongation, bradycardia, irregular heart rate, extrasystoles, A-V block, and other transient cardiac conduction alterations), eye and ear (visual disturbances, tinnitus, and hearing impairment), gastrointestinal (dyspepsia), and immune (hypersensitivity reactions ranging from mild cutaneous events to anaphylaxis) systems. Hypersensitivity reactions including anaphylaxis and pneumonitis have also been associated with the use of mefloquine. As with most medications, hypersensitivity reactions, ranging from mild cutaneous events to anaphylaxis, cannot be predicted.

Rare reactions include edema, chest pain, asthenia, malaise, fatigue, chills, pyrexia, anorexia, muscle weakness, muscle cramps, myalgia, arthralgia, drug-related hepatic disorders 
(from asymptomatic transient transaminase elevations to hepatic failure), and blood disorders (decreased hematocrit, transient elevation of transaminases, leukopenia or leukocytosis, and thrombocytopenia). There has been no evidence of hemolytic effect of mefloquine in subjects with G6PD deficiency.

Mefloquine is not mutagenic or carcinogenic. However, it is teratogenic and embryotoxic in experimental animal. However, data from published studies in pregnant women have shown no increase in the risk of teratogenic effects or adverse pregnancy outcomes following mefloquine treatment or prophylaxis during pregnancy. Prophylactic doses of mefloquine in the second or third trimesters of pregnancy appear to be effective and are not associated with adverse maternal or fetal outcomes. However, gastrointestinal adverse reactions including nausea and vomiting are common in pregnant women treated with mefloquine.

\section{Contraindications}

Mefloquine is contraindicated in patients with a known hypersensitivity to mefloquine or structurally related compounds, (e.g., quinine, quinidine, chloroquine, or amodiaquine). The drug should not be prescribed for follow-up treatment after cerebral malaria. Mefloquine prophylaxis is contraindicated in patients with active depression or a history of psychiatric disturbances (including depression, generalized anxiety disorder, psychosis, schizophrenia, or other major psychiatric disorders) or a history of convulsions, since the drug may precipitate these conditions.

\section{Caution}

Patients with hepatic impairment who receive mefloquine should be monitored carefully for the potential of the increased risk of adverse reactions. Caution should also be made in administering mefloquine to patients with cardiac disease or in concurrent with drugs that alter cardiac conduction (e.g., antiarrhythmics, $\beta$-adrenergic blocking agents, calcium channel blockers, antihistamines (astemizole, terfenadine), tricyclic antidepressants, phenothiazines, and the antimalarial drugs quinine, halofantrine, and mefloquine). As small amount of mefloquine is excreted in breast milk, the use of mefloquine in nursing women should be with caution.

\section{Pharmacokinetics}

Pharmacokinetic parameters of mefloquine following currently recommended doses for prophylaxis and treatment of uncomplicated malaria are summarized in Table 1.

Oral absorption of mefloquine is relatively slow. The absolute oral bioavailability of mefloquine cannot be determined since an intravenous formulation is not available. The bioavailability of the tablet formulation compared with an oral solution is over $85 \%$. When taking mefloquine with food, the extent and rate of absorption of mefloquine are increased leading to about a $40 \%$ increase in bioavailability, increased in $\mathrm{C}_{\max }$, and shortened $t_{\max }$. Plasma concentrations peak 8-15 h after a single oral dose of mefloquine. There is considerable enterohepatic circulation impacting on $\mathrm{t}_{\max }$ and $\mathrm{C}_{\max }$ leading to high variability of plasma/blood concentrations. At the prophylactic dose of $250 \mathrm{mg}$ once weekly, maximum steady-state plasma concentrations of $1000-2000 \mathrm{ng} / \mathrm{ml}$ are reached after 7-10 weeks.

Mefloquine is extensively bound to plasma proteins and also to tissue and red cell membranes. Plasma protein binding is $98 \%$, mainly to $\alpha_{1}$-acid glycoprotein. The $\mathrm{Vd} / \mathrm{F}$ is relatively large, indicating extensive tissue distribution. Mefloquine may accumulate in parasitized erythrocytes at an erythrocyte-to-plasma concentration ratio of about 2. Mefloquine also crosses the placenta.

The biotransformation of mefloquine occurs in the liver by CYP3A4 into two inactive metabolites, carboxymefloquine (2-8-bis-trifluoromethyl-4quinoline carboxylic acid: Fig. 5b) and hydroxymefloquine. The main metabolite carboxymefloquine is inactive against $P$. falciparum. This metabolite appears in plasma $2-4 \mathrm{~h}$ after a single oral dose of mefloquine. Its concentrations rise steadily and exceed those of the patent drug after in about 2-3 days. $\mathrm{C}_{\max }$ of carboxymefloquine in plasma, approximately three to five times higher than that of mefloquine, is reached after 2 weeks. Carboxymefloquine is eliminated from plasma at a rate that is similar to that of mefloquine. The 
relatively high plasma concentration of carboxymefloquine is explained by its small $\mathrm{Vd} / \mathrm{F}$. It is unlikely that much of carboxymefloquine penetrates the CNS, as it is not detectable in the CSF.

Mefloquine has been shown to be a substrate and/or inhibitor of the transporters P-glycoprotein (MDR1), multidrug resistance protein (MRP), as well as breast cancer receptor protein (BCRP). This is important because MRP1 and MRP4 (and MRP5 and BCRP) in red blood cells might affect red cell mefloquine concentrations and drug effectiveness as well as the development of drug resistance to mefloquine. By contrast, MDR1 might be more important in establishing mefloquine safety as it controls mefloquine concentrations and entry of the drug into the brain.

The $t_{1 / 2}$ of mefloquine varies between 2 and 4 weeks with an average of about 3 weeks. The value remains unchanged during long-term prophylaxis. Mefloquine is excreted into bile and feces. Urinary excretion of unchanged mefloquine and carboxymefloquine accounts for about $9 \%$ and $4 \%$ of the dose, respectively. Concentrations of other metabolites could not be measured in the urine. Low concentrations (3-4\%) of mefloquine are excreted in breast milk following a dose equivalent to $250 \mathrm{mg}$ of the free base.

The multiple-dose kinetics of mefloquine is similar to single-dose pharmacokinetics. This suggests that there is no auto-induction or autoinhibition of the metabolic clearance mechanisms of mefloquine. The pharmacokinetics of mefloquine is also highly stereospecific. Following the administration of a single oral dose of $1000 \mathrm{mg}$ mefloquine to a healthy male Caucasian, plasma and whole blood concentrations of $\mathrm{R}(-)$ mefloquine were greater than those of the $\mathrm{S}(+)$ enantiomer. The ratios of $\mathrm{R}(-)$ and $\mathrm{S}(+)$ mefloquine in plasma and whole blood ranged from 1.7 at $2 \mathrm{~h}$ to 11.5 at $504 \mathrm{~h}$ and from 1.5 at $2 \mathrm{~h}$ to 3 at $504 \mathrm{~h}$, respectively.

\section{Factors Associated with Altered Drug Exposure and/or Treatment Response}

Malaria Infection Pharmacokinetics of mefloquine appears to be altered in malaria. Absorption of the drug is usually not changed in uncomplicated falciparum malaria. In severely ill patients, i.e., cerebral malaria, absorption may be incomplete despite the apparent rapidity in the rate of absorption. The $\mathrm{Vd} / \mathrm{F}$ is contracted and $\mathrm{CL} / \mathrm{F}$ is reduced during an acute phase of malaria infection. However the effect on clearance is less predictable as the elimination rate is increased in patients with malaria. Indirect evidence from a few studies suggests that the shorter $t_{1 / 2}$ of mefloquine in patients with uncomplicated falciparum malaria may be caused by an interruption of enterohepatic recycling (EHC) of mefloquine in malaria. The pharmacokinetic alterations resulting from the interruption of EHC by broadspectrum antibiotics ampicillin and tetracycline closely resemble those observed in patients with uncomplicated malaria.

Ethnics Pharmacokinetic variability of mefloquine has been observed among various ethnic populations. Mefloquine concentration in healthy Thai subjects is higher than that in Caucasian subjects, while comparable pharmacokinetics is reported among Brazilian, Caucasian, and African subjects. In practice, however, these are of minor importance with regard to drug efficacy.

Children No relevant age-related changes have been observed in the pharmacokinetics of mefloquine. Clinical experience has not identified differences in responses between the elderly and younger patients. Children with uncomplicated falciparum malaria showed a good absorption with blood concentrations comparable to those seen in adults.

Pregnancy Although the rate of mefloquine absorption in late pregnancy is unchanged, $\mathrm{C}_{\max }$ and systemic availability are significantly reduced during the first 2 days of mefloquine administration. The oral bioavailability of mefloquine in late pregnancy may be altered as a result of delayed gastric emptying, decreased motility of the gastrointestinal tract, or changes in plasma/tissue protein binding. The expansion of the $\mathrm{Vd} / \mathrm{F}$ or the increase in CL/Fcould be due to an increase in the glomerular filtration rate. Suboptimal dosing 
could partially explain the poorer treatment responses observed among pregnant women.

\section{Genetic Polymorphisms in Drug Trans-} porters A prospective clinical trial in Caucasian travelers taking mefloquine prophylaxis.

revealed the link between polymorphisms of the P-gp (MDR1) efflux protein in the brain and incidence of neuropsychiatric adverse reactions of mefloquine. Individuals with genetic defect in the $m d r 1$ gene (ABCB11236TT/2677TT/3435TT haplotype) were associated with a particularly high risk of neuropsychiatric reactions, which were not related to mefloquine serum concentrations. A lower expression of MDR1 in individuals carrying $A B C B 1 T$ variants resulted in lower mefloquine efflux from the brain, exposing individuals to high tissue concentrations related to neuropsychiatric symptoms. This finding might suggest the important role of local MDR1 expression at the blood-brain barrier which leads to the accumulation of mefloquine in the brain without affecting systemic exposure. This genetic polymorphism might also affect stereoselectivity of MDR1 and consequently cerebral or plasma mefloquine (R)/(S) ratio without changing total plasma concentrations. As mefloquine is also a potent MDR1 inhibitor, propensity of drug interaction may occur when mefloquine is used concurrently with other drugs that are substrates or inhibitors of MDR1 (e.g., HIV protease inhibitors, MDR1 substrates).

Renal Diseases No pharmacokinetic study has been performed in patients with renal insufficiency since only small proportion of the drug is eliminated renally. Mefloquine and carboxymefloquine are not significantly removed by hemodialysis.

Hepatic Diseases The pharmacokinetics of mefloquine in patients with compromised hepatic function has not been investigated. Mefloquine is extensively metabolized in the liver by the CYP450 system, and it is possible that the elimination of mefloquine may be prolonged in patients with impaired hepatic function, leading to higher plasma levels.
Drug Interactions The use of drug combinations has shown to delay in emergence of mefloquine resistance in vivo in animals and in vitro when used with sulfadoxine-pyrimethamine (MSP) and with primaquine. Combination of artemisinin with mefloquine has been shown to produce potentiation in vitro, in vivo in mice infected with resistant strains, and in humans presenting with complicated falciparum malaria. The combination of mefloquine with chloroquine or desethylchloroquine exhibited pronounced antagonistic effect in vitro with both chloroquine-sensitive and chloroquine-resistant P. falciparum. Clinically significant QTc interval prolongation has not been reported with mefloquine alone. However, concomitant administration of mefloquine, quinine, quinidine, chloroquine, or halofantrine may produce electrocardiographic abnormalities. Inhibition of KvLQT1/minK in the human heart by mefloquine may in part explain the synergistic prolongation of QTc interval observed. Caution should also be made with other drugs that alter cardiac conduction (see also the section "Caution") since they may contribute to a prolongation of the QTc interval. Concomitant administration of mefloquine with drugs known to lower the epileptogenic threshold (antidepressants, bupropion, antipsychotics, tramadol, quinine, quinidine, or chloroquine) may increase the risk of convulsions.

When mefloquine is taken concurrently with oral live typhoid vaccines, attenuation of immunization cannot be excluded. Vaccinations with attenuated live bacteria should therefore be completed at least 3 days before the first dose of mefloquine.

Blood (plasma or whole blood) concentrations of mefloquine have been shown to be increased when co-administered with metoclopramide, ketoconazole, quinine, ampicillin, and tetracycline. The risk of QTc prolongation and other adverse reactions of mefloquine may also be expected if these drugs are taken during mefloquine therapy for prophylaxis or treatment of malaria. The antiemetic metoclopramide, through stimulation of gastric emptying rate, increases the absorption of mefloquine, resulting in higher $\mathrm{C}_{\max }$ and $\mathrm{AUC}$ in the first $24 \mathrm{~h}$ of treatment. Metoclopramide would seem to have an important role in prevention of vomiting before antimalarial administration in 
malaria patients. Co-administration of ketoconazole, a strong inhibitor of CYP3A4, increases plasma concentrations of mefloquine (AUC by $79 \%$ and $\mathrm{C}_{\max }$ by $64 \%$ ) and prolongs $\mathrm{t}_{1 / 2}$ (by $39 \%$ ). Mefloquine concentration has been reported to rise abruptly after the cessation of quinine administration. Competition for plasma and red cell binding site(s) of mefloquine might explain this interaction. Cardiotoxicity is therefore a major concern as there is a report of the sudden death of a patient who concurrently administered mefloquine and quinine.

In the chemotherapy of malaria, tetracycline has an important role in the treatment of falciparum malaria when given in combination with other blood schizonticides. When tetracycline is given in combination with quinine, chloroquine or sulfadoxine-pyrimethamine, and mefloquine, the cure rate is improved. Pharmacokinetic interaction studies demonstrated that the $\mathrm{t}_{1 / 2}$, mean residence time (MRT) and apparent volume of distribution at steady state $\left(\mathrm{Vd}_{\mathrm{ss}} / \mathrm{F}\right)$ of mefloquine are all reduced when co-administered with tetracycline. In addition, $\mathrm{C}_{\max }$ and AUC are increased during the course of tetracycline administration. Competition between these drugs for biliary excretion could conceivably occur.

Systemic exposure of mefloquine has been shown to be significantly decreased in the presence of rifampicin. Rifampicin induces mefloquine metabolism, decreasing its $\mathrm{AUC}$ by $68 \%$ and $t_{1 / 2}$ by $63 \%$. The AUC and $C L / F$ of carboxymefloquine metabolite are increased by $30 \%$ and $25 \%$, respectively. Simultaneous administration of mefloquine and rifampicin should therefore be avoided.

The concomitant administration of the antimalarials primaquine, sulfadoxine-pyrimethamine as well as oral contraceptive steroids, and antipyrine does not alter the pharmacokinetics or adverse reaction profile of mefloquine. Pharmacokinetics of oral dihydroartemisinin and mefloquine when given concurrently are similar, except for the absorption rate of mefloquine which is faster in the presence of dihydroartemisinin. The pharmacokinetics of artemether, dihydroartemisinin, lumefantrine, and mefloquine are also unchanged when mefloquine is co-administered with artemether or artemetherlumefantrine combination.
Mefloquine has been reported to interact with other drugs when given concurrently. Mefloquine reduces plasma concentration of the anti-HIV ritonavir. Concomitant administration of mefloquine and anticonvulsants may reduce seizure control by lowering the plasma levels of the anticonvulsants. Therefore, patients concurrently taking anticonvulsant medication, including valproic acid, carbamazepine, phenobarbital, phenytoin, and mefloquine, should have the blood level of their anticonvulsants monitored and the dosage adjusted appropriately.

A single case in the literature reports a transient severe psychiatric disturbance, suggesting an adverse reaction to mefloquine associated with a heavy ingestion of alcohol ( $600 \mathrm{ml}$ of whisky).

Carboxymefloquine has been shown to induce drug-metabolizing enzyme and transporter expression by activation of pregnane $\mathrm{X}$ receptor in vitro. Thus, the clinical use of mefloquine may result in pharmacokinetic drug-drug interactions via its metabolite carboxymefloquine. Whether these in vitro findings are of clinical relevance has to be addressed in future clinical drug-drug interaction studies.

\section{Antifolates}

Antifolate drugs include various combinations of dihydrofolate reductase (DHFR) enzyme inhibitors, such as pyrimethamine, proguanil, chlorproguanil, and cycloguanil, and dihydropteroate synthase (DHPS) enzyme inhibitors, such as sulfadoxine, sulfalene, and dapsone. Currently, only the sulfadoxine-pyrimethamine antifolate combination and the combination of antifolate proguanil and atovaquone are recommended for malaria treatment and/or prophylaxis.

\section{Sulfadoxine-Pyrimethamine}

\section{Chemistry and Physical Properties}

Sulfadoxine (4-Amino-N- $\left\{5,6-b i s\left[\left(\sim 2 \sim H \_3\right]\right)\right.$ methyloxy]pyrimidin-4-yl $\}$ benzene-1-sulfonamide: Fig. 6a) appears as white or creamy white, almost odorless crystalline powder. The molecular weight is 310.3. It is not dissolved well in water. 
Pyrimethamine (2,4-Diamino-5-(4-chlorophenyl)-6-ethylpyrimidine: Fig. 6b) also appears as white, almost odorless crystalline powder. The molecular weight is 248.7 .

\section{Pharmacology}

Antimalarial Activities and Mechanisms of Action and Resistance Sulfadoxine is a sulfonamide antibacterial which acts by inhibiting the activity of dihydropteroate synthase (DHPS) and, therefore, synthesis of folic acid by bacteria and malaria parasite. On the other hand, pyrimethamine inhibits dihydrofolate reductase (DHF) and thereby the synthesis of folic acid by bacteria and malaria parasite. Both are active mainly against the later development stages of asexual Plasmodium parasite.

Other Pharmacological Properties The combination of sulfadoxine and pyrimethamine is used in the treatment of toxoplasmosis.

\section{Therapeutic Indications for Malaria}

Sulfadoxine-pyrimethamine is indicated in areas of moderate-to-high malaria transmission intensity for intermittent preventive treatment (IPT) of malaria in pregnant women and in infants. It is also used in combination with amodiaquine for seasonal malaria chemoprevention in children in areas with highly seasonal malaria transmission and in the few areas in which it remains effective. Sulfadoxine-pyrimethamine can be used with artesunate for the treatment of acute uncomplicated malaria in areas where the parasites are still sensitive to sulfadoxine-pyrimethamine.

In malaria-endemic areas in Africa, intermittent preventive treatment with sulfadoxine-pyrimethamine combination is strongly recommended in all women (SP-IPTp) as part of antenatal care. At least three doses of sulfadoxine-pyrimethamine are administered during pregnancy, a single oral dose of three tablets each (one tablet contains $25 \mathrm{mg}$ sulfadoxine and $500 \mathrm{mg}$ pyrimethamine). Dosing should start in the second trimester, and doses should be given at least 1 month apart, with the objective of ensuing that at least three doses are received.
In areas of moderate-to-high malaria transmission of Africa where sulfadoxine-pyrimethamine is still effective (no pfdhps540 mutation), intermittent preventive treatment with this combination (one to two tablets) is recommended to infants (aged lower than 12 months) (SP-IPTi) at the time of the second and third rounds of vaccination against diphtheria, tetanus, and pertussis (DTP) as well as vaccination against measles.

In areas with high malaria transmission in the sub-Sahel region of Africa, seasonal malaria chemoprevention (SMC) with monthly amodiaquinesulfadoxine-pyrimethamine (ACT) combination is strongly recommended for all children aged lower than 6 years during each transmission season.

From an operational perspective, it is noted that drugs used in IPTp, SMC, and IPTi should not be used as a component of first-line treatments in the same country or region.

\section{Adverse Reactions and Toxicity}

Sulfadoxine-pyrimethamine combination is generally well tolerated at the recommended doses. Most adverse reactions are those associated with sulfonamides. These include gastrointestinal disturbances (nausea, vomiting, abdominal pain, and diarrhea), headache, dizziness, skin reactions (photosensitivity, rash, pruritus, and urticaria), and slight hair loss. Erythema multiforme, Stevens-Johnson syndrome, and toxic epidermal necrolysis may also occur at rare frequency. Leucopenia, thrombocytopenia, megaloblastic anemia, hemolytic anemia (probably related to G6PD deficiency), crystalluria, hematuria, oliguria, hepatitis, serum sickness, allergic pericarditis, and pulmonary infiltrates resembling eosinophilic or allergic alveolitis have also been reported.

\section{Contraindications}

Sulfadoxine-pyrimethamine alone or combination therapy with amodiaquine or artesunate is contraindicated in the following conditions: known hypersensitivity to pyrimethamine, sulfonamides, and structurally related compounds; megaloblastic anemia due to folate deficiency; premature or newborn infants in the first 2 months of life; 

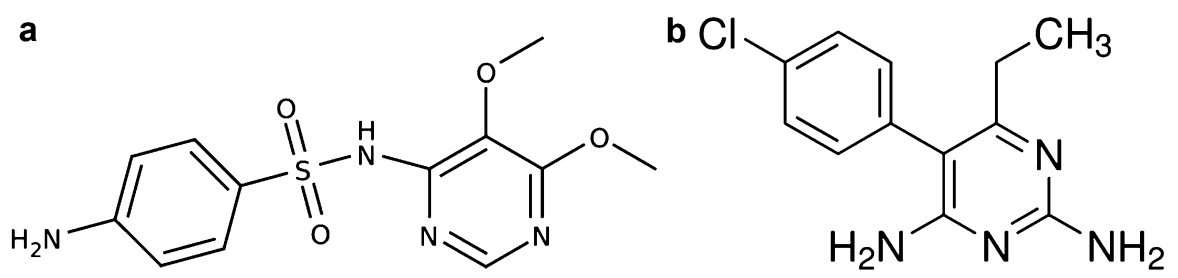

Pharmacology of Antimalarial Drugs, Current Anti-malarials, Fig. 6 Chemical structures of (a) sulfadoxine and (b) pyrimethamine

HIV-infected patients receiving cotrimoxazole prophylaxis against opportunistic infections; and pregnancy in the first trimester.

\section{Caution}

The use of sulfadoxine-pyrimethamine should be discontinued if skin eruption, cytopenia, or a bacterial or fungal superinfection occurs. Repetitive administration of the medication to patients with blood dyscrasia and renal or hepatic failure should be performed with caution as the drugs may accumulate to toxic levels.

\section{Pharmacokinetics}

Pharmacokinetic parameters of sulfadoxine and pyrimethamine following currently recommended doses for the treatment, seasonal chemoprevention, or intermittent preventive treatment of uncomplicated malaria are summarized in Table 1.

Sulfadoxine and pyrimethamine are readily absorbed from the gastrointestinal tract after oral administration. Plasma protein binding is about $90 \%$, mainly to albumin. The $t_{1 / 2}$ of sulfadoxine is longer than pyrimethamine (4-11 days and $60-450 \mathrm{~h}$, respectively). The $\mathrm{Vd} / \mathrm{F}$ of pyrimethamine is about ten times larger than sulfadoxine as it is concentrated in the kidneys, lungs, liver, and spleen. Both sulfadoxine and pyrimethamine cross the placental barrier and pass into breast milk. Both are metabolized by the liver, but enzymes responsible for this biochemical process are not well identified. It is only known that sulfadoxine undergoes varying degrees of acetylation, hydroxylation, and glucuronidation. Renal clearance is the main route of excretion of both drugs.

\section{Factors Associated with Altered Drug Exposure and/or Treatment Response}

Children The pharmacokinetics information of sulfadoxine-pyrimethamine in children with malaria has been limited, although it is recognized that the currently recommended $\mathrm{mg} / \mathrm{kg}$ bw dose of sulfadoxine-pyrimethamine achieves substantially lower plasma drug concentrations in young children than in older patients.

Pregnancy The pharmacokinetics of sulfadoxine and pyrimethamine when administered as a fixeddose combination are altered in pregnant women. Pregnant patients appear to have higher $\mathrm{CL} / \mathrm{F}$ and $\mathrm{Vd} / \mathrm{F}$ of both drugs, resulting in lower AUC and shorter $t_{1 / 2}$ than nonpregnant adults. These changes in pharmacokinetics may be associated with high rate of treatment failure in pregnant women.

Drug Interactions Clinical effectiveness of sulfadoxine-pyrimethamine is reduced when the drug is given with high-dose folic acid ( $>5 \mathrm{mg}$ ). Concurrent treatment with trimethoprimsulfamethoxazole should be avoided due to increased risk of severe cutaneous reactions. Additive hematological toxicity may occur when sulfadoxine-pyrimethamine is given in combination with myelosuppressants such as methotrexate, daunorubicin, and cytarabine.

The $\mathrm{Vd} / \mathrm{F}$ of pyrimethamine is slightly increased when sulfadoxine-pyrimethamine combination is co-administered with artesunate. This interaction is unlikely to be clinically significant as total systemic exposure and plasma concentrations up to day 7 are unaffected. 


\section{Atovaquone-Proguanil}

Atovaquone-proguanil is currently indicated for malaria prophylaxis. The combination may also be used for treatment of uncomplicated malaria in travelers outside malaria-endemic areas. In addition, it is recommended for use in combination with artesunate and primaquine as an alternative treatment of uncomplicated malaria in areas where WHO recommended treatments are not available or not effective. The atovaquone component was initially developed as a potential antimalarial for monotherapy. It was effective agent with a broad-spectrum antiparasitic activity. A search for combination partner with potential synergy with atovaquone identified the folate inhibitor proguanil as a candidate.

\section{Chemistry and Physical Properties}

Atovaquone(2-(trans-4-(P-Chlorophenyl)cyclohexyl)-3-hydroxy-1,4naphthoquinone: Fig. 7a) is a hydroxynaphthoquinone with molecular weight of 366.8. Proguanil ( $N$-(4-Chlorophenyl)- $n$ '-(isopropyl)-imidodicarbonimidic diamide: Fig. 7b), also known as chlorguanide or chloroguanide, is a biguanide compound with molecular weight of 253.7.

\section{Pharmacology}

Antimalarial Activities and Mechanism of Action and Resistance Atovaquone is active against all stages of all Plasmodium species. It is also active against liver stages, resulting in its utility as a prophylactic drug. However, it is not believed to be active against "dormant" hypnozoites. Atovaquone acts as a competitive inhibitor of ubiquinol, specifically inhibiting the mitochondrial electron transport chain at the bc1 complex. Inhibition of bc 1 activity results in a loss of mitochondrial function. Atovaquone exerts potent antimalarial activity with $\mathrm{IC}_{50}(50 \%$ inhibitory concentration) as low as $1-3.5 \mathrm{nM}$.

Proguanil appears to be active against almost all stages of the malaria parasite life cycle, but clinical use is limited to blood stages. In vivo, it is converted into an active triazine metabolite, cycloguanil (Fig. 7c), which acts by inhibiting parasite's dihydrofolate reductase enzyme, similarly to that of pyrimethamine.

The combination of atovaquone and proguanil acts synergistically. The parent compound, proguanil, rather than the metabolite, cycloguanil, has been demonstrated to synergize the antimalarial activity of atovaquone, lowering the effective concentration at which atovaquone collapses the mitochondrial membrane potential. Atovaquoneproguanil is seldom used in endemic areas because of the propensity for emergence of highgrade resistance to atovaquone. It is welldocumented that de novo atovaquone resistance occurs very rapidly. This is due to a missense point mutation at position 268 in the cyt $\mathrm{b}$ gene, exchanging tyrosine for serine (Y268S) or, less frequently, asparagine (Y268N). The position 268 in cytochrome $\mathrm{b}$ is highly conserved across all phyla and is located within the "ef" helix component of the Qo site, which is putatively involved in ubiquinol binding.<smiles>CC(C)NC(=N)NC(=N)Nc1ccc(Cl)cc1</smiles><smiles>CC(C)NC(=N)NC(=N)Nc1ccc(Cl)cc1</smiles><smiles>CC(C)NC(=N)NC(=N)Nc1ccc(Cl)cc1</smiles>

\section{Pharmacology of} Antimalarial Drugs, Current Anti-malarials,

Fig. 7 Chemical structures of (a) atovaquone,

(b) proguanil, and

(c) cycloguanil 
Other Pharmacological Activities Atovaquone is clinically used for the treatment or prevention of Pneumocystis carinii pneumonia (PCP) in patients who are intolerant to trimethoprimsulfamethoxazole combination. In addition, it is also used for the treatment or prevention of toxoplasmosis and babesia.

\section{Therapeutic Indications for Malaria}

Currently, the fixed-dose combination of atovaquone-proguanil is used as a chemoprophylactic agent for preventing malaria in travelers. It may also be considered for the treatment of uncomplicated malaria in travelers outside malaria-endemic areas and for use in combination with artesunate and primaquine as an alternative treatment for uncomplicated malaria, where WHO recommended treatments are not available or not effective.

\section{Adverse Reactions and Toxicity}

Atovaquone-proguanil is generally well tolerated with mild adverse reactions. Common adverse reactions include headache, cough, and gastrointestinal disturbance (nausea, vomiting, diarrhea, and abdominal pain). Dizziness, oral ulceration, blood disorders (neutropenia and anemia), and skin reactions (photosensitivity rash and erythema multiforme) rarely occur. Other adverse reactions which are occasionally reported are elevated levels of liver enzymes, hepatitis, hepatic failure, allergic reactions (anaphylaxis, angioedema, Stevens-Johnson syndrome, and vasculitis), and pancytopenia in patients with severe renal impairment. A significant concern for the antimalarials targeting the parasite bc1 including atovaquone is host mitochondrial toxicity. In animal models, this manifested itself as acute toxicity (presumed to be cardiotoxicity).

\section{Contraindications}

Atovaquone-proguanil is contraindicated in patients with known hypersensitivity reactions to atovaquone or proguanil or structurally related compounds. Due to increased risk of pancytopenia, it is contraindicated for use as malaria prophylaxis in patients with severe renal insufficiency.

\section{Caution}

The doses of atovaquone-proguanil should be selected cautiously in elderly, based on hepatic, renal, or cardiac function, propensity of higher systemic exposure to cycloguanil, and a greater frequency of concomitant disease or concomitant drug therapy.

\section{Pharmacokinetics}

The pharmacokinetic parameters of atovaquone in the currently utilized formulation (Malarone ${ }^{\mathrm{TM}}$ : $250 \mathrm{mg}$ atovaquone $/ 100 \mathrm{mg}$ proguanil) for malaria prophylaxis and treatment are summarized in Table 1.

Atovaquone exhibits dose-limiting absorption with maximum absorption observed using $750 \mathrm{mg}$ tablets. Poor drug solubility is suggested as the cause of this limited absorption. Furthermore, a marked interpatient variability of atovaquone bioavailability is reported (107\%), which is likely due to low drug solubility and the effects of food. The absorption of atovaquone from the gastrointestinal tract is increased by about fourfold when atovaquone is taken with a high-fat meal (two slices of toast with $56 \mathrm{~g}$ of butter). The drug is therefore recommended to be taken with a highfat meal. There is no unexpected accumulation of atovaquone following repeated administration. Proguanil is readily absorbed from the gastrointestinal tract reaching $\mathrm{C}_{\max }$ within approximately $5 \mathrm{~h}$ of administration.

Atovaquone and proguanil are, respectively, $99 \%$ and $75 \%$ bound to plasma proteins with high affinity to albumin. The long $t_{1 / 2}$ of 2-3 days is characterized by the enterohepatic circulation. The low drug clearance rate suggests that atovaquone may also accumulate in tissues, where it is protected from biliary clearance. Elimination is primarily via the liver, with almost undetectable amounts $(0.6 \%)$ of the parent drug being eliminated via the kidneys. More than $90 \%$ of the drug excreted in bile (and feces) is in the parent form.

Proguanil undergoes bioactivation by CYP2C19 to the active metabolite, cycloguanil (Fig. 7c). Cycloguanil inhibits Plasmodial dihydrofolate reductase and influences DNA synthesis. Proguanil is also further inactivated by 
CYP2C19 and to a lesser extent CYP3A4 to 4-chlorophenylbiguanide. The urinary proguanilcycloguanil metabolic ratio serves as a marker for differential CYP2C19 metabolic activity. Renal excretion of proguanil is about 40 and $60 \%$ of the administered dose.

\section{Factors Associated with Altered Drug Exposure and Treatment Response}

Children The $\mathrm{CL} / \mathrm{F}$ values of both atovaquone and proguanil are related to body weight. While most of the pharmacokinetics of proguanil and cycloguanil are comparable in adults and children, the $t_{1 / 2}$ of atovaquone is shorter in children.

Pregnancy The plasma concentrations of atovaquone and proguanil in pregnant women in the second and third trimesters are approximately half of nonpregnant adults (with and without acute malaria) as a result of expansion of $\mathrm{Vd} / \mathrm{F}$ and increase of $\mathrm{CL} / \mathrm{F}$.

Body Weight Based on the population-based pharmacokinetic analysis, the $\mathrm{CL} / \mathrm{F}$ of atovaquone has been shown to be increased in patients with higher body weights $(60 \%$ increase in an $80 \mathrm{~kg}$ compared with a $40 \mathrm{~kg}$ patient). The $\mathrm{CL} / \mathrm{F}$ of atovaquone appears to be higher in oriental $(8.49 \mathrm{~L} / \mathrm{h})$ and Malay $(9.13 \mathrm{~L} / \mathrm{h})$ subjects compared with Caucasian (1-7.6 L/h) subjects.

\section{Genetic Polymorphism in Drug-Metabolizing}

Enzymes The association between CYP2C19 gene polymorphism and variation in proguanil and cycloguanil plasma concentrations has given rise to the hypothesis that patients with poor metabolizer phenotype are at greater risk of treatment failure. The prevalence of the poor metabolizer phenotype varies substantially among ethnics, and two variant alleles, CYP2C19*2 and $C Y P 2 C 19 * 3$, are largely associated with the poor metabolizer phenotype. Studies in African and Asian populations, however, have not revealed an association between proguanil CYP2C19 metabolic status and treatment or prophylaxis responses as well as the incidence of adverse reactions (mainly gastrointestinal). Based upon these observations, it is proposed that the parent compound proguanil may have a significant intrinsic efficacy independent of its main metabolite cycloguanil. Another plausible explanation may be that undefined metabolite other than cycloguanil, through another metabolic pathway, is responsible for the proguanil efficacy in poor metabolizers.

Drug Interactions The pharmacokinetics of atovaquone and proguanil and its metabolite cycloguanil are unchanged when given as atovaquone-proguanil combination. There is in vitro evidence of possible inhibition of CYP3A4 by atovaquone. Plasma/serum concentrations of aceprometazine, alimemazine, chlorproethazine, chlorpromazine, and etoposide can be increased when co-administered with atovaquone. The risk of QTc prolongation can be increased when atovaquone is combined with artemether.

Pharmacokinetic interactions between atovaquone and antiretrovirals have been reported. Efavirenz, lopinavir, ritonavir, and saquinavir (all highly protein-bound drugs) reduced atovaquone plasma concentrations in HIVinfected patients. On the other hand, a recent case study described an HIV-infected female with a marked increase in plasma concentrations of the antiretrovirals etravirine (55\%) and saquinavir (274\%), but not raltegravir following atovaquone-proguanil prophylaxis. Atovaquone has been shown to inhibit the glucuronidation of zidovudine. Co-administration of atovaquone and the nucleoside reverse transcriptase inhibitor (NRTI) zidovudine increased the exposure (33\% increase in AUC) and decreased the oral clearance $(25 \%)$ of zidovudine in $\mathrm{HIV}$-infected patients. Furthermore, patients taking atovaquone showed a trend toward lower zidovudine/glucuronide plasma concentrations ( $6 \%$ decrease in AUC) and a significant decrease in the ratio between zidovudine/glucuronide and plasma concentrations ( $30 \%$ decrease). Atovaquone exposure itself was unchanged. The increased zidovudine plasma concentrations and reduced zidovudine glucuronidation may potentially lead to increased formation of the CYP450-mediated zidovudine 
metabolite 3-amino-3deoxythymidine, which shows a sevenfold higher toxicity in bone marrow cells compared with the parent drug. Caution is advised in patients taking additional drugs with similar toxicity profiles to zidovudine, particularly hematological toxicity.

Plasma concentration of atovaquone is reduced when given concurrently with metoclopramide and tetracycline. Atovaquone exposure (AUC) has been shown to be markedly decreased $(50 \%)$ in patients with Toxoplasma gondii infection concurrently treated with the anti-TB drug rifampicin but to a lesser extent (34\%) with rifabutin. The concomitant administration of atovaquone and rifampicin is therefore not recommended as rifampicin is a potent inducer of $\mathrm{CYP} 2 \mathrm{C} 19$, and it therefore could affect proguanil antimalarial activity.

Atovaquone causes an increase in free warfarin concentrations to super-therapeutic levels. Lack of pharmacokinetic interactions is reported between atovaquone and the anticonvulsant phenytoin.

Proguanil may potentiate warfarin action but may reduce effectiveness of live typhoid vaccine. The conversion of proguanil to cycloguanil is reduced in the presence of estrogen. Proguanil and cycloguanil have been shown to inhibit P-glycoprotein-mediated taxol transport without being substrates for P-glycoprotein. Interactions between these compounds and other substrates of P-glycoprotein would be expected.

\section{Artemisinin and Derivatives and Artemisinin-Based Combination Therapies (ACTs)}

Artemisinin drugs, originated from the Chinese herb qinghao (Chinese wormwood Artemisia annua L.), belong to a unique class of compounds, sesquiterpene lactone endoperoxide, and are new generation of potent antimalarials. Qinghaosu has been used for over 2000 years in Chinese traditional medicine for the treatment of fever. Its antimalarial application was first described in the Handbook of Prescriptions for Emergencies in the middle of the fourth century in China. The herb was used as a tea for treatment of malaria in China for over 1500 years. Artemisinin (quinghaosu) is the principal compound isolated from $A$. annua and is further derivatized to the more active (four- to fivefold) derivatives, i.e., artesunate, $\beta$-arteether, and dihydroartemisinin. Dihydroartemisinin is also an active human plasma metabolite of artesunate and artemether. Artemisinin derivatives have constituted the key antimalarials that play an essential role in the control of malaria since the emergence and widespread of multidrug-resistant $P$. falciparum malaria. Currently, artesunate, artemether, and dihydroartemisinin are in clinical uses for treatment of malaria, either as monotherapy (for severe malaria) or as components of artemisinin-based combination therapies (ACTs) for uncomplicated malaria. ACTs are currently the most powerful strategy to treat malaria and prevent malaria-related deaths. With this successful discovery of artemisinins, Professor Tu was awarded the Nobel Prize in Physiology or Medicine in 2015 for the discovery of this effective antimalarial compound.

\section{Chemistry and Physical Properties}

Artemisinin (Fig. 8) is a sesquiterpene lactone peroxide with a characteristic endoperoxide moiety essential for antimalarial activity. Artemisinin is poorly soluble in water. Artesunate is a watersoluble hemisuccinate ester which can be administered by intravenous and intramuscular injection. It is a colorless needle crystal or white crystalline powder, odorless, and almost tasteless. Artesunate can be dissolved in sodium bicarbonate solution, forming water-soluble sodium salt. Artemether and arteether (Fig. 8) are the methyl and ethylethers of dihydroartemisinin (Fig. 8) which possess physical and chemical properties similar to artemisinin. Both are easily soluble in a variety of organic solvents such as ethanol, acetone, and chloroform. Both ethers have the advantage of being more oil soluble than artemisinin. 


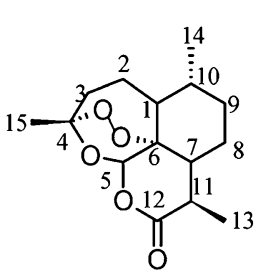

Artemisinin (1)

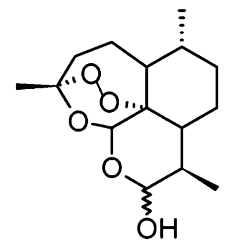

Dihydroartemisinin (2)

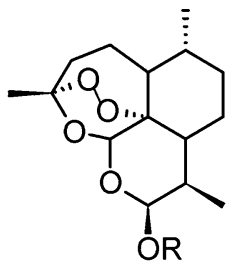

$\mathrm{R}=\mathrm{Me}$ Artemether (3)

Artesunate (5)

\section{$\mathrm{R}=\mathrm{Et} \quad$ Arteether (4)}

Pharmacology of Antimalarial Drugs, Current Anti-malarials, Fig. 8 Chemical structures of artemisinin and derivatives

Artemisinin and its derivatives decompose in protic solvents other than water. Artesunate is more water soluble than other artemisinins and therefore can be administered intravenously. Artemisinin drugs also show a remarkable thermal instability. The derivatives are more unstable than artemisinin. The drugs should be stored in air-tight containers in a cool place below $25^{\circ} \mathrm{C}$ and protected from light. Molecular weights of artemisinin, artemether, $\beta$-arteether, and artesunate are $280,296,312$, and 404 , respectively.

\section{Pharmacology}

Antimalarial Activities and Mechanisms of Action and Resistance

Artemisinin drugs have broad stage specificity against blood stage parasites from the ring stage to early schizonts. They are also active against the early gametocide stages but inactive against extraerythrocytic forms, sporozoites, liver schizonts, and merozoites.

Artemisinin drugs are rapidly acting blood schizontocidal antimalarials against either chloroquine-sensitive or chloroquine-resistant P. falciparum, $P$. vivax, $P$. ovale, and P. malariae. Their potent gametocytocidal activities reduce gametocyte carriage and therefore limit malaria transmission from the treated infection. The artemisinin compounds stop parasite development rapidly, thereby preventing subsequent cytoadherence and rosetting (both are thought to be important pathophysiological mechanisms in severe malaria). Fever and parasites are more rapidly cleared than other antimalarials. Defervescence occurs within 1-2 days, and parasitemia disappears within 2-3 days after drug administration. About $90 \%$ clearance of asexual erythrocytic parasitemia is usually observed within $24 \mathrm{~h}$. Total dose and duration of drug administration have an influence on the cure rate. Correlation between dosage regimen (total dose and duration), severity of the disease, and cure rate has been reported. The longer the duration of treatment, and/or the lesser the severity of the infection, the higher is the cure rate.

Currently, artemisinin monotherapy (artesunate and artemether) is only used for initial treatment of severe malaria but is not recommended for treatment of uncomplicated malaria due to widespread of multidrug-resistant $P$. falciparum and the necessity for prolonged treatment regimens. For this purpose, they are typically used in combination with other structurally unrelated antimalarial drugs as ACTs. ACTs are co-formulations of a fast-acting, highly potent artemisinin and a slowacting, less-potent partner drug (e.g., mefloquine, piperaquine, and lumefantrine) that are given orally over 3 days. During their short half-life, artemisinins leave only residual parasites, which are further eradicated by the slowly eliminated partner drug. The combination is also believed to inhibit the selection of de novo artemisininresistant mutants.

Artemisinin is a sesquiterpene lactone containing a peroxide bridge that plays an essential role in antimalarial effects. The precise molecular targets of action of artemisinins are not well 
understood, but the prevailing theory is the cleavage of the endoperoxide bridge which leads to the formation of reactive carbon radicals that subsequently alkylate essential biomolecules of malaria parasites. A previously proposed molecular mechanism of action for artemisinins is the inhibition of the malarial parasite's calcium ATPase (sarcoplasmic/endoplasmic reticulum calcium ATPase, SERCA). More recent investigations suggest newly identified protein targets in broad pathways involved in the glycolysis, hemoglobin degradation, antioxidant defense, and protein synthesis, processes essential for parasite survival.

The emergence of $P$. falciparum resistance to artemisinin and derivatives has recently developed in the Greater Mekong Subregion (GMS: China, Cambodia, Laos PDR, Myanmar, Thailand, and Vietnam). This worrisome development threatens to make malaria practically untreatable in this region and threatens to compromise global endeavors to eliminate this disease. Initial widespread use of artemisinins as monotherapy in the GMS is likely to be the key factor that contributes to their reduced efficacy and/or resistance reported in recent years along the Thai-Cambodian and Thai-Myanmar borders. Artemisinin resistance is characterized as a delay in parasite clearance time. Partial resistance to artemisinin in P. falciparum was first reported in 2008, in Battambang Province in western Cambodia. It was subsequently confirmed in 2009 , in Pailin Province, the well-known epicenter of antimalarial multidrug resistance. Artemisinin resistance has since been reported elsewhere in western Cambodia, western Thailand, southern Myanmar, Southern Vietnam, and China.

A recent series of clinical, in vitro, genomics, and transcriptomics studies in the GMS has shown that artemisinin resistance manifests as slow parasite clearance in patients and increased survival of early ring-stage parasites in vitro. The molecular mechanism underlying this resistance phenotype is single-nucleotide polymorphisms in the parasite's "K13" gene. The K13 propeller gene is located on chromosome 13 and encodes a kelch protein. The mutation is associated with an upregulated "unfolded protein response" pathway that may antagonize the prooxidant activity of artemisinins and in addition the selection of resistance in $P$. falciparum against the partner drug.

\section{Other Pharmacological Activities}

Apart from antimalarial activities, artemisinin drugs have also been shown to exhibit a wide range of pharmacological actions against viruses, helminthes, fungi, and even a variety of cancer cells. In addition, they also possess antiinflammatory and immunosuppressant activities.

In vitro and in vivo studies demonstrated activities of artemisinin and derivatives against other protozoas (Leishmania spp., Trypanosoma spp., Toxoplasma gondii, Neospora caninum, Eimeria tenella, Acanthamoeba castellanii, Naegleria fowleri, Cryptosporidium parvum, Giardia lamblia, Babesia spp.), helminths (Schistosoma species and Fasciola hepatica), fungi (e.g., Cryptococcus neoformans), and viruses (e.g., human cytomegalovirus).

Artemisinins exert selective cytotoxic effects against a wide range of cancer types both in vitro and in vivo. These effects appear to be mediated by artemisinin-induced changes in multiple signaling pathways, interfering simultaneously with multiple hallmarks of cancer (cell cycle arrest, apoptosis, angiogenesis, and cancer invasion and metastasis). Results from a limited number of clinical trials in some types of cancer also support their anticancer property.

Artemisinins have been evaluated in animal models of autoimmune diseases, allergic disorders, and septic inflammation. Their potent antiinflammatory effects have been attributed to the regulation of both innate and adaptive immunity. Artemisinin family drugs can suppress T-cell activation both in vitro and in vivo. In addition, they have been shown to inhibit Toll-like receptors, Syk tyrosine kinase, phospholipase $\mathrm{C} \gamma, \mathrm{PI} 3 \mathrm{~K} /$ Akt, MAPK, STAT-1/3/5, NF-кB, Sp1, and Nrf2/ARE signaling pathways. Artemether suppresses T-cell proliferation and IL-2 production in response to TCR engagement or mitogens in vitro. 


\section{Therapeutic Implications for Malaria}

\section{Uncomplicated Malaria}

The artemisinins rapidly relieve signs and symptoms of acute malaria by rapidly clearing parasitemia. Parasite numbers is reduced by $100-$ to 1000-fold per asexual cycle of the parasite (a factor of approximately 10,000 in each 48-h asexual cycle). This action is fastest among the currently available antimalarial drugs. Because artemisinin and its derivatives are eliminated rapidly, when given alone, a 7-day course of treatment is required. In 2001, the WHO initially recommended the use of ACTs as first-line treatment of uncomplicated malaria in areas where the parasites were resistant to monotherapy with other drugs including chloroquine, sulfadoxinepyrimethamine, and mefloquine. This recommendation was extended to the entire malaria-endemic areas of the world in 2006, and the use of artemisinin in monotherapy was banned to prevent the selection of artemisinin-resistant parasites. In 2012, five different 3-day ACTs, including dihydroartemisinin-piperaquine, artesunate-mefloquine, artemether-lumefantrine, artesunate-sulfadoxine-pyrimethamine, and artesunate-amodiaquine, were deployed in 79 countries with endemic malaria. The concept of combination therapy relies on the rapid onset of schizonticidal action of artemisinins to rapidly reduce parasitemia, leaving the residual parasitemia to be cleared by high concentrations of the partner drugs. This results in the protection of artemisinin by its partner drug from developing resistance and vice versa. An additional advantage from a public health perspective is the ability of the artemisinins to reduce gametocyte carriage and, therefore, the transmissibility of malaria. This contributes to malaria control, particularly in areas of low-to-moderate endemicity. Shorter courses (1-2 days) are not recommended as they are less effective, have less effect on gametocytes, and provide less protection for the slowly eliminated partner drug.

Currently (since 2015), these five ACTs are currently recommended by the WHO for treatment of uncomplicated malaria in all endemic areas in the following clinical situations: (i) Treatment of children and adults with uncomplicated $P$. falciparum malaria except the first-trimester pregnant women. The ACTs can be used safely in pregnant women during the second and third trimesters.

(ii) Nonimmune travelers with uncomplicated P. falciparum malaria returning to nonendemic settings.

(iii) An alternative treatment to chloroquine in adults and children with uncomplicated $P$. vivax, $P$. ovale, $P$. malariae, or $P$. knowlesi in areas with chloroquinesensitive infections.

(iv) First-line treatment of adults and children with chloroquine-resistant $P$. vivax, as well as $P$. ovale, P. malariae, or P. knowlesi.

The choice of ACTs in a country or region should be based on background of drug resistance (particularly of the partner drugs), optimal efficacy, safety, and patients' compliance. Fixed-dose combinations rather than co-blistered or loose, singleagent formulations are recommended whenever possible. For young children and infants, pediatric formulations, with a preference for solid formulations rather than liquid formulations, are recommended.

Resistance of $P$. falciparum to the currently used ACTs has now emerged and is following a similar pattern of resistance previously observed with other antimalarial drugs. Thus far, studies have documented evidence of $P$. falciparum resistance to artemisinins in five countries of the GMS, i.e., Cambodia, Laos, Myanmar, Thailand, and Vietnam, and this was confirmed on the Cambodia-Thailand border. It is therefore, crucial to monitor the efficacy and safety of newly formulated ACTs in view of artemisinin resistance.

Clinical uses of the currently used ACTs are as follows:

\section{(i) Artemether-lumefantrine}

The combination is available as dispersible or standard tablets containing $20 \mathrm{mg}$ artemether and $120 \mathrm{mg}$ lumefantrine (the flavored dispersible pediatric formulation facilitates use in young 
children) or as standard tablets containing $40 \mathrm{mg}$ artemether and $240 \mathrm{mg}$ lumefantrine in a fixeddose combination formulation. The advantage of this ACT is that the combination partner, lumefantrine, is not available as monotherapy and has never been used alone for malaria treatment. The recommended adult dose regimen is artemether-lumefantrine given twice a day at a total six doses for 3 days (four tablets per dose). The patient should receive the initial dose, followed by the second dose $8 \mathrm{~h}$ later, and then one dose twice daily for the following 2 days. The drug should be taken immediately after food or fat-containing drink (e.g., milk) particularly in the second and third days of treatment.

\section{(ii) Artesunate-amodiaquine}

The combination is available as a fixed-dose combination in tablets containing $25 / 67.5 \mathrm{mg}$, $50 / 135 \mathrm{mg}$, or $100 / 270 \mathrm{mg}$ of artesunateamodiaquine. A total therapeutic dose range of 6-30 $\mathrm{mg} / \mathrm{kg}$ bw per day artesunate and 22.5-45 $\mathrm{mg} / \mathrm{kg}$ bw per day amodiaquine for 3 days is recommended.

\section{(iii) Artesunate-mefloquine}

The combination is available as a fixed-dose formulation of pediatric tablets containing $25 \mathrm{mg}$ artesunate and $55 \mathrm{mg}$ mefloquine hydrochloride (50 mg base) or adult tablets containing $100 \mathrm{mg}$ artesunate and $220 \mathrm{mg}$ mefloquine hydrochloride (200 mg base). A total therapeutic dose of $4(2-10) \mathrm{mg} / \mathrm{kg}$ bw per day artesunate and 8.3 (5-11) $\mathrm{mg} / \mathrm{kg}$ bw per day mefloquine, given once a day for 3 days, is recommended. to reduce vomiting, mefloquine dose should be split over 3 days, as in current fixed-dose combination.

\section{(iv) Dihydroartemisinin-piperaquine}

The combination is available as a fixed-dose combination in tablets containing $40 \mathrm{mg}$ dihydroartemisinin and $320 \mathrm{mg}$ piperaquine and a fixed-dose combination pediatric tablets containing $20 \mathrm{mg}$ dihydroartemisinin and
$160 \mathrm{mg}$ piperaquine. The recommended dose regimen for adults and children weighing $>25 \mathrm{~kg}$ is a total dose of $4(2-10) \mathrm{mg} / \mathrm{kg}$ bw per day dihydroartemisinin and $18(16-27) \mathrm{mg} / \mathrm{kg}$ bw per day piperaquine given once a day for 3 days. In children aged $<25 \mathrm{mg} / \mathrm{kg}$, a minimum of $2.5 \mathrm{mg} / \mathrm{kg}$ body weight per day of dihydroartemisinin and $20 \mathrm{mg} / \mathrm{kg}$ bw per day of piperaquine for 3 days is recommended.

\section{(v) Artesunate-sulfadoxine-pyrimethamine}

The combination is available in blister-packed, scored tablets containing $50 \mathrm{mg}$ artesunate and fixed-dose combination tablets containing $500 \mathrm{mg}$ sulfadoxine $/ 25 \mathrm{mg}$ pyrimethamine. There is no fixed-dose combination. A total therapeutic dose of $4(2-10) \mathrm{mg} / \mathrm{kg}$ bw per day artesunate given once a day for 3 days and a single administration of at least 25/1.25 (25-70/ $1.25-3.5) \mathrm{mg} / \mathrm{kg}$ bw per day sulfadoxinepyrimethamine, given once a single dose on day 1 , are recommended. Clinical use of this ACT should be avoided in uncomplicated P. falciparum malaria patients coinfected with HIV/AIDS if they are being treated with the antiretroviral drugs efavirenz or zidovudine. The disadvantage of this ACT is that it is not available as a fixed-dose combination, which may compromise patients' adherence and increase the risk for distribution of loose artesunate tablets, despite the WHO ban on artesunate monotherapy. Resistance is likely to be increased with continued widespread use of sulfadoxine-pyrimethamine, sulfalenepyrimethamine, and cotrimoxazole (trimethoprimsulfamethoxazole). Its use is restricted to areas where sulfadoxine-pyrimethamine is still active, which excludes all of Southeast Asian countries, most of South American, East African, and West African countries.

\section{(vi) Artesunate-pyronaridine}

Apart from the five WHO recommended ACT is another EMA (European Medicines Agency) recommended, US FDA approved, and WHO pre-qualified and on the list of Essential Pediatric 
Medicines of WHO. The combination is available as film-coated tablets Pyramax $^{\mathrm{TM}}$, developed by Shin Poong Pharmaceutical, China, University of Iowa, USA, and MMV). Each tablet contains $180 \mathrm{mg}$ pyronaridine tetraphosphate and $60 \mathrm{mg}$ artesunate). Artesunate-pyronaridine combination is indicated in the treatment of acute, uncomplicated malaria infection caused by P. falciparum or $P$. vivax in adults and children weighing $20 \mathrm{~kg}$ or more. A total therapeutic doses ranging from one to four tablets given once a day for 3 days are recommended. A granule formulation is available for children weighing between $5 \mathrm{~kg}$ and under $20 \mathrm{~kg}$.

\section{Severe Malaria}

Parenteral artesunate is recommended for treatment of severe malaria. While the possible risk for teratogenicity limits the use of artemisinin drugs in the treatment of uncomplicated malaria in pregnant women in the first trimester, treatment of severe malaria is recommended as it is potentially lifesaving for the mother. Compared to quinine, artemisinins treatment in the first trimester is not associated with an increased risk of miscarriage or stillbirth. No difference in the prevalence of major congenital anomalies is observed, although available data have been limited. The benefits of using artemisinins to treat malaria in early pregnancy are likely to outweigh the adverse outcomes of partially treated malaria.

Artemether did not prove to be better than quinine on survival rate, and artesunate is the first choice in low-transmission areas. Intravenous or intramuscular artesunate at $2.4 \mathrm{mg} / \mathrm{kg}$ bw per dose is strongly recommended in adults and children with severe malaria (including infants, pregnant women in all trimesters, and lactating women) for at least $24 \mathrm{~h}$ until they can tolerate oral medication and then followed by a 3 days ACT (with a single-dose primaquine in areas with low transmission). Alternatively, a simplified three doses of $4 \mathrm{mg} / \mathrm{kg}$ bw intravenous artesunate (given at 0,24 , and $48 \mathrm{~h}$ ) instead of five doses of $2.4 \mathrm{mg} / \mathrm{kg}$ are recommended. In children weighing lower than $20 \mathrm{~kg}$, a higher dose of artesunate $(3 \mathrm{mg} / \mathrm{kg}$ bw per dose) is strongly recommended to ensure equivalent exposure to the drug.

If artesunate is not available, artemether in preference to quinine is recommended for both children and adults with severe malaria. In situations where complete treatment of severe malaria is not possible but injections are available, a single intramuscular dose of artesunate (if not available, intramuscular artemether or quinine) is strongly recommended in adults and children with severe malaria before transferal to an appropriate facility for further care. Where intramuscular artesunate is not available, a single rectal dose of $10 \mathrm{mg} / \mathrm{kg}$ bw of artesunate is strongly recommended in children aged lower 6 years (do not use in older children and adults) before transferal to an appropriate facility for further care.

\section{Adverse Reactions and Toxicity}

Artemisinin drugs are generally well tolerated after both oral- and parenteral-dose administration in humans. Post-artemisinin delayed hemolysis is commonly found with artemisinins when used for treatment of severe malaria. Although no deaths have been reported so far, this serious adverse reaction may lead to life-threatening anemia. Preclinical studies showed considerable toxicity of artemisinin drugs at high doses particularly the central nervous system and liver function abnormalities and reduction in the reticulocyte counts. Most of the effects returned to normal within $48 \mathrm{~h}$. Nevertheless, large clinical studies and metaanalyses do not show serious adverse effects in humans. The inconsistencies of results of the preclinical and clinical studies are explained by the relationship between pharmacodynamics (toxic effects) and plasma concentrations of artemisinin drugs and active metabolite dihydroartemisinin. Most of the observed serious toxicity in experimental animals is acute toxicity associated with long-term rather than acute drug exposure. Rapid elimination of artemisinin drugs after oral-dose administration represents a relatively safe route of administration compared to delayed drug release after intramuscular injection. Furthermore, there are drug-related differences in toxicological profiles of artemisinins. Intramuscular application of artemether or -arteether, but not to artesunate, is 
safe and profiles acceptable plasma concentrationtime profiles following intramuscular administration in severe malaria.

Adverse reactions of artemisinins that may occur in clinical uses include hypersensitivity reactions (estimated risk of 1 in 3000), mild gastrointestinal disturbance, dizziness, reticulocytopenia, and elevated liver enzyme activity. Although no ECG abnormalities have been found in most studies, bradycardia and very slight prolongation of the QTc interval have been reported. While studies in experimental animals show neurotoxicity after parenteral artemether, clinical, neurological, and pathological studies in humans have not shown similar findings.

In experiment animals, dose-dependent fetal toxicity was observed after administration of artesunate in the first trimester and was more likely to occur with increased duration of treatment. However, there is no evidence that artemisinin derivatives are teratogenic in humans, but experience is limited.

Artemether-lumefantrine and dihydroartemisinin-piperaquine are the most intensively studied antimalarial drugs with regard to cardiotoxicity. Artemether-lumefantrine has a wide therapeutic index and is generally well tolerated. Common adverse reactions are nausea, dizziness, and headache, which are not easily distinguishable from symptoms of acute malaria. It does not significantly prolong QTc interval. No sudden death has been attributed to cardiotoxicity following artemetherlumefantrine.

Dihydroartemisinin-piperaquine is well tolerated. Common adverse reactions that may occur include nausea, vomiting, diarrhea, anorexia, anemia, dizziness, headache, sleep disturbance, and cough. Although there was no evidence of cardiotoxicity in large randomized trials and extensive use of dihydroartemisinin-piperaquine, the partner drug piperaquine does prolong the QTc interval on ECG (reflecting ventricular repolarization which may cause potentially lifethreatening ventricular arrhythmia) by approximately the same magnitude as chloroquine but lower than quinine. One possible sudden cardiac death associated with dihydroartemisininpiperaquine was reported among 200,000 patients. Dihydroartemisinin-piperaquine was reported to increase the QTc interval by 45.2, 35.5 , and $21.0 \mathrm{~ms}$ in healthy subjects following the administration of each dose with high (1000 kcal) or low (400 kcal) fat/calorie meal and fasting conditions, respectively. None had a QTc interval greater than $500 \mathrm{~ms}$.

\section{Contraindications}

Artemisinin drugs are contraindicated in patients with known hypersensitivity to any artemisinin derivative. All ACTs should not be administered to patients with known hypersensitivity to artemisinins and combination partners. Dihydroartemisininpiperaquine should not be used in patients with congenital QTc prolongation or who have a clinical condition or are on medication that results in QTc interval prolongation.

\section{Caution}

Artemether should not be given to patients with meningitis as a marked increase in the CSF concentrations may occur. Artemether should also be used with caution in patients with acute renal failure due to possible drug accumulation.

Due to limited information on safety profile in patients aged over than 65 years or children weighing less than $5 \mathrm{~kg}$, clinical use of artemether-lumefantrine should be with caution. Similarly, dihydroartemisinin-piperaquine should be used in patients aged over 70 years, infants weighing lower than $5 \mathrm{~kg}$, and patients with renal or hepatic impairment with special caution in view of the lack of evidence on the safety profiles of the drug in these groups.

Although there is no evidence for iatrogenic toxicity of artemether-lumefantrine in patients with congenital or clinical conditions resulting in QTc prolongation, a family history of congenital long QT syndrome or sudden death, electrolyte abnormalities such as hypokalemia or hypomagnesemia, the drug should not be used in these patients as it may affect cardiac conductivity.

\section{Pharmacokinetics}

The pharmacokinetic parameters of artemether (when given by intramuscular injection for treatment of severe malaria) and artesunate (when 
given by intravenous injection, intramuscular injection, and rectal route for severe malaria, as well as oral route for uncomplicated malaria) together with their active plasma metabolite dihydroartemisinin are summarized in Table 1.

Artesunate (water soluble) is suitable for administration by all routes, while artemether (water insoluble, lipid soluble) can be administered by intramuscular, oral, or rectal (suppository) route. Artemether (lipid soluble) is currently available for clinical use as oral and intramuscular formulations. Oral dihydroartemisinin is currently in clinical use as co-formulation with piperaquine (ACT). The rectal administration has emerged as one of the important routes, especially in tropical countries where it can be lifesaving. Nevertheless, there is large interindividual variation in the drug bioavailability.

Artemisinin and derivatives exhibit unique and highly variable pharmacokinetic properties. All are rapidly absorbed and eliminated. The $t_{1 / 2}$ is short ranging from 0.5 to $4 \mathrm{~h}$. $\mathrm{T}_{\max }$ of artemisinin drugs vary from minutes to hours, depending on the drug formulation and route of administration. Bioavailability of artemisinin drugs is also highly variable $(<25->85 \%)$, depending on the drug formulation, route of administration, health status, and the nature of malaria infection. The bioavailability of oral artesunate and artemether following oral-dose administration is as low as low $30 \%$ due to high first-pass metabolism. Oral artemether peaks in about 2-6 h. Oral dihydroartemisinin is rapidly absorbed from the gastrointestinal tract with marked interindividual variation. $T_{\max }$ is achieved at about $1-2 \mathrm{~h}$.

Following an intravenous injection of artesunate, high initial plasma concentration is observed and is subsequently rapidly declined. Dihydroartemisinin concentration reaches $t_{\max }$ within 9-20 min after artesunate dosing. Intramuscular artesunate produces lower $\mathrm{C}_{\max }$ of artesunate and delayed $\mathrm{t}_{\max }$ of dihydroartemisinin. Following oral administration, $\mathrm{C}_{\max }$ of artesunate and dihydroartemisinin are achieved within 1 and 1-2 h, respectively. Following rectal administration, pharmacokinetics of artesunate and dihydroartemisinin are similar to oral artesunate, except delayed $t_{\max }$ of artesunate. Intramuscular artemether is absorbed slowly and erratically.

Plasma protein binding of artemisinin drugs ranges from $43 \%$ to $95 \%(44-93 \%, 93 \%$, and $95 \%$ for dihydroartemisinin, artesunate, and artemether, respectively). Artesunate and artemether are extensively converted to the active plasma metabolite dihydroartemisinin which accounts for most of antimalarial activity. High concentrations can be found in the bile, liver, and kidney.

Artesunate and artemether are rapidly (within 15 min of dosing) metabolized in the liver to form the active metabolite dihydroartemisinin by CYP2A6, CYP3A4/A5, and CYP3A4 (secondary contribution of CYP2B6 and CYP3A5), respectively. Artesunate is biotransformed into its active plasma metabolite dihydroartemisinin by plasma esterases with possible contribution of CYP2A6. Dihydroartemisinin is rapidly inactivated to dihydroartemisinin- $\beta$-glucuronide by the phase II enzymes UGT1A9 and UGT2B7. The $t_{1 / 2}$ of dihydroartemisinin is approximately $1-2 \mathrm{~h}$. Evidence also suggests rapid elimination of artesunate (in minutes) and artemether (1-11 h). The concentration of artemether parent compound predominates after intramuscular artemether in severe malaria patients.

Several artemisinin drugs are subject to autoinduction of the hepatic first-pass metabolism, resulting in a decline in bioavailability after repeated dosing, which might compromise treatment. Artemether and dihydroartemisinin including artemisinin have been shown to induce CYP3A activity. Artemisinin and -arteether also activate CYP2C19, and artemisinin upregulates CYP2B6. In vitro experiments using primary human hepatocytes and in a human intestinal cell line suggest that activation of the xenosensors pregnane $\mathrm{X}$ receptor (PXR) and constitutive androstane receptor (CAR) is the underlying mechanism on CYP450 induction by artemisinins. Artesunate and artemisinin have been shown not to interact with P-glycoprotein (MDR1)-mediated taxol transport in $\mathrm{Caco}_{2}$ cells or with MRP1 and BCRP in multidrug-resistant cells. Likewise, in rats, P-glycoprotein did not 
contribute to intestinal absorption or inducible pharmacokinetics of artemisinin.

\section{Factors Associated with Altered Drug Exposure and/or Treatment Response}

Variation of artemisinin pharmacokinetics between individuals of up to 50 -fold has been reported in both healthy subjects and malaria patients. This variation, however, does not correlate with clinical outcome; similar parasite and fever clearance rates are observed in all malaria patients regardless of clinical outcome. The partner drugs as components of the ACTs may mask any potential artemisinin drug failures. There is evidence that pregnancy per se, malaria infection, and race/ethnicity can alter the pharmacokinetic properties of the ACT components.

\section{Genetic Polymorphisms of Drug-Metabolizing Enzymes and Transporters}

The genetic basis of such a large interindividual pharmacokinetic variability of artemisinin drugs has not clearly been addressed. Phenotypic consequences of polymorphisms in drug-metabolizing enzymes CYP2B6 and UGT2B7 and transport proteins (particularly P-glycoprotein) on the pharmacokinetics and effectiveness of artemisinin drugs are yet to be determined. This is particularly important in view of high prevalence of $C Y P 2 B 6$ functional polymorphisms in several malariaendemic countries.

\section{Pregnancy}

Pregnancy has been reported to alter the pharmacokinetic properties of artemisinin drugs as well as different components of the ACTs. Plasma $\mathrm{C}_{\max }$ and AUC of oral dihydroartemisinin have been shown to be lower in pregnant women compared with nonpregnant women. Results of a more recent systematic analysis show lower $\mathrm{C}_{\max }$ and exposure (AUC) of dihydroartemisinin after oral administration of artemether, artesunate, and dihydroartemisinin in women in the second and third trimesters of pregnancy. With regard to the ACT combination partners, relatively low day 7 plasma concentrations of lumefantrine are commonly found following artemether-lumefantrine combination, indicating a low drug exposure.
The influence of pregnancy on amodiaquine and piperaquine appears not to be clinically relevant. Sulfadoxine plasma concentration is significantly reduced, and clearance rate is higher in pregnancy. For pyrimethamine and mefloquine, available data regarding pharmacokinetic changes in pregnancy have been limited. Lower concentrations achieved in pregnant women may lead to reduced clinical efficacy and increased morbidity and mortality. Higher failure rates have been shown in pregnant women with both artemether and artesunate. Although the exact mechanism is unknown, these discrepancies may be due to pharmacokinetic changes of both drugs that occur in pregnancy such as increases in the CL and Vd. It is essential that dose-optimization studies of ACTs are performed in pregnant women, in order to maximize the clinical efficacy and tolerability of these regimens.

\section{Metabolic Auto-induction}

Several artemisinin drugs suffer from autoinduction of the hepatic first-pass metabolism, resulting in a decline of bioavailability after repeated doses. The pharmacokinetics of the artemisinin drugs have been shown to exhibit an unusual time dependency during a 7-day oral daily regimen.

\section{Renal Disease}

Pharmacokinetics of artemether is changed in patients with acute with increased $\mathrm{C}_{\max }$, AUC, while decreased $\mathrm{Vd}$ and longer $\mathrm{t}_{1 / 2}$ compared with nonrenal failures. Artemether should be used with caution in patients with acute renal failure due to concern about drug accumulation.

Interaction Between Artemisinins and Other Drugs Artemisinin and derivatives induce CYP2B6 and CYP2C19, CYP3A (3A4/3A5), and CYP2A6 and inhibit CYP1A2. Clinical studies with the most commonly used ACTs, artemether-lumefantrine, artesunate-mefloquine, and artesunate-amodiaquine, have so far not shown any clinical significant interactions. Tafenoquine co-administration (as gametocytocide or tissue schizonticide) had no clinically relevant effects on dihydroartemisinin, piperaquine, artemether, or lumefantrine 
pharmacokinetics. The pharmacokinetics of artemether, dihydroartemisinin, or mefloquine are not significantly changed when oral artemether is given with mefloquine. Artesunate does not influence atovaquone or proguanil pharmacokinetics, and the triple-drug combination of atovaquone, proguanil, and artesunate is well tolerated.

\section{Drug Interactions of ACTs}

Artemether-lumefantrine: Absorption of the hydrophobic lipophilic lumefantrine component of artemether-lumefantrine combination varies widely between individuals and is greatly enhanced with fat co-administration. Decreased exposure to lumefantrine has been documented in young children ( $<3$ years), pregnant women, large adults, smokers, and patients co-administered with mefloquine, rifampicin, or efavirenz. These patients should be closely monitored and their full adherence to medication ensured. On the other hand, increased exposure to lumefantrine has been documented in patients co-administered with lopinavir, ritonavirboosted lopinavir antiretroviral agents, but without increase in toxicity. No dose adjustment is indicated. There is no pharmacokinetic interaction between tafenoquine and artemether-lumefantrine; both can be co-administered safely.

Artesunate-amodiaquine: Treatment failure after amodiaquine monotherapy is more frequent among children who are underweight for their age. Therefore, their response to treatment should be closely monitored. Furthermore, this ACT is associated with severe neutropenia, particularly in patients coinfected with HIV and especially in those on zidovudine and/or cotrimoxazole. Concomitant use of efavirenz increases exposure to amodiaquine and hepatotoxicity. Concomitant use of artesunate-amodiaquine with these drugs should be avoided. No significant changes in the pharmacokinetics of amodiaquine or its metabolite monodesetylamodiaquine have been observed during the second and third trimesters of pregnancy. No dose adjustment is recommended. No effect of age has been observed on the plasma concentrations of amodiaquine and monodesetylamodiaquine. No dose adjustment by age is indicated.
Artesunate-mefloquine: Concomitant use of the anti-TB drug rifampicin with mefloquine may result to a marked decrease in exposure of mefloquine, which may potentially decrease antimalarial efficacy of mefloquine. Patients taking this drug should be followed up carefully to identify treatment failure.

Dihydroartemisinin-piperaquine: Consumption of a high-fat, high-calorie meal markedly increases piperaquine exposure $\left(\mathrm{AUC}_{0-168 \mathrm{~h}}\right)$. High-fat meal significantly accelerates the absorption of piperaquine, thereby increasing the risk for potentially arrhythmogenic delayed ventricular repolarization (prolongation of the QTc interval). It is therefore a general recommendation that piperaquine-dihydroartemisinin should not be administered at least $3 \mathrm{~h}$ before or after food consumption. Malnourished children are at increased risk of treatment failure following treatment with dihydroartemisinin-piperaquine due to decreased plasma concentrations. Their response to treatment should be closely monitored. In pregnant women, dihydroartemisinin and piperaquine exposure are reduced and may increase the risk of treatment failure. In addition, the increase in clearance of piperaquine in pregnancy results in decreased $t_{1 / 2}$. This may shorten the posttreatment prophylactic effect of dihydroartemisinin-piperaquine combination. However, as this pharmacokinetic change does not affect primary efficacy, no dosage adjustment is recommended. There is no pharmacokinetic interaction between tafenoquine and dihydroartemisinin-piperaquine, and both can be co-administered safely.

Artesunate-sulfadoxine-pyrimethamine: The low dose of folic acid ( $0.4 \mathrm{mg}$ daily) that is required to protect the fetuses of pregnant women from neural tube defects does not reduce clinical efficacy of sulfadoxine-pyrimethamine. On the other hand, higher dose (5 mg daily) significantly reduces clinical efficacy of sulfadoxinepyrimethamine, and both drugs should not be given concomitantly.

Interaction Between ACTs and Antiretroviral Drugs Treatment of malaria in patients coinfected with HIV is a major concern due to potential pharmacokinetic as well as pharmacodynamic 
interactions. Several anti-HIV drugs are substrates and/or inhibitors of CYP3A and MDR1 (e.g., protease inhibitors) or CYP2B6 (efavirenz), so there is a potential risk of multiple drug interactions. Artesunate increases plasma concentration of nevirapine. Artemether plasma concentration is increased with ketoconazole but decreased with darunavir/ritonavir, lopinavir/ritonavir, nevirapine, efavirenz, etravirine, and rifampicin.

With regard to the interaction between the ACTs and antiretroviral drugs, the protease inhibitors (PIs) tend to increase the exposure of lumefantrine and decrease the exposure of artemether and its active metabolite dihydroartemisinin when coadministered as artemether-lumefantrine. Coadministration of artemether-lumefantrine with ritonavir-boosted lopinavir (LPV/r) significantly reduces artemether $\mathrm{C}_{\max }$ and AUC but significantly increases lumefantrine exposure. Co-administration of etravirine reduced the AUC of artemether (38\%), dihydroartemisinin (15\%), and lumefantrine (13\%) at steady state. Co-administration of etravirine with artemether-lumefantrine may lower the antimalarial activity of artemether and should therefore be used with caution. Co-administration of artemetherlumefantrine with efavirenz or nevirapine results in a reduction in artemether, dihydroartemisinin, lumefantrine, and nevirapine exposure. Co-administration of darunavir/ritonavir reduced the AUC of artemether (16\%) and dihydroartemisinin (18\%) but increased the AUC of lumefantrine $(28 \%)$ at steady state. Darunavir/ ritonavir can be co-administered with artemetherlumefantrine without dose adjustment but should be used with caution. Co-administration of artemether-lumefantrine has no effect on etravirine, darunavir, or ritonavir AUC. No drug-related serious adverse events are reported during the studies. There has been limited information on the interaction between other ACTs and protease inhibitors. Co-administration of artesunate-mefloquine with ritonavir decreases dihydroartemisinin exposure. Co-administration of artesunate-pyronaridine with ritonavir increases ritonavir exposure. In the presence of ritonavir-boosted lopinavir (LPV/r), artesunate Cmax and systemic exposure (AUC) are significantly increased (45-80\%), while the metabolic ratio of dihydroartemisinin to artesunate is significantly reduced $(72 \%)$. On the other hand, mefloquine $\mathrm{C}_{\max }$ and systemic exposure are significantly reduced (19-37\%). Lopinavir $\mathrm{C}_{\max }$ is significantly reduced $(22 \%)$ but without significant change in AUC. Drug treatments are generally well tolerated with no serious adverse events. The reduction in systemic exposure of all investigated drugs raises concerns of an increased risk of treatment failure rate in coinfected patients and should be further investigated.

Non-nucleoside reverse transcriptase inhibitors (NNRTIs) tend to decrease the exposures of artemether, dihydroartemisinin, and lumefantrine when co-administered with artemetherlumefantrine. However, the exposure of nevirapine is increased. Co-administration of artesunatemefloquine with nevirapine increases artesunate exposure.

\section{Other Currently Used Antimalarial Drugs}

\section{Tetracycline and Derivatives}

Tetracyclines are synthetic antibiotics derived from a cycline naturally produced by Streptomyces bacteria. Doxycycline is a long-acting derivative. Tetracyclines are generally used in the treatment of infections of the respiratory tract and the intestines and are also used in the treatment of chlamydia. Their most common current use is in the treatment of moderately severe acne and rosacea. Doxycycline is also used as a prophylactic treatment for infection by Bacillus anthracis (anthrax) and is effective against Yersinia pestis, the infectious agent of bubonic plague. Tetracyclines remain the treatment of choice for infections caused by chlamydia (trachoma, psittacosis, salpingitis, urethritis, and L. venereum infection), Rickettsia (typhus, Rocky Mountain spotted fever), brucellosis, and spirochetal infections (borreliosis, syphilis, and Lyme disease). In addition, they may be used to treat anthrax, plague, tularemia, and Legionnaires' disease. They may have a role in reducing the duration and severity of cholera, although drug resistance is mounting and their effect on overall mortality is questioned. Tetracycline derivatives 
Pharmacology of

Antimalarial Drugs,

Current Anti-malarials,

Fig. 9 Chemical structures

of tetracycline and

doxycycline a<smiles>CN(C)C1C(O)=C(C(N)=O)C(=O)C2C(O)=C3C(=O)c4c(O)cccc4C(C)(O)C3CC21</smiles><smiles>C[C@@H]1c2cccc(O)c2C(=O)C2=C(O)[C@]3(O)C(=O)C(C(N)=O)=C(O)[C@H](N(C)C)[C@H]3[C@@H](O)[C@@]21O</smiles>

are currently being investigated for the treatment of certain inflammatory disorders.

Tetracycline and doxycycline (Fig. 9a and b) are used for malaria treatment and prophylaxis, as well as treating certain forms of human filariasis. Both have molecular weight of 444.4.

Antimalarial Activities and Therapeutic Indications for Malaria

Tetracycline and doxycycline are broad-spectrum antibiotics which are inhibitors of protein synthetase by disrupting messenger RNA and transfer RNA. Both are consistently active against all species of malaria. Tetracycline and doxycycline are slow-acting blood schizontocidal agents. Currently, doxycycline is used in combination with quinine or artesunate for treatment of chloroquine-resistant and multidrug-resistant P. falciparum. Doxycycline remains effective in combination with quinine or artesunate at a dose of $200 \mathrm{mg}$ for 7 days. In addition, it is also indicated for the prophylaxis of malaria at a dose of $100 \mathrm{mg} /$ day starting at the day of arrival in endemic areas and continuing for up to 4 weeks after returning.

\section{Adverse Drug Reactions and Toxicity}

Common adverse drug reactions of doxycycline include gastrointestinal disturbance (nausea, vomiting, and diarrhea), particularly with higher doses. Oral doxycycline should be administered with food to reduce gastric irritation. Other reactions that can occasionally occur are dry mouth, glossitis, stomatitis, dysphagia, and esophageal ulceration. Esophageal irritation can be reduced by administration of doxycycline with a full glass of water. Doxycycline crosses the placenta and may cause discoloration of teeth and possible bone growth retardation in the fetus and in young infants. They also discolor teeth and cause enamel hypoplasia in young children. However, doxycycline has recently been recommended by the American Association of Pediatrics to be used safely in children of all ages if given less than 28 days. Hypersensitivity reactions such as urticaria, angioneurotic edema, anaphylaxis, anaphylactoid purpura, pericarditis, and exacerbation of systemic lupus erythematosus may occur. Rare severe adverse reactions that can be found include benign intracranial hypertension in adults and hematological abnormalities such as hemolytic anemia, thrombocytopenia, neutropenia, eosinophilia, and thrombophlebitis (with prolonged intravenous administration). Expired tetracyclines or tetracyclines allowed to stand at a pH less than 2 are reported to be nephrotoxic due to the formation of a degradation product, anhydro-4-epitetracycline causing Fanconi syndrome.

\section{Contraindications}

Doxycycline is contraindicated in patients with known hypersensitivity to tetracyclines and related compounds. Doxycycline is categorized by the FDA as a class D drug in pregnancy. Fatal necrosis has been reported with doxycycline use in pregnancy.

As with all tetracyclines, it is contraindicated in pregnancy through infancy, due to the potential for disrupting bone and tooth development. The exception is in the treatment of anthrax or where other medications are contraindicated or ineffective.

\section{Caution}

Doxycycline should be used with caution in patients with gastric or intestinal diseases such as colitis, who may be at greater risk for pseudomembranous colitis. In addition, the use of doxycycline in patients with established systemic lupus 
erythematosus should be with caution as the drug may worsen the clinical conditions.

\section{Pharmacokinetics}

Pharmacokinetic parameters of doxycycline following dose regimens recommended for treatment and prophylaxis of malaria are summarized in Table 1.

There is a marked interindividual variability in doxycycline pharmacokinetics, depending on age and the co-administered drugs. Doxycycline is highly lipophilic and is rapidly and almost completely absorbed after oral administration with bioavailability approaching 100\%. Unlike tetracycline, absorption of doxycycline is not markedly changed in the presence of food. However, the rate and extent of absorption of doxycycline are markedly reduced when taken with milk or other dairy products. Doxycycline should not therefore be administered with these products.

Doxycycline is widely distributed in body fluids and tissues including the bone marrow, breast milk, livers, kidneys, gastrointestinal tract, and spleen. Penetration into sputum is $8-28 \%$ over $16 \mathrm{~h}$. Penetration into saliva is poor, while biliary concentration exceeds serum by manyfold. The drug also crosses the placenta. Approximately $80-95 \%$ of doxycycline binds to plasma proteins. The drug is metabolized in the liver by an unknown mechanism. No metabolites have been identified to date. Tetracyclines including doxycycline undergo enterohepatic recirculation, resulting in slow clearance. Doxycycline is eliminated unchanged by both the renal and biliary routes. Bile concentrations may be 10-25 times those in plasma/serum. Approximately $35-60 \%$ is excreted in urine and the remainder in feces. The $t_{1 / 2}$ of doxycycline is not affected by impaired renal function, renal failure, or hemodialysis. Therefore, dose adjustment is not required for these patients.

\section{Factors Associated with Altered Drug Exposure and/or Treatment Response}

Doxycycline absorption is decreased in the presence of antacids, bismuth subsalicylate, protonpump inhibitors, and oral iron preparations. Hepatic inducers such as rifampicin,

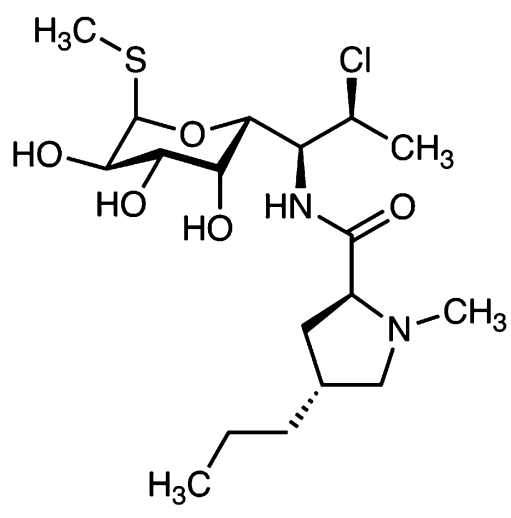

Pharmacology of Antimalarial Drugs, Current Antimalarials, Fig. 10 Chemical structure of clindamycin

anticonvulsants (carbamazepine, phenytoin, phenobarbital), and chronic alcohol use may accelerate doxycycline metabolism resulting in increased drug exposure (AUC) and $\mathrm{C}_{\max }$. The $\mathrm{t}_{1 / 2}$ is also shortened.

\section{Clindamycin}

Antimalarial Activities and Therapeutic Indications for Malaria

Clindamycin (Fig. 10) is a semisynthetic derivative of lincomycin, a natural antibiotic produced by the actinobacterium Streptomyces lincolnensis. It is obtained by $7(S)$-chloro-substitution of the $7(R)$-hydroxyl group of lincomycin.

Clindamycin is used as antibacterial drug, primarily Gram positive and anaerobic infections caused by susceptible anaerobic bacteria, including dental infections and infections of the respiratory tract, skin, and soft tissue, peritonitis, and bone and joint infections. Topical application of clindamycin phosphate is used to treat mild-tomoderate acne.

For treatment of malaria, clindamycin is always used in combination with standard blood schizontocidal drugs particularly quinine and artesunate for treatment of uncomplicated and severe malaria. It is the first-line treatment in first-trimester pregnancies. Clindamycin should not be used alone as an antimalarial drug due to its slow action. 
Its mechanism of action involves inhibition of microbial protein synthesis by preferential binding to the 50S ribosomal subunit and inferences with peptide chain initiation. It is active on apicoplast and leads to delayed cell death.

\section{Adverse Reactions and Toxicity}

Clindamycin is generally well tolerated after oral administration. Adverse reactions include nausea, vomiting, abdominal pain or cramps, rash, or pruritus. High dose (both intravenous and oral) of clindamycin may cause a metallic taste in the mouth. Rarely, clindamycin therapy has been associated with anaphylaxis, blood dyscrasia (leukopenia, agranulocytosis, eosinophilia, and thrombocytopenia), erythema multiforme, polyarthritis, jaundice, raised liver enzymes, hepatotoxicity, and renal dysfunction. Some parenteral formulations contain benzyl alcohol, which may cause fatal "gasping syndrome" in neonates.

\section{Contraindications}

Clindamycin is contraindicated in patients with known hypersensitivity to clindamycin and structurally related compounds.

\section{Caution}

Clindamycin should be used with caution in patients with gastrointestinal diseases (increased risk of pseudomembranous colitis) and severely ill elderly patients (increased risk of diarrhea). Close monitoring of adverse reactions is required when clindamycin is administered to neonates (risk of toxic plasma drug concentrations). Dose optimization may be required when clindamycin is given to patients with moderate-to-severe liver disease due to impairment of drug clearance. Patients with HIV infection should be closely monitored if clindamycin is to be given due to possibility of increased plasma drug exposure to toxic level.

\section{Pharmacokinetics}

Pharmacokinetic parameters of clindamycin are summarized in Table 1.

Clindamycin is rapidly absorbed after oral administration, with an oral bioavailability of approximately $90 \%$. About $90 \%$ is bound to plasma proteins and accumulates in leukocytes, macrophages, and bile. It is widely distributed throughout the body fluids and tissues, including the bone, but significant levels are reached in CSF.

Clindamycin is metabolized in the liver by CYP3A4 into the active $N$-demethyl and sulfoxide metabolites and some inactive metabolites. About $10 \%$ of a dose is excreted in the urine as active drug or metabolites and about $4 \%$ in feces. The remainder is excreted as inactive metabolites. Excretion is slow and takes place over several days. The drug is not effectively removed from the body by dialysis.

Clindamycin $t_{1 / 2}$ may be prolonged and $C L$ reduced in neonates and patients with renal impairment. However, dose modification is not considered necessary.

There has been no data published on the pharmacokinetics of clindamycin in pregnant women, although it is used to treat malaria in pregnancy.

\section{Factors Associated with Altered Drug Exposure and/or Treatment Response}

Clindamycin absorption is delayed with aluminum salts and kaolin. It is a weak-to-moderate inhibitor of CYP3A in vitro, and therefore potential for drug-drug interactions involving clindamycin is low. Nevertheless, the use in patients HIV infection should be with caution. Systemic drug exposure and plasma protein binding of clindamycin have been shown to be increased, while $\mathrm{CL} / \mathrm{F}$ and $\mathrm{Vd} / \mathrm{F}$ reduced in HIVinfected patients.

Clindamycin prolongs effects of neuromuscular blocking agents and may lead to respiratory depression. Possible antagonism and crossresistance may occur with macrolides and chloramphenicol. Clindamycin antagonizes parasympathomimetics such as neostigmine.

\section{Lumefantrine}

Antimalarial Activities and Therapeutic Indications for Malaria

Lumefantrine (benflumetol: Fig. 11) is a fluorine derivative belonging to the arylamino alcohol group of antimalarials structurally related to quinine, mefloquine, and halofantrine. Mechanism of 


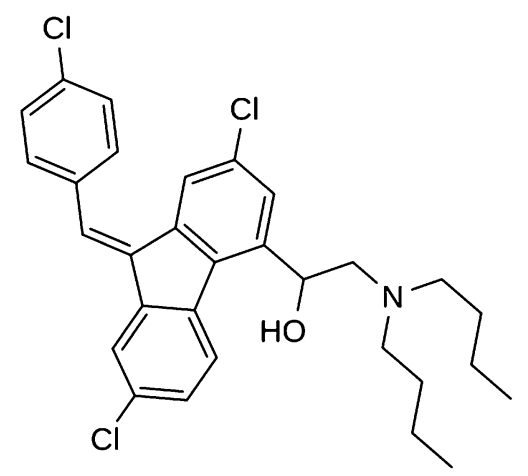

Pharmacology of Antimalarial Drugs, Current Antimalarials, Fig. 11 Chemical structure of lumefantrine

action is thought to be similar to the others in the group by preventing heme detoxification within the parasite food vacuole, thus causing accumulation of the toxic heme complex. It is effective against multidrug-resistant $P$. falciparum malaria. Combination of lumefantrine and artemether (ACT) has proved to be effective in treating uncomplicated acute falciparum malaria. The drug is not available as a single drug and has not been used as monotherapy, which should slow the selection and spread of resistance to this drug.

\section{Pharmacokinetics}

Pharmacokinetic parameters of lumefantrine as a component of artemether-lumefantrine combination (ACT) following dose regimen recommended for treatment and prophylaxis of malaria are summarized in Table 1.

Oral absorption of lumefantrine is increased when co-administered with fatty foods or milk due to its highly lipophilic nature. This may lead to a marked variation in drug absorption among individuals. Its bioavailability and $t_{\max }$ vary within and between individuals, primarily due to fat-dependent absorption. Furthermore, there is a nonlinear absorption of lumefantrine which is saturated at the currently recommended doses. Plasma protein binding is high (99.7\%). Lumefantrine is extensively metabolized in the liver, primarily by the CYP3A4, to the active metabolite desbutyllumefantrine. $\mathrm{T}_{1 / 2}$ is about 3 days.
Factors Associated with Altered Drug Exposure and/or Treatment Response

Plasma lumefantrine concentrations achieved in children aged lower than 5 years are significantly lower than in older children and adults. This has been reported to be associated with increased risks of recrudescence and earlier reinfections. Lumefantrine disposition is also altered in women in the second and third trimesters of pregnancy. Due to a significant increase in $\mathrm{Vd} / \mathrm{F}$, systemic drug exposure is markedly lower. The lower day 7 concentrations reported could be an important factor in clinical failure.

Lumefantrine and amodiaquine are metabolized by CYP3A4 and CYP2C8, respectively, and plasma concentration may be theoretically decreased by the inducers of these enzymes including rifampicin.

\section{Piperaquine}

Antimalarial Activities and Therapeutic Indications for Malaria

Piperaquine (Fig. 12) is a bisquinoline antimalarial drug structurally related to chloroquine and other 4-aminoquinolines. The drug was synthesized independently in France and China in the 1960s. Piperaquine replaced chloroquine for malaria prophylaxis in China in 1978. Nevertheless, with the emergence of $P$. falciparum piperaquine-resistant strains, piperaquine use was decreased in the late 1980s. In the 1990s, piperaquine was reconsidered as one of the components of short-course ACTs in combination with dihydroartemisinin, trimethoprim, and primaquine (China-Vietnam 4 or CV4 and China-Vietnam 8 or CV8).

Piperaquine has a very long $t_{1 / 2}$ of about 2-3 weeks and consequently could be expected to provide a long period of posttreatment prophylaxis.

Both the mechanisms of action and of resistance of piperaquine have not been well characterized but are likely to be similar to chloroquine. Mutation of pfcrt ( $P$. falciparum chloroquine-resistant transporter) as well as increase in plasmepsin II/III copy number has been implicated in piperaquine-resistant P. falciparum. Cross-resistance between piperaquine and chloroquine has been reported. 
<smiles>Clc1ccc2c(N3CCN(CCCN4CCN(c5ccnc6cc(Cl)ccc56)CC4)CC3)ccnc2c1</smiles>

Pharmacology of Antimalarial Drugs, Current Antimalarials, Fig. 12 Chemical structure of piperaquine

However, piperaquine has also been shown to be active against highly chloroquine-resistant P. falciparum. Resistance to both artemisinin and piperaquine is now prevalent in Cambodia, as demonstrated by the increase in the number of cases of dihydroartemisinin-piperaquine treatment failure.

\section{Adverse Reactions and Toxicity}

The symptomatic adverse reactions produced by piperaquine are more or less tolerable. These include dizziness, headache, nausea, vomiting, anorexia, myalgia, cough, asthenia, arthralgia, abdominal distress, pyrexia, eosinophilia, and QTc prolongation.

\section{Pharmacokinetics}

Piperaquine pharmacokinetic properties are similar to those of chloroquine. The kinetics of piperaquine in adult patients is described by a threecompartment model with first-order elimination and two first-order absorption processes. Piperaquine is highly lipid soluble, and its oral bioavailability may be lower when given without any food. A large quantity of co-administered fat enhances absorption significantly.

Piperaquine is highly bound to plasma proteins (>98\%), with a very large $\mathrm{Vd} / \mathrm{F}(>100 \mathrm{l} / \mathrm{kg})$, a low CL/F (1-2 1/h/kg), and a consequently long $\mathrm{t}_{1 / 2}$ (13-28 days).

\section{Factors Associated with Altered Drug Exposure and/or Treatment Response}

The pharmacokinetic properties of piperaquine are affected by body weight and age. Concentrations of piperaquine, when co-administered with dihydroartemisinin, are lower at the beginning of the terminal elimination phase in children than in adults. This low plasma piperaquine concentration on day 7 has been shown to be associated with treatment failure. There are no clinically important differences in the pharmacokinetics of dihydroartemisinin or piperaquine between pregnant and nonpregnant women.

Plasma concentration of piperaquine and potential toxicity are increased when piperaquine is given with verapamil, indinavir, lopinavir/ritonavir, HMG-CoA reductase inhibitors (statins), and cyclosporine. Co-administration of piperaquine with barbiturates, chronic alcohol use, rifampicin, efavirenz, nevirapine, phenytoin, or carbamazepine increases metabolism of piperaquine and therefore reduces plasma concentration of piperaquine with reduced effectiveness.

Co-administration with drugs that prolong QTc interval increases piperaquine risk of cardiotoxicity.

\section{Pyronaridine}

Antimalarial Activities and Therapeutic Indications for Malaria

Pyronaridine is (Fig. 13), a Mannich base 1-aza-acridine structurally related to mepacrine. It is a benzonaphthyridine derivative which was first synthesized in 1970 at the Institute of Chinese Parasitic Disease, Chinese Academy of Preventive Medicine. It was used in China for the treatment of malaria as a single agent for the past 30 years. Pyronaridine-artesunate has been developed by the Medicines for Malaria Venture (MMV) and Shin Poong Pharm Co Ltd (Republic of Korea) for the treatment of acute uncomplicated $P$. falciparum and blood stage $P$. vivax malaria with a fixed-dose combination tablet and granule formulation for pediatric administration (as a potential ACT). It is on the World Health Organization's List of Essential Medicines, the most effective and safe medicines needed in a health system.

Pyronaridine exhibits high efficacy, including against chloroquine- and amodiaquine-resistant strains, and the reassurance of many years of successful use in China as monotherapy and in combination with other antimalarials, without the 
<smiles>COc1ccc2nc3cc(Cl)ccc3c(Nc3cc(CN4CCCC4)c(O)c(CN4CCCC4)c3)c2n1</smiles>

Pharmacology of Antimalarial Drugs, Current Antimalarials, Fig. 13 Chemical structure of pyronaridine

development of widespread drug resistance. Pyronaridine has potent in vitro activity against P. falciparum strains and clinical isolates including those that are resistant to other antimalarials. In vivo animal models indicate a synergistic effect between pyronaridine and artemisinins against parasites resistant to one or both components, restoring efficacy against these strains. Consequently, pyronaridine represents an ideal candidate for combination therapy with artemisinin derivatives, such as artesunate.

Almost all published clinical trials to date used the Chinese enteric-coated tablet formulation with $175 \mathrm{mg}$ of the tetraphosphate, equal to $100 \mathrm{mg}$ base, with dosages lipophilic at $\mathrm{pH} 7.4(\log \mathrm{D}$ 0.34 ); lipophilicity was reduced at $\mathrm{pH} 5$. The base is more liposoluble than the salt. Clinical studies of the combination of pyronaridine tetraphosphate and artesunate are encouraging and show it to be a promising new artemisinin combination therapy for the treatment of both $P$. falciparum and $P$. vivax malaria in adult, children, and infant populations. The drug is administered as pyronaridine tetraphosphate (56.89\% base), a yellow, odorless powder with a bitter taste.

The mechanism(s) by which pyronaridine acts as an antimalarial appears to be similar to chloroquine through inhibition of the production of, and formation of complexes with, $\beta$-hematin to enhance hematin-induced human blood cell lysis. Pyronaridine also inhibits glutathionedependent heme degradation. The mechanism by which resistance to pyronaridine develops is unknown but may be due to a direct effect on the pyronaridine mechanism of action.

\section{Adverse Reactions and Toxicity}

Pyronaridine also appears to be well tolerated in clinical studies. Overall, the acute and subacute toxicity of pyronaridine is generally less than that of chloroquine in all animal species tested. Adverse reactions are mild and are usually resolved within 2 days of starting therapy. The most common adverse events after oral pyronaridine therapy in many cases are similar to the symptoms of malaria, i.e., dizziness, nausea, vomiting, and abdominal discomfort. The incidence of cardiovascular toxicity is also less than that of chloroquine. There are no unexpected findings that would be a particular cause for concern at therapeutic levels in human subjects. The concern of liver toxicity which led to a restriction of pyronaridine use has now been resolved. However, evidence of embryotoxicity in rodents suggests that pyronaridine should be used with caution during pregnancy. There are also some reports of palpitations and allergic skin reaction. Similarly to chloroquine, pruritus may also rarely occur in African patients.

\section{Pharmacokinetics}

Oral bioavailability of pyronaridine is relatively low. The relative bioavailability of enteric-coated tablets and capsules are about $20 \%$ and $32 \%$, respectively. In vitro studies using human CYP450 isoforms indicated that pyronaridine could be metabolized by CYP1A2, CYP2D6, and CYP3A4. Quinoneimine metabolites of aminoquinolines are thought to cause the toxicity problems. Pyronaridine preferentially associates with blood cells with a blood/plasma distribution of 1.2-1.7 in human. Plasma protein binding is high (92-96\%). As pyronaridine concentrates in erythrocytes, plasma assays may underestimate pyronaridine concentrations. The drug is extensively distributed to various body tissues. The $\mathrm{t}_{1 / 2}$ of pyronaridine in healthy adult subjects and adult malaria subjects are estimated to be 11.3 and 13.2 days, respectively. The pharmacokinetics of pyronaridine appears to be altered in patients with 
malaria. In children, the $t_{1 / 2}$ appears to be shorter than adults ranging from 6.6 to 9.0 days.

\section{References}

Abdulla S, Sagara I, Borrmann S, D'Alessandro U, Gonzalez R, Hamel M, et al. Efficacy and sagety of artemether-lumefantrine dispersible tablets compared with crushed commercial tablets in African infants and children with uncomplicated malaria: a randomized, single-blind, multicentre trial. Lancet. 2008;372:1819-27.

Achan JT, Erhart A, Yeka A, Tibenderana JK, Baliraine FN, Rosenthal PJ, et al. Quinine, an old anti-malarial drug in a modern world: role in the treatment of malaria. Malar J. 2011;10:144.

Achari RG, Baldridge JL, Koziol TR. Rapid determination of quinidine in human plasma by High-Performance Liquid Chromatography. J Chromatogr Sci. 1978;16(6):271-3.

Achumba JI, Ette EI, Thomas WO, Essien EE. Chloroquine-induced acute dystonic reactions in the presence of metronidazole. Drug Intell Clin Pharm. 1988;22:308-10.

Adam I, Ali DA, Alwaseila A, Kheir MM, Elbashir MI. Mefloquine in the treatment of falciparum malaria during pregnancy in eastern Sudan. Saudi Med J. 2004;25:1400-2.

Adam I, Tarning J, Lindegardh N, Mahgoub H, McGready R, Nosten F. Pharmacokinetics of piperaquine in pregnant women in Sudan with uncomplicated Plasmodium falciparum malaria. Am J Trop Med Hyg. 2012;87:35-40.

Adelusi SA, Salako LA. Improved fluorometric assay of chloroquine in biological samples. Aust J Pharm. 1980;32:711.

Adelusi SA, Dawodu AH, Salako LA. Kinetics of the uptake and elimination of chloroquine in children with malaria. Br J Clin Pharmacol. 1982;14(4):483-7.

Aderounmu AF, Salako LA, Lindstrm B, Walker O, Ekman L. Comparison of the pharmacokinetics of chloroquine after single intravenous and intramuscular administration in healthy Africans. Br J Clin Pharmacol. 1986;22(5):559-64.

Adijei GO, Kristensen K, Goka BQ, Hoegberg LC, ALifrangis M, Rodrigues OP, et al. Effect of concomitant artesunate administration and cytochrome P4502C8 polymorphisms on the pharmacokinetics of amodiaquine in Ghanaian children with uncomplicated malaria. Antimicrob Agents Chemother. 2008;52:4400-6.

Adjei GO, Adabayeri VM, Annobil SH. Reversible binocular visual loss in temporal association with artesunateamodiaquine treatment in a child on mefloquine chemoprophylaxis. Ghana Med J. 2012a;46:171-3.

Adjei GO, Oduro-Boatey C, Rodrigues OP, Hoegberg LC, Alifrangis M, Kurtzhals JA, et al. Electrocardiographic study in Ghanaian children with uncomplicated malaria, treated with artesunate-amodiaquine or artemether-lumefantrine. Malar J. 2012b;11:420.

Adjepon-Yamoah KK, Woolhouse HM, Prescott LF. The effect of chloroquine on paracetamol disposition and kinetics. Br J Clin Pharmacol. 1986;21:322-4.

Agarwal S, Gupta UR, Gupta RC, Anand N, Agarwal SS. Susceptibility of glucose-6-phosphate dehydrogenase deficient red cells to primaquine enantiomers and two putative metabolite. Biochem Pharmacol. 1988;37(24):4605-9.

Agrawal S, Moser KA, Morton L, Cummings MP, Parihar A, Dwivedi A, Shetty AC, Drabek EF, Jacob CG, Henrich PP, Parobek CM, Jongsakul K, Huy R, Spring MD, Lanteri CA, Chaorattanakawee S, Lon C, Fukuda MM, Saunders DL, Fidock DA, Lin JT, Juliano JJ, Plowe CV, Silva JC, Takala-Harrison S. Association of a novel mutation in the Plasmodium falciparum chloroquine resistance transporter with decreased piperaquine sensitivity. J Infect Dis. 2017;216(4):468-76.

Akintonwa A, Gbajumo SA, Mabade AF. Placental and milk transfer of chloroquine in humans. Ther Drug Monit. 1988;10(2):147-9.

Albert KS, DeSante KA, Welch RD, DiSanto AR. Pharmacokinetic evaluation of a drug interaction between kaolin-pectin and clindamycin. J Pharm Sci. 1978;67:1579-82.

Alcantara AK, Uylangca CV, Sangalang PP, Cross JH. A comparative clinical study of mefloquine and chloroquine in the treatment of vivax malaria. Southeast Asian J Trop Med Public Health. 1985;16(4):534-8.

Ali HM. Reduced ampicillin bioavailability following oral coadministration with chloroquine. J Antimicrob Chemother. 1985;15:781-4.

Aliving AS, Johnson CF, Tarlov AR, Brewer GJ, Kellermeyer RW, Carson PE. Investigation of the haemolytic effect of primaquine and enhancement of its action against exoerythrocytic forms of the Chesson strain of Plasmodium vivax by intermittent regimens of drug administration. Bull World Health Organ. 1960;22:621-31.

Allahyari R, Strother A, Fraser IM, Verbiscar AJ. Synthesis of certain drug primaquine and their in vitro methemoglobin-producing and glutathione-depleting activity in human erythrocytes. J Med Chem. 1984;27(3):407-10.

Alvan G, Ekman L, Lindstrom B. Determination of chloroquine and its desethyl metabolite in plasma, red blood cells and urine by liquid chromatography. J Chromatogr. 1982;229:241-7.

Amold PJ, Stetten OV. High performance liquid chromate graphic analysis of mefloquine and its main metabolite by direct plasma injection with percolumn enrichment and column switching techniques. J Chromatogr. 1986;353:193-200.

Anderson BJ, Holford NH. Understanding dosing: children are small adults, neonates are immature children. Arch Dis Child. 2013;98:737-44.

Annerberg A, Lwin KM, Lindegardh N, Khrutsawadchai S, Ashley E, Day NP, et al. A small 
amount of fat does not affect piperaquine exposure in patients with malaria. Antimicrob Agents Chemother. 2011;55:3971-6.

Armstrong G, Beg MF, Scahill S. Warfarin potentiated by proguanil. Br Med J. 1991;303:789.

Arnold J, Alving AS, Clayman CB. Induced primaquine resistance in vivax malaria. Trans $\mathrm{R}$ Soc Trop Med Hyg. 1961;55:345-50.

Arnold K, Hien TT, Chinh NT, Phu NH, Mai PP. A randomized customized comparative study of artemisinin (qinghaosu) suppositories and oral quinine in acute falciparum malaria. Trans R Soc Trop Med Hyg. 1990;84:499-502.

Ashley EA, Stepniewska K, Lindegardh N, McGready R, Hutagalung R, Hae R, et al. Population pharmacokinetic assessment of a new regimen of mefloquine used in combination treatment of uncomplicated falciparum malaria. Antimicrob Agents Chemother. 2006;50:2281-5.

Ashley EA, Stepniewska K, Lindegardh N, Annerberg A, Kham A, Brockman A, et al. How much fat is necessary to optimize lumefantrine oral bioavailability? Tropical Med Int Health. 2007a;12:195-200.

Ashley EA, Stepniewska K, Lindegardh N, McGready R, Annerberg A, Hutagalung R, et al. Pharmacokinetic study of artemether-lumefantrine given once daily for the treatment of uncomplicated multidrug-resistant falciparum malaria. Tropical Med Int Health. 2007b;12:201-8.

Ashley EA, Recht J, White NJ. Primaquine; the risks and the benefits. Malar J. 2014;13:418.

Auprayoon P, Sukontason K, Na-Bangchang K, Banmairuroi V, Molunto P, Karbwang J. Pharmacokinetics of quinine in chronic liver disease. Br J Clin Pharmacol. 1995;40:494-7.

Babalola CP, Bolaji OO, Ogunbona FA, Sowunmi A, Walker O. Pharmacokinetics of quinine in African patients with acute falciparum malaria. Pharm World Sci. 1998;20:118-22.

Back DJ, Tjia JF. Single dose primaquine has no effect on paracetamol clearance. Eur J Clin Pharmacol. 1987;32:203-5.

Back DJ, Tjia JF. Inhibition of tolbutamide metabolism by substituted imidazole drugs in vivo: evidence for a structure-activity relationship. Br J Clin Pharmacol. 1988;85:121-6.

Back DJ, Purba HS, Park BK, Ward SA, Orme ML'E. Effect of chloroquine and primaquine and primaquine on antipyrine metabolism. Br J Clin Pharmacol. 1983a;16:497-502.

Back DJ, Purba HS, Park BK, Ward SA, Orme ML'E. Effect of chloroquine and primaquine on antipyrine metabolism. Br J Clin Pharmacol. 1983b;16:497-502.

Back DJ, Purba HS, Staiger C, Orme ML'E, Brekenridge AM. Inhibition of drug metabolism by the antimalarials chloroquine and primaquine in the rats. Biochem Pharmacol. 1983c;32(2):257-63.

Back DJ, Purba HS, Stsiger C, Orme ML'E, Breckenridge AM. Inhibition of drug metabolism by the antimalarial drugs chloroquine and primaquine in the rats. Biochem Pharmacol. 1983d;32(2):257-63.

Back DJ, Sutcliffe F, Tjia JF. Tolbutamide as a model drug for the study of enzyme induction and enzyme inhibition in the rat. Br J Clin Pharmacol. 1984a;81:557-62.

Back DJ, Sutcliffle F, Tjia JF. Tolbutamide as a model drug for study of enzyme induction and enzyme inhibition in the rat. Br J Clin Pharmacol. 1984b;81:557-62.

Back DJ, Grimmer SF, Orme ML, Proudlove C, Mann RD, Breckenridge AM. Evaluation of Committee on Safety of Medicines yellow card reports on oral contraceptive-drug interactions with anticonvulsants and antibiotics. Br J Clin Pharmacol. 1988a;25:527-32.

Back DJ, Tjia JF, Karbwang J, Colbert J. In vitro inhibition studies of tolbutamide hydroxylase activity of human liver microsomes by azoles, sulphonamides and quinolones. Br J Clin Pharmacol. 1988b;26:23-9.

Baehner RL, Nathan DG, Castle WB. Oxidant injury of Caucasian G6PD-deficient red blood cells by phagocytosing leukocytes during infection. J Clin Invest. 1971;50:2466.

Baggish AL, Hill DR. Antiparasitic agent atovaquone. Antimicrob Agents Chemother. 2002;46:1163-73.

Baird KJ, Mc Cormick GL, Canfield DJ. Effects of nine putative metabolites of primaquine on activity of the hexose monophosphate shunt in intact human red blood cells in vitro. Biochem Pharmacol. 1986;35:1099-106.

Baire JK, Baski H, Purnomo Bangs MJ, Subianto B, Patchen C, Haffman SL. Resistance to chloroquine by Plasmodium vivax in Irian Jaya, Indonesia. Am J Trop Med Hyg. 1991;44:547-52.

Baker JK, MaChesney JD, Hufford CD, Clark AM. Highperformance liquid chromatographic analysis of the metabolism of primaquine and the identification of a new mammalian metabolite. J Chromatogr. 1982;230:69-77.

Baker JK, McChesney JD, Jorge A. A simple colorimetric method for the determination of primaquine metabolites in urine. Bull World Health Organ. 1985;63:887-91.

Barnes KI, Mwenechanya J, Tembo M, McIlleron H, Folb PI, Ribeiro I, et al. Efficacy of rectal artesunate compared with parenteral quinine in initial treatment of moderately severe malaria in African children and adults: a randomized study. Lancet. 2004;363:1598-605.

Basco LK, Ringwald P. In vitro activities of piperaquine and other 4-aminoquinolines against clinical isolates of Plasmodium falciparum in Cameroon. Antimicrob Agents Chemother. 2003;47:1391-4.

Basco LK, Cillotin C, Gimenez F, Farinotti R, Le Bras J. Absence of antimalarial activity or interaction with mefloquine enantiomers in vitro of the main human metabolite of mefloquine. Trans R Soc Trop Med Hyg. 1991;85:208-9.

Basra A, Mombo-Ngoma G, Melser MC, Diop DA, Würbel H, Mackanga JR, Fürstenau M, Zoleko RM, Adegnika AA, Gonzalez R, Menendez C, Kremsner PG, Ramharter M. Efficacy of mefloquine intermittent 
preventive treatment in pregnancy against Schistosoma haematobium infection in Gabon: a nested randomized controlled assessor-blinded clinical trial. Clin Infect Dis. 2013;56(6):68-75.

Bassat Q, Mulenga M, Tinto H, Piola P, Borrmann S, Menendez C, et al. Dihydroartemisinin-piperaquine and artemether-lumefantrine for treating uncomplicated malaria in African children: a randomised, noninferiority trial. PLoS One. 2009;4:e7871.

Bassi PU, Osakwe A, lash A, Suku C, Kalat M, Jalo I, et al. Safety of artemisinin-based combination therapies in Nigeria: a cohort event monitoring study. Drug Saf. 2013;36(9):747-56. Epub 2013/04/18.

Bateman CN. Quinine toxicity. Adverse Drug React Acute Poisoning Rev. 1986;4:215-33.

Batty KT, Le AT, Ilett KF, Nguyen PT, Powell SM, Nguyen $\mathrm{CH}$, et al. A pharmacokinetic and pharmacodynamic study of artesunate for vivax malaria. Am J Trop Med Hyg. 1998a;59:823-7.

Batty KT, Thu LT, Davis TM, Ilett KF, Mai TX, Hung NC, et al. A pharmacokinetic and pharmacodynamic study of intravenous vs oral artesunate in uncomplicated falciparum malaria. $\mathrm{Br} \mathrm{J}$ Clin Pharmacol. 1998b;45:123-9.

Baty JD, Evans DAP, Robinson PA. The identification of 6-methoxy-8-aminoquinoline as a metabolite of primaquine in man. Biomed Mass Spectrom. 1975;2:304-6.

Baxter K, Prston CL. Stockley's drug interactions. 10th ed. London: Pharmaceutical Press; 2013. ISBN 9780857110619.

Beedham C, Al-Tayib U, Smith JA. Role of Guinea pig and rabbit hepatic aldehyde oxides in oxidative in vitro metabolism of cinchona antimalarials. Am Soc Pharmacol Exp Ther. 1992;20(6):889-95.

Bell MJ, Shackelford P, Smith R, Schroeder K. Pharmacokinetics of clindamycin phosphate in the first year of life. J Pediatr. 1984;105:482-6.

Benakis A, Binh TQ, Keundjian A, Scheiwe MW. Pharmacokinetics/pharmacodynamics findings after repeated administration of artesunate thermostable suppositories (Rectocaps) in Vietnamese patients with uncomplicated malaria. Eur J Drug Metab Pharmacokinet. 2006;31:41-5.

Bennett JW, Pybus BS, Yadava A, Tosh D, Sousa JC, McCarthy WF, et al. Primaquine failure and cytochrome P-450 2D6 in Plasmodium vivax malaria. N Engl J Med. 2013;369:1381-2.

Bergqvist Y, Domeij-Nyberg B. Distribution of chloroquine and its metabolite desethylchloroquine in human blood cells and its implication for the quantitative determination of these compounds in serum and plasma. J Chromatogr. 1983;272(1):137-48.

Bergqvist Y, Eckerbom S. An improved gas chromatographic method for the simultaneous determination of chloroquine and two metabolites using capillary columns. J Chromatogr. 1984;306:147-53.

Bergqvist Y, Frisk-Holmberg M. Sensitive method for the determination of chloroquine and its metabolite desethylchloroquine in human plasma and urine by high-performance liquid chromatography. J Chromatogr. 1980;221:119-27.

Bergqvist Y, Hed C, Funding L, Suther A. Determination of chloroquine and its metabolites in urine: a field method based -on ion-pair extraction. Bull World Health Organ. 1985;63:893-8.

Bergqvist Y, Ericsson O, Rais M. Determination of chloroquine in dried blood spots on filter-paper. Importance of sample handling. Ther Drug Monit. 1986;8:211-31.

Bergqvist Y, Hellgren U, Churchill FC. High performance liquid chromatographic assay for the simultaneous monitoring of mefloquine and its acid metabolite in biological samples using protein precipitation and ion-pair extraction. J Chromatogr. 1988;432:253-63.

Bergqvist Y, Kabbani JA, Krysin B. High-Performance liquid chromatographic method for the determination of mefloquine and its carboxylic metabolite in $100 \mu \mathrm{l}$ capillary blood samples dried on paper. J Chemother. 1993;615:297-302.

Beringer PM, Owens H, Nguyen A, Benitez D, Rao A, D'Argenio DZ. Pharmacokinetics of doxycycline in adults with cystic fibrosis. Antimicrob Agents Chemother. 2012;56:70-4.

Berlin CM, Stackman BS, Vesell ES. Quinine-induced alterations in drug disposition. Clin Pharmacol Ther. 1975;18:670-9.

Bernakis A, Paris M, Plessas CH, Hein TT, Waller D White NJ. Pharmacokinetics of sodium artesunate after im or iv administration in severe malaria. Joint meeting of the American Society of Parasitologist and The American Society of Tropical Medicine and Hygiene, Atlanta, October 31-November 4, 1993.

Betuela I, Bassat Q, Kiniboro B, Robinson LJ, RosanasUrgell A, Stanisic D, et al. Tolerability and safety of primaquine in Papua New Guinean children 1 to 10 years of age. Antimicrob Agents Chemother. 2012;56:2146-9.

Beutler E. Drug induced haemolytic anaemia. Pharmacol Rev. 1969;21:73-103.

Beutler E, Dern RJ, Aliving AS. The hemolytic effect of primaquine IV. The relationship of the cell age to hemolysis. J Lab Clin Med. 1954;44:439-42.

Beutler E, Dern RJ, Aliving AS. The hemolytic effect of primaquine IV. An in vitro test for sensitivity of erythrocytes to primaquine. J Lab Clin Med. 1955;45:40.

Bhatia SC, Saraph YS, Revankar SN, Doshi KJ, Bharucha ED, Desai ND, Vaidya AB, Subrahmanyam D, Gupta $\mathrm{KC}$, Satoskar RS. Pharmacokinetics of primaquine in patients with $P$. vivax malaria. Eur J Clin Pharmacol. 1986;31:205-10.

Bindschedler M, Lefevre G, Ezzet F, Schaeffer N, Meyer I, Thomsen MS. Cardiac effects of co-artemether (artemether/lumefantrine) and mefloquine given alone or in combination to healthy volunteers. Eur J Clin Pharmacol. 2000;56:375-81.

Bindschedler M, Lefevre G, Degen P, Sioufi A. Comparison of the cardiac effect of the antimalarials 
co-artemether and halofantrine in healthy participants. Am J Trop Med Hyg. 2002;66:293-8.

Binh TQ, Ilett KF, Batty KT, Davis TM, Hung NC, Powell $\mathrm{SM}$, et al. Oral bioavailability of dihydroartemisinin in Vietnamse volunteers and in patients with falciparum malaria. Br J Clin Pharmacol. 2001;51:541-6.

Binh VQ, Chinh NT, Thanh NX, Cuong BT, Quang NN, Dai B, et al. Sex affects the steady-state pharmacokinetics of primaquine but not doxycycline in healthy subjects. Am J Trop Med Hyg. 2009;81:747-53.

Blockelman CR, Tan-ariya P. Efficacy of Fansider ${ }^{\mathrm{R}}$ against Plasmodium falciparum in continuous culture. Am J Trop Med Hyg. 1982;31(5):913-8.

Bocker R, Muhlberg W, Platt D, Estler CJ. Serum level, half-life and apparent volume of distribution of doxycycline in geriatric patients. Eur J Clin Pharmacol. 1986;30:105-8.

Boland ME, Roper SM, Henry JA. Complications of quinine poisoning. Lancet. 1985;1(8425):384-5.

Bolme P, Otto U. Dose dependence of the pharmacokinetics of quinidine. Eur J Clin Pharmacol. 1977;12:73-6.

Borrmann S, Sallas WM, Machevo S, Gonzalez R, Bjorkman A, Martensson A, et al. The effect of food consumption on lumefantrine bioavailability in African children receibing artemether-lumefantrine crushed or dispersible tablets (Coartem) for acute uncomplicated Plasmodium falciparum malaria. Tropical Med Int Health. 2010;15:434-41.

Botero D, Restrepo M, Montoya A. Prospective doubleblind trial of two different doses of mefloquine plus pyrimethaminesulfadoxine compared with pyrimethamine-sulfadoxine alone in the treatment of falciparum malaria. Bull World Health Organ. 1985;63:731-7.

Bouazza N, Pestre V, Jullien V, Curis E, Urien S, Salmon D, et al. Popution pharmacokinetics of clindamycin orally and intravenously administered in patients with osteomyelitis. Br J Clin Pharmacol. 2012;74:971-7.

Boudreau EF, Webster HK, Pavanand K, Thosingha L. Type II mefloquine resistance in Thailand. Lancet. 1982;2:1335.

Boudreau EF, Fleckenstein L, Pang MP, Childs GE, Schroeder AC, Ratnaratorn B, Pintuyothin P. Mefloquine kinetics in cured and recrudescent patients with acute falciparummalaria. Clin Pharmacol Ther. 1990;48(4):399-409.

Bourahla A, Martin C, Gimenez F, Singhasivanon V, Attanath P, Sabchearon A, et al. Stereoselective pharmacokinetics of mefloquine in young children. Eur J Clin Pharmacol. 1996;50:241-4.

Bradley D. Prophylaxis against malaria for travelers from the United Kingdom. BMJ. 1993;306:1247-52.

Brandling-Bennett AD, Oloo AJ, Khan B, Watkins WM. Failure of erythromycin to improve chloroquine treatment of Plasmodium falciparum malaria in Kenya. Trans R Soc Trop Med Hyg. 1988;82:363-5.

Brasseur P, Kouamouo J, Moyou-Somo R, Druilhe P. Multidrug resistant falciparum malaria in Cameroon in 1987-1988. I. Stable figures of prevalence of chloroquine-and quinine-resistant isolates in the original foci. Am J Trop Med Hyg. 1992;46(1):1-7.

Brasseur P, Vaillant MT, Olliaro PL. Anti-malarial drug safety information obtained through routine monitoring in a rural district of south-western Senegal. Malar J. 2012;11:402.

Bray PG, Mungthin M, Ridley RG, Ward SA. Access to hematin: the basis of chloroquine resistance. Mol Pharmacol. 1998;54:170-9.

Bregani ER, Tien TV, Figini G. Mefloquine-induced grand mal seizure in tubercular meningitis. Panminerva Med. 1998;40:345-6.

Briand V, Bottero J, Noel H, Masse V, Cordel H, Guerra J, et al. Intermittent treatment for the prevention of malaria during pregnancy in Benin: a randomized, open-label equivalence trial comparing sulfadoxinepyrimethamine with mefloquine. $J$ Infect Dis. 2009;200:991-1001.

Briolant S, Henry M, Oeuvray C, Amalvict R, Baret E, Didillon $\mathrm{E}$, et al. Absence of association between piperaquine in vitro responses and polymorphisms in the pfcrt, pfmdrl, pfmrp, and pfnhe genes in Plasmodium falciparum. Antimicrob Agents Chemother. 2010;54:3537-44.

Brockelman CR, Tan-ariya P, Bunnag D. Development of and in vitro microtest for the assessment of Plasmodium vivax sensitivity of chloroquine. Southeast Asian J Trop Med Public Health. 1989;20:41-7.

Brocks DR, Pasutto PM, Jamali F. Analytical and semipreparative high performance liquid chromatographic separation and assay of hydroxychloroquine enantiomers. J Chromatogr. 1992;581(1):83-92.

Brodie BB, Udenfriend S. The estimation of quinine in human plasma, with a note on the estimation of quinidine. J Pharmacol Exp Ther. 1943;74:154.

Brodie BB, Udenfriend S, Dill W, Chenkin T. The estimation of basic organic compounds in biological material III. Estimation by conversion to fluorescent compounds. J Biol Chem. 1947;168:319-25.

Brodie BB, Bear JE, Craig LC. Metabolic products of the cinchona alkaloids in human urine. $\mathrm{J}$ Biol Chem. 1951;188:567-81.

Brown HW, Neva FA. Malaria parasites of human beings. In: Basic clinical parasitology. 5th ed. Norwalk: Appleton-Century Crofts; 1983. p. 78-98.

Brown ND, Poon BT, Phillips LR. Identification and determination of a carboxylic acid metabolite of chloroquine in human urine by high performance liquid chromatography. J Chromatogr. 1989;487(1):189-96.

Brown-Awala EA, Thomas WO, Essien EE, Ette EI. Determination of saliva: total plasma chloroquine levels relationship by high performance liquid chromatography. Br J Clin Pharmacol. 1989;29(12):1135-9.

Bruce-Chwatt LJ. Classification of antimalarial drugs in relation to different stages in the life-cycle of the parasite: commentary on a diagram. Bull World Health Organ. 1962;27:287-90. 
Bruce-Chwatt LJ, Black RH, Cranfield CJ, Clyde DF, Peters W, Wernsdorfer WH. Chemotherapy of malaria. 2nd ed. Geneva: WHO; 1981.

Bryson HM, Goa KL. Halofantrine. Drugs. 1992;32(2):236-58.

Bunnag D, Harinasuta T. Quinine and quinidine in malaria in Thailand. Acta Leidensia. 1987a;55:163-6.

Bunnag D, Harinasuta T. The current status of drug resistance in malaria. In: Proceedings of 6th international congress of parasitology, Brisbane, 24-29 August $1987 b$.

Bunnag D, Harinasuta T, Pinichpongse S, Suntharasamai P. Effect of primaquine on gametocytes of Plasmodium falciparum in Thailand. Lancet. 1980;2:91.

Bunnag D, Harinasuta T, Vanijanonta S, Looareesuwan S, Chitamas S, Punnavut W, Jochim E. Slow-release quinidine in the treatment of chloroquine resistant falciparum malaria: a double-blind trial. Acta Leidensia. 1987a;55:129-38.

Bunnag D, Harinasuta T, Vanijanonta S, Looareesuwan S, Chittamas S, Punnavut W, Berth J, Druilhem P. Treatment of chloroquine-resistant falciparum malaria with a combination of quinine, quinidine and cinchonine (LA40221) in adults by oral and intravenous administration. Acta Leidensia. 1987b;55:139-49.

Bunnag D, Viravan C, Looareesuwan S. Double blind randomized clinical trial of two different regimens of oral artesunate in falciparum malaria. Southeast Asian J Trop Med Public Health. 1991a;22:534-8.

Bunnag D, Viravan C, Looareesuwan S, Karbwang J, Harinasuta T. Double blind randomized clinical trial of oral artesunate at once or twice daily dose in falciparum malaria. Southeast Asian J Trop Med Public Health. 1991b;22(4):539-43.

Bunnag D, Viravan C, Looareesuwan S, Looareesuwan S, Karbwang J, Harinnasuta T. Clinical trial of artesunate and artemether on multidrug resistant falciparum malaria in Thailand: a preliminary report. Southeast Asian J Trop Med Public Health. 1991c;22(3):380-5.

Bunnag D, Karbwang J, Harinasuta T. Artemether in the treatment of multiple drug resistant falciparum malaria. Southeast Asian J Trop Med Public Health. 1992a;23:762-7.

Bunnag D, Karwang J, Viravan C, Chittamas S, Harinasuta T. Clinical trials of mefloquine with tetracycline. Southeast Asian J Trop Med Public Health. 1992b;3(3):377-82.

Bunnag D, Karbwang J, Chittamas S, Harinasuta $\mathrm{T}$. Intramuscular artemether in female patients with uncomplicated falciparum malaria. Southeast Asian J Trop Med Public Health. 1993a;24:49-52.

Bunnag D, Viravan C, Karwang J, Looareesuwan S, Chittamas S, Harinasuta T, Horton J, Serville P. Clinical trials with halofantrine in acute uncomplicated falciparum malaria in Thailand. Southeast Asian J Trop Med Public Health. 1993b;24(1):43-8.

Bunnag D, Karbwang J, Thanavibul A, Chittamas S, Ratanapongse Y, Chalermrut K, Na-Bangchang K,
Harinasuta T. High dose primaquine in primaquine resistant vivax malaria. Trans $\mathrm{R}$ Soc Trop Med Hyg. 1994;88(2):218-9.

Bustos MD, Gay F, Diquet B, Tomare P, Warot D. The pharmacokinetics and electrocardiographic effects of chloroquine in healthy subjects. Trop Med Parasitol. 1994;45:83-6.

Bustos DG, Lazaro JE, Gay F, Pottier A, Laracas CJ, Traore B, et al. Pharmacokinetics of sequential and simultaneous treatment with the combination chloroquine and sulfadoxine-pyrimethamine in acute uncomplicated Plasmodium falciparum malaria in the Philippines. Tropical Med Int Health. 2002;7:584-91.

Butler AR. Artemisnin (Qinghaosu): a new type of antimalarial drug. Chem Soc Rew. 1992;21:85-90.

Byakika-Kibwika P, Lamorde M, Mayanja-Kizza H, Merry C, Colebunders B, Van Geertruyden JP. Update on the efficacy, effectiveness and safety of artemetherlumefantrine combination therapy for treatment of uncomplicated malaria. Ther Clin Risk Manage. 2010;6:11-20.

Byakika-Kibwika P, Lamorde M, Mayito J, Nabukeera L, Mayanja-Kizza H, Katabira E, et al. Pharmacokinetics and pharmacodynamics of intravenous artesunate during severe malaria treatment in Ugandan adults. Malar J. 2012a;11:132.

Byakika-Kibwika P, Lamorde M, Mayito J, Nabukeera L, Namakula R, Mayanja-Kizza H, et al. Significant pharmacokinetic interactions between artemether/ lumefantrine and efavirenz or nevirapine in HIVinfected Ugandan adults. J Antimicrob Chemother. 2012b;67:2213-21.

Byakika-Kibwika P, Lamorde M, Okaba-Kayom V, Mayanja-Kizza H, Katabira E, Hanpithakpong W, et al. Lopinavir/ritonavir significantly influences pharmacokinetic exposure of artemether/lumefantrine in HIV-infected Ugandan adults. J Antimicrob Chemother. 2012c;67:1217-23.

Carson PE. Enzymatic deficiency in primaquine-sensitive erythrocytes. Science. 1956;124:484.

Carson PE, Hohl R, Nora MV. Toxicology of the 8-aminoquinolines and genetic factors associated with their toxicity in man. Bull World Health Organ. 1981;59:427-43.

Chantavanich P, Looareesuwan S, White NJ, Warrell DA, Warrell MJ, Giovanti JH, Von Brewdow J. Intragastric mefloquine is absorbed rapidly in patients with cerebral malaria. Am J Trop Med Hyg. 1985;34:1028-36.

Chanthap L, Tsuyuoka R, Na-Bangchang K, Nivanna N, Suksom D, Sovannarith T, et al. Investigation of bioavailability, pharmacokinetics and safety of new pediatric formulations of artesunate and mefloquine. Southeast Asian J Trop Med Public Health. 2005;36:34-43.

Charles BG, Blomgren A, Nasveld PE, Kitchener SJ, Jensen A, Gregory RM, et al. Population pharmacokinetics of mefloquine in military personnel for prophylaxis against malaria infection during field deployment. Eur J Clin Pharmacol. 2007;63:271-8. 
Charoenlarp P, Areekul S, Harinasuta T, Sirivovasarn P. The haemolytic effect of a single dose of $45 \mathrm{mg}$ of primaquine in G6PD deficient Thais. J Med Assoc Thail. 1972;55:632.

Charoenlarp P, Areekul S, Pholpothi T, Harinasuta T. The cause of primaquine induced hemolysis in G6PD deficient Thais. J Med Assoc Thail. 1973;56:392.

Chaulet JF, Robet Y, Prevosto JM, Soares O. Simultaneous determination of chloroquine and quinine in human biological fluids by high-performance liquid chromatography. J Chromatogr. 1993;613:330-10.

Chawira AN, Warhurst DC. The effect of artemisinin combined with standard antimalarials against chloroquineresistant strains of Plasmodium falciparum in vitro. J Trop Med Hyg. 1987a;90:1-8.

Chawira AN, Warhurst DC. The effects of artemisinin combined with standard chloroquine-resistant strains of Plasmodium falciparum in vitro. J Trop Med Hyg. 1987b;90:1-8.

Chawira AN, Warhurst DC, Peters W. Artemisinin (qinghaosu) combinations against sensitive and chloroquine-resistant Plasmodium falciparum in vitro. Trans R Soc Trop Med Hyg. 1986a;80:335.

Chawira AN, Warhurst DC, Peters W. Drug combination studies with qinghaosu (artemisinin) against sensitive and resistant strains of rodent malaria. Trans $\mathrm{R}$ Soc Trop Med Hyg. 1986b;80:334-5.

Chawira AN, Warhurst DC, Robinson BL, Peters W. The effect of combinations of qinghaosu (artemisinin) with standard antimalarial drugs in the suppressive treatment of malaria in mice. Trans R Soc Trop Med Hyg. 1987;81:554-8.

Checchi F, Piola P, Fogg C, Bajunirwe F, Biraro S, Grandesso F, et al. Supervised versus unsupervised antimalarial treatment with six-dose artemetherlumefantrine: pharmacokinetic and dosage-related findings from a clinical trial in Uganda. Malar J. 2006;5:59.

Chester AC, Sandroni P. Case report: peripheral polyneuropathy and mefloquine prophylaxis. Am J Trop Med Hyg. 2011;85:1008-9.

Chevli R, Fitch CD. The antimalarial drug mefloquine binds to membrane phospholipids. Antimicrob Agents Chemother. 1982;21:581-6.

China Cooperative Research Group on Qinghaosu and its Derivatives as Antimalarials. Clinical studies on the treatment of malaria with qinghaosu and its derivatives. J Tradit Chin Med. 1982a;2:17-24.

China Cooperative Research Group on Qinghaosu and its Derivatives as Antimalarials. Clinical studies on the treatment of malaria with qinghaosu and its derivatives. J Tradit Chin Med. 1982b;2:25-30.

China Cooperative Research Group on Qinghaosu and its Derivatives as Antimalarials. Clinical studies on the treatment of malaria with qinghaosu and its derivatives. J Tradit Chin Med. 1982c;2:31-44.

China Cooperative Research Group on Qinghaosu and its Derivatives as Antimalarials. Clnical studies on the treatment of malaria with qinghaosu and its derivatives. J Tradit Chin Med. 1982d;2:45-50.

China Cooperative Research Group on Qinghaosu and its Derivatives as Antimalarials. Metabolism and pharmacokinetics of qinghaosu and its derivatives. J Tradit Chin Med. 1982e;2(1):25-30.

Chongsuphajaisiddhi T, Sabcharoen A, Attanath P. In vivo and in vitro sensitivity of falciparum malaria to quinine in Thai children. Ann Trop Paediatr. 1981;1:21-6.

Chongsuphajaisiddhi T, Sabcharoen A, Attanath P. Treatment of quinine resistant falciparum malaria in Thai children. Southeast Asian J Trop Med Public Health. 1983;14(3):357-62.

Chongsuphajaisiddhi T, Sabcharoen P, Chantavanich P, Singhasivanon V, Attanath P, Wernsdorfer WH, Sheth UK. A phase-III clinical trial of mefloquine in children with chloroquine-resistant falciparum malaria in Thailand. Bull World Health Organ. 1987;65(2):223-6.

Churchill FC, Mount DL, Schwartz IK. Determination of chloroquine and its major metabolite in blood using perfluoroacylation by fused-silica capillary gaschromatography with nitrogen-sensitive detection. J Chromatogr. 1983;274:11-120.

Cinchona bark http://historyofmalaria.com/2010/02/ cinchona-bark/

Claessen FA, van Boxtel CJ, Perenboom RM, Tange RA, Wetsteijn JC, Kager PA. Quinine pharmacokinetics: ototoxic and cardiotoxic effects in healthy Caucasian subjects and in patients with falciparum malaria. Tropical Med Int Health. 1998;3:482-9.

Clark AM, Baker JK, McChesney JD. Excretion, distribution, and metabolism of primaquine in rats. $\mathrm{J}$ Pharm Sci. 1984;73:502-6.

Clyde DF. Clinical problems associated with the use of primaquine as a tissue schizontocidal and gametocytocidal drugs. Bull World Health Organ. 1981;59:391-3.

Clyde DF, McCarthy VC. Radical cure of chesson strain vivax malaria in man by 7 , not 14 , days of treatment with primaquine. Am J Trop Med Hyg. 1977;26(3):562-3.

Collignon $\mathrm{P}$. Chloroquine resistance in Plasmodium vivax. J Infect Dis. 1991;164:222-3.

Consensus conference. Newborn screening for sickle cell disease and other hemoglobinopathies. JAMA. 1987;258:1205-9.

Contacos PG, Coatney GR, Colins WE, Briesch PE, Peter $\mathrm{MH}$. Five day primaquine therapy-An evaluation of radical curative activity against vivax malaria infection. Am J Trop Med Hyg. 1973;22(6):693-5.

Couet W, Laroche R, Floch JJ, Istin B, Fourtillan JB, Sauniere JF. Pharmacokinetics of quinine and doxycycline in patients with acute falciparum malaria: a study in Africa. Ther Drug Monit. 1991;13:496-501.

Crevoisier CA, Joseph I, Fischer M, Graf H. Influence of hemodialysis on plasma concentration-time profiles of mefloquine in two patients with endstage renal disease: a prophylactic drug monitoring study. Antimicrob Agents Chemother. 1995;39:1892-5. 
Crouthamel WG, Kowarski B, Narang PK. Specific serum quinidine assay by high-perfomance liquid chromatography. Clin Chem. 1977;23(11):2030-3.

Cunha BA, Sibley CM, Ristuccia AM. Doxycycline. Ther Drug Monit. 1982;4:115-35.

Cuong BT, Binh VQ, Dai B, Duy DN, Lovell CM, Rieckmann $\mathrm{KH}$, et al. Does gender, food or grapefruit juice alter the pharmacokinetics of primaquine in healthy subjects? Br J Clin Pharmacol. 2006;61:682-9.

Da Silva Morais M, Augusto O. Peroxidation of the antimalarial drug primaquine: characterization of a benzidine-like metabolite with methaemoglobinforming activity. Xenobiotica. 1993;23(2):133-9.

Dahl EL, Shock JL, Shenai BR, Gut J, DeRisi JL, Rosenthal PJ. Tetracyclines specifically target the apicoplast of the malaria parasite Plasmodium falciparum. Antimicrob Agents Chemother. 2006;50:3124-31.

Dassonville-Klimpt A, Jonet A, Pillon M, Mullié C, Sonnet P. Mefloquine derivatives: synthesis, mechanisms of action, antimicrobial activities. In: Mendez-Vilas A, editor. Science against microbial pathogens: communicating current research and technological advances. Badajoz: Formatex Research Center; 2011. p. 23-35.

Data JL, Wilkinson GR, Nies AS. Interaction of quinidine with anticonvulsant drugs. New Engl J Med. 1976;294(13):699-702.

Davis TME, White NJ, Looareesuwan S, Silamut K, Warrell DA. Quinine pharmacokinetics in cerebral malaria: predicted plasma concentrations after rapid intravenous loading using a two-compartment model. Trans R Soc Trop Med Hyg. 1988;82:542-7.

Davis TM, Phuong HL, Ilett KF, Hung NC, Batty KT, Phuong VD, et al. Pharmacokinetics and pharmacodynamics of intravenous artesunate in severe falciparum malaria. Antimicrob Agents Chemother. 2001;45:181-6.

Davis TM, England M, Dunlop AM, Page-Sharp M, Cambon N, Keller TG, et al. Assessment of the effect of mefloquine on artesunate pharmacokinetics in healthy male volunteers. Antimicrob Agents Chemother. 2007;51:1099-101.

De Souza JM. A phase I clinical trial of mefloquine in Brazilian male subjects. Bull World Health Organ. 1983;61:809-14.

De Souza JM. Mefloquine clinical trials therapeutical experience with mefloquine alone and combination (MSP) in Brazilian male subjects with falciparum malaria. Mem do Inst Oswaldo. 1986;81(Suppl 2):259-68.

De Souza JM, Sheth UK, De Oliveira RMG, Roulet H, De Souza SD. An open randomized, phase III clinical trial of mefloquine and of quinine plus sulfadoxinepyrimethamine in the treatment of symptomatic falciparum malaria in Brazil. Bull World Health Organ. 1985;63:603-9.

De Souza JM, Heizmann P, Schwatrz DE. Single-dose kinetics of mefloquine in Brazilian males subjects. Bull World Health Organ. 1987;65(3):353-6. del Carmen C-PM, Lujan M, Flores-Murrieta FJ. Evaluation of gender in the oral pharmacokinetics of clindamycin in humans. Biopharm Drug Dispos. 2008;29:427-30.

Deloron P, Lepers JP, Andriamangatiana-Rason D, Coulangers P. Short-term oral cinchona alkaloids regimens for treatment of falciparum malaria in Madagascar. Trans R Soc Trop Med Hyg. 1990;84:54.

Denis MB, Davis TM, Hewitt S, Incardona S, Nimol K, Fandeur T, et al. Efficacy and safety of dihydroartemisinin-piperaquine (Artekin) in Cambodian children and adults with uncomplicated falciparum malaria. Clin Infect Dis. 2002;35:1469-76.

Dern RJ, Beutler E, Aliving AS. The hemolytic effect of primaquine II. The natural cause of the hemolytic anemia and the mechanisms of its self-limited character. J Lab Clin Med. 1954;44:171.

Dern RJ, Beutler E, Alving AS. The hemolytic effect of Primaquine. V. Primaquine sensitivity as a manifestation of a multiple drug sensitivity. J Lab Clin Med. 1955;45:40.

Desjardins RE, Pamplin CL, von Bredow J, Barry KG, Canfield CJ. Kinetics of a new antimalarial, mefloquine. Clin Pharmacol Ther. 1979;26:372-9.

Deye GA, Miller RS, Miller L, Salas CJ, Tosh D, Macareo L, et al. Prolonged protection provided by a single dose of atovaquone-proguanil for the chemoprophylaxis of Plasmodium falciparum malaria in a human challenge model. Clin Infect Dis. 2012;54:232-9.

Dicko A, Diallo A, Tembine I, Dicko Y, Dara N, Sidibe Y, et al. Intermittent preventive treatment of malaria provides substantial protection against malaria in children already protected by an insecticide-treated bednet in Mali: a randomized, double-blind, placebo-controlled trial. PLoS Med. 2011;8:e1000407.

Dixon KE, Willaims RG, Pongsupat T, Pitaktong U, Phintuyothin P. A comparative trial of mefloquine and Fansidar $^{\mathrm{R}}$ in the treatment of falciparum malaria: failure of Fansidar ${ }^{\mathrm{R}}$. Trans $\mathrm{R}$ Soc Trop Med Hyg. 1982;76(5):664.

Dixon KE, Pitaktong U, Bamnetpandh S, Teopipithaporn S, Na-Nakorn A. Treatment of an acute case of Plasmodium malariae malaria with mefloquine. Am J Trop Med Hyg. 1983;32(3):631-2.

Dixon KE, Pitaktong U, Phintuyothin P. A clinical trial of mefloquine in the treatment of Plasmodium vivax malaria. Am J Trop Med Hyg. 1985;34(3):435-7.

Djimde AA, Tekete M, Abdulla S, Lyimo J, Bassat Q, Mandomando I, et al. Pharmacokinetic and pharmacodynamic characteristics of a new pediatric formulation of artemether-lumefantrine in African children with uncomplicated Plasmodium falciparum malaria. Antimicrob Agents Chemother. 2001;55:3994-9.

Doberstyn EB, Phintuyothin P, Noeypatimanondh S, Teerakiatkamjorn C. Single-dose therapy of falciparum malaria with mefloquine or pyrimethaminesulfadoxine. Bull World Health Organ. 1979;57:275-9.

Dondorp A, Nosten F, Stepniewska K, Day N, White N. Artesunate versus quinine for treatment of severe 
falciparum malaria: a randomized trial. Lancet. 2005;366:717-25.

Dondorp AM, Fanello CI, Hendriksen IC, Gomes E, Seni A, Chhaganlal KD, et al. Artesunate versus quinine in the treatment of severe falciparum malaria in African children (AQUAMAT): an open-label, randomized trial. Lancet. 2010;376:1647-57.

Dourado H, Albuquerque BC, Alecrim W, Alecrim MG, Ferreira I. Comparison of Ro 21-5998 (mefloquine) and Fansidar $^{\mathrm{R}}$ (pyrimethamine plus sulfadoxine) for the treatment of Plasmodium falciparum malaria in the Amazon. In: Periti P, Gialdroni-Grassi D, editors. Current chemotherapy and immunotherapy. 2 Washington, DC: American Society for Microbiology; 1982. p. $1042-4$.

Drayer DE, Restivo K, Reidenberg MM. Spcific determination of quinidine and (3S)-3-hydroxyquine in human serum by high-pressure liquid chromatography. J Lab Clin Med. 1977;90:816-22.

Druilhe P, Brandicourt O, Chongsuphajaisiddhi T, Berthe J. Activity of a combination of three cinchona bark alkaloids against Plasmodium falciparum in vitro. Antimicrob Agents Chemother. 1988;32:250-4.

Dua VK, Sarin R. Determination of quinine in serum, plasma, red blood cells and whole blood in healthy and Plasmodium falciparum malaria cases by highperformance liquid chromatograpny. J Chromatogr. 1993;614:87-93.

Ducharme J, Farinotti R. Clinical pharmacokinetics and metabolism of chloroquine. Focus on recent advancements. Clin Pharmacokinet. 1996;31:257-74.

Dutta GP, Bajpai R, Vishwakarma RA. Comparison of antimalarial efficacy of artemisinin (qinghaosu) and arteether against Plasmodium cynomolgi B infection in monkeys. Trans $\mathrm{R}$ Soc Trop Med Hyg. 1989;83:56-7.

Duy N, Hoan DB, Dung NP, Huong NV, Binh LN, Son MV, Meshnick SR. Treatment of malaria in Vietnam with oral artemisinin. Am J Trop Med Hyg. 1993;48(3):398-402.

Dyer JR, Davis TM, Giele C, Annus T, Garcia-Webb P, Robson J. The pharmacokinetics and pharmacodynamics of quinine in the diabetic and nondiabetic elderly. $\mathrm{Br}$ J Clin Pharmacol. 1994;38:205-12.

Earle DP, Bigelow FS, Subrod CG, Rane CA. Studies on the chemotherapy of the human malarias: IX. Effect of primaquine on the blood cells of man. J Clin Invest. 1948;27:121.

Ebringer A, Heathcote G, Baker J, Waller M, Shanks GD, Edstein MD. Evaluation of the safety and tolerability of a short higher-dose primaquine regimen for presumptive anti-relapse therapy in healthy subjects. Trans R Soc Trop Med Hyg. 2011;105:568-73.

Edgcomb JH, Arnold J, Yount Jr EH, Alving AS, Eichelberger L. Primaquine, SN 13272, a new curative agent in vivax malaria: a preliminary report. J Natl Mal Soc. 1950;9:285-92.

Edlund PO, Westerlund D, Carlqvist J, Wu BL, Jin JH. Determination of artesunate and dihydroartemisinin in plasma by liquid chromatography with post-column derivatization and UV detection. Acta Pharm Sci. 1984;21:223-34.

Edstein M, Stace J, hann F. Quantification of quinine in human serum by high-performance liquid chromatography. J Chromatogr. 1983;278:445-51.

Edstein MD, Veenedaal JR, Newman K, Hyslop $\mathrm{R}$. Excretion of chloroquine, dapsone and pyrimethamine in human milk. $\mathrm{Br} \mathrm{J}$ Clin Pharmacol. 1986;22(6):733-5.

Edstein MD, Veenendaal JR, Hyslop R. Excretion of mefloquine in human breast milk. Chemother. 1988;34:165-9.

Edwards GE, Davies AJ, Phillips RE, Looareesuwan S, Karbwang J, White NJ, Warrell DA. Plasma concentrations and toxicity of chloroquine after slow intravenous in fusion in patients with falciparum malaria. Ann Trop Med Parasitol. 1987;81(2):79-84.

Edwards GE, Looareesuwan S, Davies AJ, Wattanagoon Y, Phillips RE, Warrell DA. Pharmacokinetics of chloroquine in Thais: plasma and red-cell concentration following an intravenous infusion to healthy subjects and patients with Plasmodium vivax malaria. Br J Clin Pharmacol. 1988;25:477-85.

Edwards G, McGrath CS, Ward SA, Supanaranond W, Pukrittayakamee S, Davis TME, White NJ. Interaction among primaquine, malaria infection and other antimalarials in Thai subjects. Br J Clin Pharmacol. 1993;35:193-8.

Eizaki K, Ahmed MEIM, Eihassan AM, Yousif MA, Fahor AH. Acute gluteal bascess following intramuscular chloroquine injection: a clinical and experimental study. J Trop Med Hyg. 1991;94:206-9.

Ekue JMK, Ulrich AM, Rwawogo-Atenyi J, Sheth UK. A double blind comparative clinical trial of mefloquine and chloroquine in symptomatic falciparum malaria. Bull World Health Organ. 1983;61:713-8.

Ekue JMK, Simooya OO, Sheth UK, Wernsdorfer WH, Njelesani EK. A double-blind clinical trial of a combination of mefloquine, sulfadoxine and pyrimethamine in symptomatic falciparum malaria. Bull World Health Organ. 1985;63:339-43.

Ekue JMK, Phiri DED, Sheth UK, Mukunyandela M. A double-blind trial of a fixed combination of mefloquine plus sulfadoxine-pyrimethamine compared with sulfadoxine alone in symptomatic falciparum malaria. Bull World Health Organ. 1987;65(3):369-73.

Elmes NJ, Bennett SM, Abdalla H, Carthew TL, Edstein MD. Lack of sex effect on the pharmacokinetics of primaquine. Am J Trop Med Hyg. 2006;74:951-2.

Endoh YS, Yoshimura H, Sasaki N, Ishihara Y, Sasaki H, Nakamura S, Inoue Y, Nishikawa M. High performance liquid chromatographic determination of pamaquine, primaquine and carboxyprimaquine in calf plasma using electrochemical detection. J Chromatogr. 1992;579:123-9.

Ericsson CD, Feldman S, Pickering LK, Cleary TG. Influence of subsalicylate bismuth on absorption of doxycycline. JAMA. 1982;247:2266-7. 
Escande C, Chevalier P, Verdier F, Bourdon R. Sensitive radioimmunoassay and enzyme-linked immunosorbent assay for the simultaneous determination of chloroquine and its metabolites in biological fluids. J Pharm Sci. 1990;9(1):23-7.

Esseim EE, Ifudu ND. Residual chloroquine and metabolites in man as a sequel of previous chloroquine medications: a urinary secretion study and its significance. J Trop Med Hyg. 1984;87(3):131-6.

Essien EE, Ette EI, Thomas WO, Brown-Awala EA. Chloroquine disposition in hypersensitive and non-hypersensitive subjects and its significance in chloroquine- induced pruritus. Eur J Drug Metab Pharmacokinet. 1989;14(1):71-7.

Ette EI, Essien EE, Brown-Awala EE. Pharmacokinetics of chloroquine: saliva and plasma levels relationship. Eur J Drug Metab Pharmacokinet. 1986a;11:275-81.

Ette EI, Essein EE, Brown-Awala EE. Pharmacokinetics of chloroquine: saliva and plasma levels relationship. Eur J Drug Metab Pharmacokinet. 1986b;11(4):275-81.

Ette EI, Brown-Awala EA, Essien EE. Chloroquine elimination in humans: effect of low-dose cimetidine. J Clin Pharmacol. 1987a;27:813-6.

Ette EI, Brown-Awala A, Essein EE. Effect of ranitidine on chloroquine disposition. Drug Inter Clin Pharm. 1987b;21(9):732-4.

Ette EI, Ogonor JI, Essien EE. Passage of chloroquine into semen. Br J Clin Pharmacol. 1988;26:179-82.

Ette EI, Essien EE, Thomas WOA, Brown-Awala EA. Pharmacokinetics of chloroquine and some of its metabolites in healthy volunteers: a single dose study. J Clin Pharmacol. 1989;29:457-62.

European Medicines Agency. Eurartesim (dihydroartemisinin/ piperaquine) $20 \mathrm{mg} / 160 \mathrm{mg}$ and $40 \mathrm{mg} / 320 \mathrm{mg}$ filmcoated tablets: EU summary of product characteristics. London. http://www.ema.europa.eu/docs/en_GB/docu ment_library/EPAR___Product_Information/human/ 001199/WC500118113.pdf

Ezzet F, Mull R, Karbwang J. Population pharmacokinetics and therapeutic response of CGP 56697 (artemether+benflumetol) in malaria patients. $\mathrm{Br}$ J Clin Pharmacol. 1998;46:553-61.

Ezzet F, van Vugt M, Nosten F, Looareesuwan S, White NJ. Pharmacokinetics and pharmacodynamics of lumefantrine (benflumetol) in acute falciparum malaria. Antimicrob Agents Chemother. 2000;44:697-704.

Fattinger K, Vozeh S, Ha R, Borner M, Follath F. Population pharmacokinetics of quinidine. $\mathrm{Br}$ J Clin Pharmacol. 1991;31:279-86.

Fehintola FA, Scarsi KK, Ma Q, Parikh S, Morse GD, Taiwo B, et al. Nevirapine-based antiretroviral therapy impacts artesunate and dihydroartemisinin disposition in HIV-infected Nigerian adults. AIDS Res Treat. 2012;2012:703604.

Fiaccadori E, Maggiore U, Rotelli C, Giacosa R, Parenti E, Cabassi A, et al. Thrombotic-thrombocytopenic purpura following malaria prophylaxis with mefloquine. J Antimicrob Chemother. 2006;57:160-1.
Finielz P, Gendoo Z, Chuet C, Guiserix J. Interaction between cyclosporin and chloroquine. Nephron. 1993;65:333.

Flaherty Jr JF, Gatti G, White J, Bubp J, Borin M, Gambertoglio JG. Protein binding of clindamycin in sera of patients with AIDS. Antimicrob Agents Chemother. 1996;40:1134-8.

Flaherty JF, Rodondi LC, Guglielmo BJ, Fleishaker JC, Townsend RJ, Gambertoglio JG. Comparative pharmacokinetics and serum inhibitory activity of clindamycin in different dosing regimens. Antimicrob Agents Chemother. 1988;32:1825-9.

Fletcher A, Di Shepherd R. Use of (+)-mefloquine for the treatment of malaria. Patent no. WO 1998039003 AI, 6 Mar 1998.

Fletcher KA, Canning MV, Gilles HM. Metabolites of primaquine and their effects on erythrocytes. Trans R Soc Trop Med Hyg. 1977;71:111-2.

Fletcher KA, Evans DAP, Gilles HM, Greaves J, Bunnag D, Harinasute T. Studies on the pharmacokinetics of primaquine. Bull World Health Organ. 1981a;59(3):407-12.

Fletcher KA, Price DA, Gilles HM, Greaves J, Bunnag D, Harinasuta T. Studies on the pharmacokinetics of primaquine. Bull World Health Organ. 1981b;59:407-12.

Fletcher KA, Barton PE, Kelly JA. Studies on the mechanisms of oxidation in the erythrocyte by metabolites of primaquine. Biochem Pharmacol. 1988;37(13):2683-90.

Franke U, Proksch P, Muller M, Risher T, Ehninger G. Drug monitoring of quinine by HPLC in cerebral malaria with acute renal failure treated by haemofiltration. Eur J Clin Pharmacol. 1987;33:293-6.

Franssen G, Rouviex B, Lebras J, Bauchet J, Verdier F, Michon C, Bricarire F. Divided-dose kinetics of mefloquine in man. Br J Clin Pharmacol. 1989;28:179-84.

Fraser IM, Strother A, Tilton BF. Methaemoglobin formation in normal and G6PD deficient human erythrocytes by primaquine and mouse liver microsomes system. Fed Proc. 1976;35:244.

Frisk-Holmberg M, Bergqvist Y, Domeij-Nyberg B, Hellstrom L, Jansson F. Chloroquine serum concentrations and side effects: evidence of dose-dependent kinetics. Clin Pharmacol Ther. 1979;25:345-50.

Frisk-Holmberg M, Bergqvist Y, Englund ULF. Chloroquine intoxication. $\mathrm{Br} \mathrm{J}$ Clin Pharmacol. 1983;15:502-3.

Frisk-Holmberg M, Bergqvist Y, Englund U. Chloroquine in toxication. Br J Clin Pharmacol. 1984;15:502-3.

Fuller SJ, Naraqi S, Gilessi G. Paranoid psychosis related to mefloquine antimalarial prophylaxis. Papua New Guinea. Med J. 2002;45:219-21.

Furner RL, Brown GB, Scott JW. A method for differentiation and analysis of quinine and quinidine by gas chromatography/mass spectrometry. J Anal Toxical. 1981;5:275-8.

Ganesan S, Tekwani BL, Sahu R, Tripathi LM, Walker LA. Cytochrome P(450)- dependent toxic effects of 
primaquine on human erythrocytes. Toxicol Appl Pharmacol. 2009;241:14-22.

Garavelli PL, Corti E. Chloroquine resistance in Plasmodium vivax: the first case in Brazil. Trop Med Hyg. 1992;89:128.

Gargano N, Ubben D, Tommasini S, Bacchieri A, Corsi M, Bhattacharyya PC, et al. Therapeutic efficacy and safety of dihydroartemisinin-piperaquine versus artesunate-mefloquine in uncomplicated Plasmodium falciparum malaria in India. Malar J. 2012;11:233.

Garin D, Lamarque D, Ringwald P, Dupuy O, Chaulet JF, Chapalainm C, Flechaere A. Efficacy of chloroquineproguanil chemoprophylaxis against malaria in the Central African Republic. Trans R Soc Trop Med Hyg. 1993;87:304-5.

Garraffo A, Dellamonica P, Fournier JP, Lapalus P, Bernard E. The effect of rifampicin on the pharmacokinetics of doxycycline. Infection. 1988;16:297-8.

Gasasira AF, Kamya MR, Achan J, Mebrahtu T, Kalyango JN, Ruel T, et al. High risk of neutropenia in HIVinfected children following treatment with artesunate plus amodiaquine for uncomplicated malaria in Uganda. Clin Infect Dis. 2008;46:985-91.

Gatti G, Flaherty J, Bubp J, White J, Borin M, Gambertoglio J. Comparative study of bioavailabilities and pharmacokinetics of clindamycin in healthy volunteers and patients with AIDS. Antimicrob Agents Chemother. 1993;37:1137-43.

Geary TG, Bonanni LC, Jensen JB. Effects of combinations of quinoline-containing antimalarials on Plasmodium falciparum in culture. Ann Trop Med Parasitol. 1986;3:285-91.

George JN, Sears DA, McCurdy P, Conrod ME. Primaquine sensitive in Caucasians: hemolytic reactions induced by primaquine in G6PD deficient subjects. J Lab Clin Med. 1967;70:80.

George W, Gary WL, Laurena PP, Alban P, Sangalang R, Panoa CP. Chloroquine and quinine: a randomized, double-blind comparison of efficacy and side effects in the treatment of Plasmodium falciparum malaria in the Philippines. Trans R Soc Trop Med Hyg. 1988;82:205-8.

German PI, Aweeka FT. Clinical pharmacology of artemisinin-based combination therapies. Clin Pharmacokinet. 2008;47:91-102.

German P, Greenhouse B, Coates C, Dorsey G, Rosenthal PJ, Charlebois E, et al. Hepatotoxicity due to a drug interaction between amodiaquine plus artesunate and efavirenz. Clin Infect Dis. 2007;44:889-91.

German P, Parikh S, Lawrence J, Dorsey G, Rosenthal PJ, Havlir D, et al. Lopinavir/ritonavir affects pharmacokinetic exposure of artemether/lumefantrine in HIVuninfected healthy volunteers. J Acquir Immune Defic Syndr. 2009;51:424-9.

Giboda M, Vanista J, Dastych P. The first report of Plasmodium falciparum resistant to chloroquine plus sulphadoxine/pyrimethamine in Rwanda. Trans R Soc Trop Med Hyg. 1988;82:383.
Gilder ME, Hanpithakphong W, Hoglund RM, Tarning J, Htun Win H, Hilda N, Chu CS, Bancone G, Carrara VI, Singhasivanon P, White NJ, Nosten F, McGready R. Primaquine pharmacokinetics in lactating women and breastfed infant exposures. Clin Infect Dis. 2018;24. https://doi.org/10.1093/cid/ciy235. [Epub ahead of print].

Gimenez F, Farinotii R, Thuilier A, Hazebrouch G, Wainer IW. Determination of the enantiomers of mefloquine in plasma and whole blood using a coupled achiral-chiral high performance liquid chromatographic system. J Chromatogr. 1990;529:339-46.

Gimenez F, Pennie RA, Koren G, Crevoisier C, Wainer IW, Farinotti R. Stereoselective pharmacokinetics of mefloquine in healthy Caucasians after multiple doses. J Pharm Sci. 1994;83:824-7.

Gimnig JE, MacArthur JR, M'Bang'ombe M, Kramer MH, Chizani N, Stern RS, et al. Severe cutaneous reactions to sulfadoxine-pyrimethamine and trimethoprim-sulfamethoxazole in Blantyre District, Malawi. Am J Trop Med Hyg. 2006;74:738-43.

Goldenberg AM, Wexler LF. Quinine overdose: review of toxicity and treatment. Clin Cardiol. 1988;11:716-8.

Golenser J, Waknine JH, Krugliak M, Hunt NH, Grau GE. Current perspectives on the mechanism of action of artemisinins. Int J Parasitol. 2006;36:1427-41.

Gosling RD, Gesase S, Mosha JF, Carneiro I, Hashim R, Lemnge $\mathrm{M}$, et al. Protective efficacy and safety of three antimalarial regimens for intermittent preventive treatment for malaria in infants: a randomised, doubleblind, placebo-controlled trial. Lancet. 2009;374:1521-32.

Gotsman I, Azaz-Livshits T, Fridlender Z, Muszkat M, Ben-Chetrit E. Mefloquine-induced acute hepatitis. Pharmacotherapy. 2000;20:1517-9.

Grahnen A, Olsson B, Johansson G, Eckernas SA. Doxycycline carrageenate - an improved formulation providing more reliable absorption and plasma concentrations at high gastric $\mathrm{pH}$ than doxycycline monohydrate. Eur J Clin Pharmacol. 1994;46:143-6.

Greaves J, Evans DAP, Gilles HM, Baty JD. A selected ion monitoring assay for primaquine in plasma and urine. Biomed Mass Spectrom. 1979;6:102-12.

Greaves J, Evans DAP, Gilles HM, Fletcher KA, Bunnag D, Harinasuta T. Plasma kinetics and urinary excretion of primaquine in man. Br J Clin Pharmacol. 1980;10:399-405.

Greenblatt DJ, Pfeifer HJ, Ochs HR, Franke K, MacLaughlin DS, Smith TW, Koch-wesser J. Pharmacokinetics of quinidine in humans after intravenous, intramuscular and oral administration. J Pharmacol Exp Ther. 1977;202(2):365-78.

Grendel D, Verdier F, Richard-Lenoble D, Nardou M. Interaction of cholestyramine and chloroquine. Arch Fr Pediatr. 1990;47(5):387-8.

Grieco A, Vecchio FM, Natale L, Gasbarrini G. Acute fatty liver after malaria prophylaxis with mefloquine. Lancet. 1999;353:295-6. 
Grindel JM, Tilton PF, Shaffer RD. Quantitation of the antimalarial agent, mefloquine, in blood, plasma and urine using high pressure liquid chromatography. J Pharm Sci. 1979;66:834-7.

Gschwend MH, Martin W, Erenmemisoglu A, Scherm M, Dilger C, Tamur U, et al. Pharmacokinetics and bioequivalence study of doxycycline capsules in healthy male subjects. Arzneimittelforschung. 2007;57:347-51.

Guentert TW, Coates PE, Upton RA, Combs DL, Riegelman S. Determination of quinidine and its major metabolites by high performance liquid chromatography. J Chromatogr. 1979;162:59-70.

Guevart E, Aguemon A. Deux hepatitis fulminantes survenues au cours d'un traitement curative par l'association artesunate-amodiaquine. [Two cases of fulminant hepatitis during curative treatment with an artesunate-amodiaquine combination]. Med Mal Infect. 2009;39:57-60.

Guilin Pharmaceuticals. Injectable artesun product information. 2011.

Gupta KC, Joshi JV, Desai NK, Sankoll GM, Chowdhary VN, Joshi UM, Chitalange S, Satoskar RS. Kinetics of chloroquine and contraceptive steroids in oral contraceptive users during concurrent chloroquine prophylaxis. Indian J Med Res. 1984;80:658-62.

Gurra R. The introduction of cinchona in the treatment of malaria. J Trop Med Hyg. 1977;80:122-35.

Gustafsson LL, Walker O, Alvan G. Disposition of chloroquine in man after single intravenous and oraldoses. Br J Clin Pharmacol. 1983a;15:471-9.

Gustafsson LL, Bergqvist Y, Ericsson O, Larsson M, Rombo L. Pitfalls in the measurement of chloroquine concentrations. Lancet. 1983b;I:126.

Gustafsson LL, Rombo L, Alvan G, Bjorkman A, Lind M, Walker O. On the question of dose-dependent chloroquine elimination of a single oral dose. Clin Pharmacol Ther. 1983c;34(3):383-5.

Gustafsson LL, Lindstrom B, Grahnen A, Alvan E. Chloroquine excretion following malaria prophylaxis. Br J Clin Pharmacol. 1987;24:221-4.

Hall AP, Peters W. The treatment of severe falciparum malaria. BMJ. 1985;291:1146-7.

Hall AP, Czerwinski AW, Madonia EC, Evensen KL. Human plasma and urine quinine levels following tablets, capsules and intravenous infusion. Clin Pharmacol Ther. 1973;14:580-5.

Hall AP, Doberstyn EB, Karnchanachetanee C, Samranramruakit S, Laixuthai B, Pearlman EJ, Lampe RM, Miller CF, Phintuyothin P. Sequential treatment with quinine and mefloquine or quinine and mefloquine or quinine and pyrimethamine-sulfadoxine for falciparum malaria. BMJ. 1977;1:1626-8.

Halpaap B, Ndjave M, Paris M, Benakis A, Kremsner PG. Plasma levels of artesunate and dihydroartemisinin in children with Plasmodium falciparum malaria in Gabon after administration of 50-milligram artesunate suppositories. Am J Trop Med Hyg. 1998;58:365-8.
Hanboonkunupakarn B, Ashley EA, Jittamala P, Tarning J, Pukrittayakamee S, Hanpithakpong W, et al. An openlabel crossover study of primaquine and dihydroartemisinin-piperaquine pharmacokinetics in healthy adult Thai subjects. Antimicrob Agents Chemother. 2014;58:7340-6.

Hardy BG, Sador IT, Golden L, Lalka D, Schentag JJ. Effect of cimetidine on the pharmacokinetics and pharmacodynamics of quinidine. Am J Cardiol. 1983;52:172-5.

Harinasuta T. Introduction. In: Laothavon J, Harinasuta T, editors. Drug resistant malaria. Technical report series no. 1. Bangkok: Ruamtasana Co. Ltd.; 1991. p. 11-23.

Harinasuta T, Migasena T, Migasena S, Bunnag D. Chloroquine resistance in Plasmodium falciparum in Thailand. UNESSCO regional symposium on scientific knowledge of tropical parasites, Singapore, 1962, p. $148-153$.

Harinasuta T, Bunnag D, Wernsdorfer WH. A phase-II clinical trial of mefloquine in patients with chloroquineresistant falciparum malaria in Thailand. Bull World Health Organ. 1983;61:299-305.

Harinasuta T, Bunnag D, Lasserre R, Leimer R, Vanijanond S. Trials of mefloquine in vivax and mefloquine plus Fansidar ${ }^{\mathrm{R}}$ in falciparum malaria. Lancet. 1985; $1: 885-8$.

Harinasuta T, Bunnag D, Vanijanond P, Charoenlarp P, Suntharamai P, Sheth UK, Wernsdorfer WH. Mefloquine, sulfadoxine and pyrimethamine in the treatment of symptomatic falciparum malaria: a double-blind trial for determining the most effective dose. Bull World Health Organ. 1987;65(3):363-7.

Harinasuta T, Bunnag D, Lasserre R. Quinine-resistant Plasmodium falciparum treated with mefloquine. Southeast Asian J Trop Med Public Health. 1990;21(4):552-7.

Hartel G, Harjanne A. Comparison of two methods for quinidine determination and chromatographic analysis of the difference. Clin Chim Acta. 1968;23:289-94.

Hassan-Ali M, Bjorkman A, Ashton M. In vitro activity of artemisinin, its derivatives, and pyronaridine against different strains of Plasmodium falciparum. Trans R Soc Trop Med Hyg. 1990;84:635-7.

Hatton CS, Peto TE, Bunch C, Pasvol G, Russell SJ, Singer C, et al. Frequency of severe neutropenia associated with amodiaquine prophylaxis against malaria. Lancet. 1986; i(8478):411-4.

Hatz C, Soto J, Nothdurft HD, Zoller T, Weitzel T, Loutan L, et al. Treatment of acute uncomplicated falciparum malaria with artemether-lumefantrine in nonimmune populations: a safety, efficacy, and pharmacokinetic study. Am J Trop Med Hyg. 2008;78:241-7.

Hein TT, Arnold K, Vinh H. Comparison of artemisinin suppositories with intravenous artesunate and intravenous quinine in the treatment of cerebral malaria. Trans R Soc Trop Med Hyg. 1992;86:582-3.

Heinzmann P, Geschke R. Determination of the antimalarial mefloquine in human plasma by gas 
chromatography with electroncapture detector. J Chromatogr. 1984;311:411-7.

Hellgren U, Kihamia CM, Mahikwano LF, Bjorkman A, Eriksson O, Rombo L. Respone of Plasmodium falciparum to chloroquine treatment: relation to whole blood concentrations of chloroquine and desethylchloroquine. Bull World Health Organ. 1989a;67(2):197-202.

Hellgren U, Kihamia CM, Mahikwano LF, Bjorkman A, Eriksson O, Rombo L. Response of P. falciparum to chloroquine treatment: relation to whole blood concentrations of chloroquine and desethylchloroquine. Bull World Health Organ. 1989b;67(2):197-202.

Hellgren U, Angel VH, Berqvist Y, Arvidsson A, Segundo J, Fererogomey JS, Rombo R. Plasma concentrations of sulfadoxine-pyrimethamine and of mefloquine during regular long term malaria prophylaxis. Trans R Sox Trop Med Hyg. 1990;84:46-9.

Hellgren U, Berggren-Palme I, Bergqvist Y, Jerling M. Enantioselective pharmacokinetics of mefloquine during long-term intake of the prophylactic dose. $\mathrm{Br}$ J Clin Pharmacol. 1997;44:119-24.

Hendriksen IC, Maiga D, Lemnge MM, Mtove G, Gesase S, Reyburn H, et al. Population pharmacokinetic and pharmacodynamic properties of intramuscular quinine in Tanzanian children with severe falciparum malaria. Antimicrob Agents Chemother. 2013a;57:775-83.

Hendriksen IC, Mtove G, Kent A, Gesase S, Reyburn H, Lemnge MM, et al. Population pharmacokinetics of intramuscular artesunate in African children with severe malaria: implications for a practical dosing regimen. Clin Pharmacol Ther. 2013b;93:443-50.

Hennegvin C, Boure P, Halfon P. Agranulocytosis during treatment with mefloquine. Lancet. 1991;377:984.

Hess KM, Goad JA, Arguin PM. Intravenous artesunate for the treatment of severe malaria. Ann Pharmacother. 2010;44:1250-8.

Hidalgo K, Lyles A, Dean SR. A potential interaction between warfarin and atovaquone. Ann Pharmacother. 2011;45:e3.

Hiebsch RR, Raub TJ, Wattenberg BW. Primaquine blocks transport by inhibiting the formation of functional transport vesicles. Studies in a cellfree assay of protein transport through the Golgi apparatus. J Biol Chem. 1991;266:20323-8.

Hien TT, Tam DTH, Cuc NTK, Arnold K. Comparative effectiveness of artemisinin suppositories and oral quinine in children with acute falciparum malaria. Trans R Soc Trop Med Hyg. 1991;85:210-1.

Hien TT, Davis TM, Chuong LV, Ilett KF, Sinh DX, Phu $\mathrm{NH}$, et al. Comparative pharmacokinetics of intramuscular artesunate and artemether in patients with severe falciparum malaria. Antimicrob Agents Chemother. 2004;48:4234-9.

Hietala SF, Bhattarai A, Msellem M, Roshammar D, Ali AS, Stromberg J, et al. Population pharmacokinetics of amodiaquine and desethylamodiaquine in pediatric patients with uncomplicated falciparum malaria. J Pharmacokinet Pharmacodyn. 2007;34:669-86.

Hietala SF, Martensson A, Ngasala B, Dahlstrom S, Lindegardh N, Annerberg A, et al. Population pharmacokinetics and pharmacodynamics of artemether and lumefantrine during combination treatment in children with uncomplicated falciparum malaria in Tanzania. Antimicrob Agents Chemother. 2010;54:4780-8.

Hill DR, Baird JK, Parise ME, Lewis LS, Ryan ET, Magill AJ. Primaquine: report from CDC expert meeting on malaria chemoprophylaxis I. Am J Trop Med Hyg. 2006;75:402-15.

Hobson-Frohoch A, Edwards WTP. Separation of cinchona alkaloids by high-performance liquid chromatography. J Chromatogr. 1982;249:36-372.

Hockwald RS, Arnold J, Clayman OB, Aliving AS. Status of primaquine: IV. Toxicity of primaquine in Negroes. JAMA. 1952;149:1568.

Hoffman SL, Dimperders AJ, Campbel JR, Marwoto HA, Sukri N, Rustama D, Punjabi NH, Oetomo HS, Harun S, Heizmann P, Laughlin LW. RII and RIII resistance of Plamsodium falciparum to combination of mefloquine and sulfadoxine/pyrimethamine in Indonesia. Lancet. 1985;2:1039-40.

Hoglund RM, Adam I, Hanpithakpong W, Ashton M, Lindegardh N, Day NP, et al. A population pharmacokinetic model of piperaquine in pregnant and nonpregnant women with uncomplicated Plasmodium falciparum malaria in Sudan. Malar J. 2012;11:398.

Holford MHG, Coates P, Guentert TW, Riegelman S, Shener L. The effect of quinidine and its metabolites on the electrocardiogram and systolic time intervals: concentration effect relationships. Br J Clin Pharmacol. 1981;11:187-95.

Hombhanje FW, Hwaihwanje I, Tsukahara T, Saruwatari J, Nakagawa M, Osawa H, et al. The disposition of oral amodiaquine in Papua New Guinean children with falciparum malaria. $\mathrm{Br} \mathrm{J}$ Clin Pharmacol. 2005;59:298-301.

Hoppe HC, van Schalkwyk DA, Wiehart UI, Meredith SA, Egan J, Weber BW. Antimalarial quinolines and artemisinin inhibit endocytosis in Plasmodium falciparum. Antimicrob Agents Chemother. 2004;48:2370-8.

Horowitz H, Carbonaro CA. Inhibition of the Salmonella typhi oral vaccine strain, Ty21a, by mefloquine and chloroquine. J Infect Dis. 1992;166:1462-4.

Houin G, Brunner F, Nebout T, Cherfaoui M, Lagrue G, Tillement JP. The effects of chronic renal insufficiency on the pharmacokinetics of doxycycline in man. $\mathrm{Br}$ J Clin Pharmacol. 1983;16:245-52.

Houze P, De Eeynies A, Bard FJ, Benatard MF, Pays M. Simultaneous determination of chloroquine and its three metabolites in human plasma, whole blood and urine by ionpair high performance liquid chromatography. J Chromatogr. 1992;574(2):305-12.

Howes RE, Battle KE, Satyagraha AW, Baird JK, Hay SI. G6PD deficiency: global distribution, genetic 
variants and primaquine therapy. Adv Parasitol. 2013;81:133-201.

Huang L, Parikh S, Rosenthal PJ, Lizak P, Marzan F, Dorsey $\mathrm{G}$, et al. Concomitant efavirenz reduces pharmacokinetic exposure to the antimalarial drug artemether-lumefantrine in healthy volunteers. J Acquir Immune Defic Syndr. 2012;61:310-6.

Hufford CD, Clark AM, Qunones IN, Baker JK, McChesney JD. Microbial metabolism studies on the major microbial and mammalian metabolite of primaquine. J Pharm Sci. 1983;72(1):92-4.

Hung TY, Davis TM, llett KF, Karunajeewa H, Hewitt S, Denis MB, et al. Population pharmacokinetics of piperaquine in adults and children with uncomplicated falciparum or vivax malaria. Br J Clin Pharmacol. 2001;57:253-62.

Hussin Z, Eaves CJ, Hutchinson DB, Canfield DJ. Population pharmacokinetics of proguanil in patients with acute $P$. falciparum malaria after combined therapy with atovaquone. Br J Clin Pharmacol. 1996;42:589-97.

Ibraim KE, Fell AF. Separation of chloroquine enantiomers by high-performance liquid chromatography. J Pharm Biomed Anal. 1990;8(5):449-52.

Ilett KF, Batty KT, Powell SM, Binh TQ, Thu Le TA, Phuong HL, et al. The pharmacokinetic properties of intramuscular artesunate and rectal dihydroartemisinin in uncomplicated falciparum malaria. $\mathrm{Br} \mathrm{J}$ Clin Pharmacol. 2002;53:23-30.

Inaba T, Tyndale RE, Mahon WA. Quinidine: potent inhibition of sparteine and debrisoquine oxidation in vivo. Br J Clin Pharmacol. 1986;22:199-200.

Ittarat W, Looareesuwan S, Pootrakul P, Sumpunsirikul P, Vattanavibool P, Meshnick SR. Effects of alphathalassemia on pharmacokinetics of the antimalarial agent artesunate. Antimicrob Agents Chemother. 1998;42:2332-5.

Jacobs F, Nicolaos G, Prieur S, Brivet F. Quinine dosage may not need to be reduced during continuous venovenous hemodiafiltration in severe anuric malaria. Clin Infect Dis. 2004;39:288-9.

Jamaludin A, Mohamad M, Navaratnam V, Selliah K, Tan SC, Wernsdorfer WH, Yuen KH. Relative bioavailability of the hydrochloride, sulphate and ethylcarbonate salts of quinine. Br J Clin Pharmacol. 1988;25:261-3.

Janssens B, van Herp M, Goubert L, Chan S, Uong S, Nong S, et al. A randomized open study to assess the efficacy and tolerability of dihydroartemisininpiperaquine for the treatment of uncomplicated falciparum malaria in Cambodia. Tropical Med Int Health. 2007;12:251-9.

Jha S, Kumar R, Kumar R. Mefloquine toxicity presenting with polyneuropathy - a report of two cases in India. Trans R Soc Trop Med Hyg. 2006;100:594-6.

Jiang JB, Li GQ, Guo WB, Kong YKC, Arnold $\mathrm{K}$. Antimalarial activity of mefloquine and qinghaosu. Lancet. 1982;2:285-8.

Jullien V, Ogutu B, Juma E, Carn G, Obonyo C, Kiechel JR. Population pharmacokinetics and pharmacodynamic considerations of amodiaquine and desethylamodiaquine in Kenyan adults with uncomplicated malaria receiving artesunate-amodiaquine combination therapy. Antimicrob Agents Chemother. 2010;54:2611-7.

Juma FD, Ogeto JO. Mefloquine disposition in normals and in patients with severs Plasmodium falciparum malaria. Eur J Drug metabol. 1989;14(1):15-7.

Kakuda TN, DeMasi R, van Delft Y, Mohammed P. Pharmacokinetic interaction between etravirine or darunavir/ritonavir and artemether/lumefantrine in healthy volunteers: a two-panel, two-way, two-period, randomized trial. HIV Med. 2013;14:421-9.

Kapetanovic IM, Digiovanni JD, Bartosevich J, Melendez V, von Brewdow J, Heiffer M. Analysis of the antimalarial mefloquine in blood and plasma using high performance liquid chromatography. J Chromatogr. 1983a;277:209-15.

Kapetanovic IM, Digiovanni JD, Bartosevich J, Melendez V, von Bredow J, Heiffer M. Analysis of the antimalarial mefloquine in blood and plasma using high performance liquid chromatography. J Chromatogr. 1983b;277:209-15.

Karbwang J, Harinasuta T. Distribution of drug resistance. Chemotherapy of malaria in Southeast Asia. Bangkok: Ruamtasana Co. Ltd.; 1992a. p. 47-72.

Karbwang J, Harinasuta T. Distribution of drug resistance. In: Karbwang J, Harinasuta T, editors. Chemotherapy of Malaria in Southeast Asia. Bangkok: Ruamtasana Co. Ltd.; 1992b. p. 48-72.

Karbwang J, Harinasuta T. Malaria parasites and clinical manifestation. In: Chemotherapy of malaria in Southeast Asia. Bangkok: Ruamtasana Co. Ltd.; 1992c. p. 19-39.

Karbwang J, Harinasuta T. Management of acute uncomplicated malaria. In: Chemotherapy of malaria in Southeast Asia. Bangkok: Ruamtasana Co. Ltd.; 1992d. p. 73-97.

Karbwang J, Na-Bangchang K. Clinical Pharmacology of antimalarials. In: Karbwang J, Back DJ, Breckenridge AM, editors. Clinical pharmacology in parasitic diseases. Thai Wattana Panich Press: Bangkok; 1992. p. 104-22.

Karbwang J, White NJ. Clinical pharmacokinetics of mefloquine. Clin Pharmacokinet. 1990;19(4):264-79.

Karbwang J, Bunnag D, Breckenridge AM, Back DJ. The pharmacokinetics of mefloquine when given alone or in combination with sulphadoxine and pyrimethamine in Thai male and female subjects. Eur J Clin Pharmacol. 1987a;32:173-7.

Karbwang J, Looareesuwan S, Phillips RE, Wattanagoon Y, Molyneux ME, Nagachinta B, Back DJ, Warrell DA. Plasma and whole blood mefloquine concentrations during treatment of chloroquineresistant falciparum malaria with the combination mefloquine-sulfadoxine-pyrimethamine. $\mathrm{Br} \mathrm{J}$ Clin Pharmacol. 1987b;23:477-81.

Karbwang J, Back DJ, Bunnag D, Breckenridge AM. A comparison of the pharmacokinetics of mefloquine in 
healthy Thai volunteers and in Thai patients with falciparum malaria. Eur J Clin Pharmacol. 1988a;35:677-80.

Karbwang J, Looareesuwan S, Back DJ, Migasena S, Bunnag D, Breckenridge AM. Effect of oral contraceptive steroids on the clinical course of malaria infection and the pharmacokinetics of mefloquine in Thai woman. Bull World Health Organ. 1988b;66:763-7.

Karbwang J, Molunto P, Na-Bangchang K, Bunnag D. Determination of mefloquine in biological fluids using high performance liquid chromatography. Southeast Asian J Trop Med Public Health. 1989a;20(1):55-60.

Karbwang J, Na-Bangchang K, Molunto P, Bunnag D. Determination of quinine and quinidine in biological fluids by high performance liquid chromatography. Southeast Asian J Trop Med Public Health. 1989b;20(1):65-79.

Karbwang J, Molunto P, Bunnag D, Harinasuta T. Plasma quinine levels patients with falciparum malaria when given alone or in combination with tetracycline with or without primaquine. Asian J Trop Med Public Health. 1991a;22(1):72-6.

Karbwang J, Na-Bangchang K, Back DJ, Bunnag D. Effects of ampicillin on mefloquine pharmacokinetics in Thai subjects. Eur J Clin Pharmacol. 1991b;40:631-3.

Karbwang J, Na-Bangchang K, Supapojana A, Bunnag D, Harinasuta T. Pharmacokinetics of prophylactic mefloquine in Thai healthy volunteers. Southeast Asian J Trop Med Public Health. 1991c;22(1):68-71.

Karbwang J, Na-Bangchang K, Thanavibul A, Bunnag D, Harinasuta T. Pharmacokinetics of mefloquine in treatment failure. Southeast Asian J Trop Med Public Health. 1991d;22(4):523-6.

Karbwang J, Na-Bangchang K, Bunnag D, Harinasuta T. Pharmacokinetics and pharmacodynamics of mefloquine in patients with uncomplicated malaria. Bull World Health Organ. 1991e;69(2):207-12.

Karbwang J, Bunnag D, Harinasuta T, Chittamas S, Berth J, Druilhe P. Pharmacokinetic of quinine, quinidine and cinchonine when given as combination. Southeast Asian J Trop Med Public Health. 1992a;23(4):773-6.

Karbwang J, Na-Bangchang K, Thanavibul A, Bunnag D, Chongsuphajaisiddhi T, Harinasnta T. Comparison of oral artemether and mefloquine in acute uncomplicated falciparum malaria. Lancet. 1992b;340:1245-8.

Karbwang J, Sukontason K, Rimchala W, Namsiripongpan W, Tin T, Auprayoon P, Tumsupapong S, Bunnag D, Harinasuta T. Preliminary report: a comparative clinical trial of artemether and quinine in severe falciparum malaria. Southeast Asian J Trop Med Public Health. 1992c;23(4):768-72.

Karbwang J, Na-Bangchang K, Back DJ, Bunnag D, Rooney W. Effect of tetracycline on mefloquine pharmacokinetics in Thai males. Eur J Clin Pharmacol. 1992d;43:567-9.
Karbwang J, Na-Bangchang K, Thanavibul A, Back DJ, Bunnag D. Pharmacokinetics of mefloquine in the presence of primaquine. Eur $\mathrm{J}$ Clin Pharmacol. 1992e;42:559-60.

Karbwang J, Na-Bangchang K, Thanavibul A, Bunnag D, Harinasuta T. Comparison of oral artemether and mefloquine in acute uncomplicated falciparum malaria. Lancet. 1992f;340:1245-8.

Karbwang J, Davis TME, Looareesuwan S, Molunto P, Bunnag D, White NJ. A comparison of the pharmacokinetics and pharmacodynamic properties of quinine and quinidine in healthy Thai males. $\mathrm{Br} \mathrm{J}$ Clin Pharmacol. 1993a;35:265-71.

Karbwang J, Na-Bangchang K, Thimasarn K, Rooney W, Bunnag D, Harinasuta T. Mefloquine levels in patients with mefloquine resistant Plasmodium falciparum in the eastern part of Thailand. Southeast Asian J Trop Med Public Health. 1993b;24(2):226-9.

Karbwang J, Thanavibul A, Molunto P, Na-Bangchang $\mathrm{K}$. The pharmacokinetics of quinine in patients with hepatitis. Br J Clin Pharmacol. 1993c;35:444-6.

Karbwang J, Bangchang KN, Thanavibul A, Wattanakoon Y, Harinasuta T. Quinine toxicity when given with doxycycline and mefloquine. Southeast Asian J Trop Med Public Health. 1994a;25:397-400.

Karbwang J, Na-Bangchang K, Thanavibul A, Back DJ, Bunnag D, Harinasuta T. Pharmacokinetics of mefloquine when given alone and in combination with artesunate. Bull World Health Organ. 1994b;72(1):83-7.

Karbwang J, Na-Bangchang K, Thanavibul A, Bunnag D, Chongsuphajaisiddi T, Harinasuta T. Comparison of oral artesunate and quinine plus tetracycline in acute uncomplicated falciparum malaria. Bull World Health Organ. 1994c;72(2):233-8.

Karbwang J, Na-Bangchang K, Congpuong K, Molunto P, Thanavibul A. Pharmacokinetics and bioavailability of oral and intramuscular artemether. Eur $\mathrm{J}$ Clin Pharmacol. 1997;52:307-10.

Karbwang J, Na-Bangchang K, Tin T, Sukontason K, Rimchala W, Harinasuta T. Pharmacokinetics of intramuscular artemether in patients with severe falciparum malaria with or without acute renal failure. Br J Clin Pharmacol. 1998;45:597-600.

Karema C, Fanello CI, van Overmeir C, van Geertruyden JP, van Doren W, Ngamije D, et al. Safety and efficacy of dihydroartemisinin/piperaquine (Artekin) for the treatment of uncomplicated Plasmodium falciparum malaria in Rwandan children. Trans R Soc Trop Med Hyg. 2006;100:1105-11.

Karunajeewa HA, Ilett KF, Dufall K, Kemiki A, Bockarie M, Alpers MP, et al. Disposition of artesunate and dihydroartemisinin after administration of artesunate suppositories in children from Papua New Guinea with uncomplicated malaria. Antimicrob Agents Chemother. 2004;48:2966-72.

Karunajeewa HA, Reeder J, Lorry K, Dabod E, Hamzah J, Page-Sharp M, et al. Artesunate suppositories versus intramuscular artemether for treatment of severe 
malaria in children in Papua New Guinea. Antimicrob Agents Chemother. 2006;50:968-74.

Karunajeewa HA, Ilett KF, Mueller I, Siba P, Law I, PageSharp M, et al. Pharmacokinetics and efficacy of piperaquine and chloroquine in Melanesian children with uncomplicated malaria. Antimicrob Agents Chemother. 2008;52:237-43.

Karunajeewa HA, Salman S, Mueller I, Baiwog F, Gomorrai S, Law I, et al. Pharmacokinetics of chloroquine and monodesethylchloroquine in pregnancy. Antimicrob Agents Chemother. 2010;54:1186-92.

Katsenos S, Psathakis K, Nikolopoulou MI, Constantopoulos SH. Mefloquineinduced eosinophilic pneumonia. Pharmacotherapy. 2007;27:1767-71.

Kavanagh MK, Wyse DG, Mitchell LB, Gilhooly T, Gillis AM, Duff HJ. Contribution of quinidine metabolites to electrophysiologic responses in human subjects. Clin Pharmacol Ther. 1989;46:352-8.

Kawo NG, Msengi AE, Swai ABM, Orskov H, Alberti KGMM, McLarty DG. The metabolic effects of quinine in children with severe and complicated Plasmodium falciparum malaria in Dar es Salaam. Trans R Soc Trop Med Hyg. 1991;85:711-3.

Keating GM. Dihydroartemisinin/piperaquine: a review of its use in the treatment of uncomplicated Plasmodium falciparum malaria. Drugs. 2012;72:937-61.

Kester M, Karpa KD, Vrana KE. Elsevier's Integrated review pharmacology. 2nd ed. 2011. ISBN $1455727032,9781455727032$.

Ketrangsee S, Vijayakadga S, Yamokgul P, Jatapadma S, Thimasarn K, Rooney W. Comparative trial on the response of Plasmodium falciparum to halofantrine and mefloquine in Trat Province, Eastern Thailand. Southeast Asian J Trop Med Public Health. 1992;23:55-8.

Khaliq Y, Gallicano K, Tisdale C, Carignan G, Cooper C, McCarthy A. Pharmacokinetic interaction between mefloquine and ritonavir in healthy volunteers. $\mathrm{Br}$ J Clin Pharmacol. 2001;51:591-600.

Kim KA, Park JY, Lee JS, Lim S. Cytochrome P450 2C8 and CYP3A4/5 are involved in chloroquine metabolism in human liver microsomes. Arch Pharm Res. 2003;26:631-7.

Kim YR, Kuh HJ, Kim MY, Kim YS, Chung WC, Kim SI, et al. Pharmacokinetics of primaquine and carboxyprimaquine in Korean patients with vivax malaria. Arch Pharm Res. 2004;27:576-80.

Klasco RK, editor. DRUGDEX ${ }^{\circledR}$ System. Thomson Micromedex, Greenwood Village, Colorado.

Klayman DL. Qinghaosu (artemisinine): an antimalarial drug from China. Science. 1985;228:1049-55.

Klayman DL, Lin AJ, Acton N, Scovill JP, Hoch JM, Milhous WK, Theoharides AD. Isolation of artemisinin (Qinghaosu) from Artemisia annua growing in the United States. J Nat Prod. 1984;47(4):715-7.

Klayman DL, Ager AL, Fleckenstein L, Lin AI. Transdermal artelinic acid: an effective treatment for Plasmodium berghei-infected mice. Am J Trop Med Hyg. 1991;45(5):602-7.
Kloprogge F, Piola P, Dhorda M, Muwanga S, Turyakira E, Apinan S, et al. Population pharmacokinetics of lumefantrine in pregnant and nonpregnant women with uncomplicated Plasmodium falciparum malaria in Uganda. CPT Pharmacometriccs Syst Pharmacol. 2013; $13: \mathrm{e} 83$.

Kollaritsch H, Que JU, Kunz C, Wiedermann G, Herzog C, Cryz Jr SJ. Safety and immunogenicity of live oral cholera and typhoid vaccines administered alone or in combination with antimalarial drugs, oral polio vaccine, or yellow fever vaccine. $J$ Infect Dis. 1997;175:871-5.

Kollaritsch H, Karbwang J, Wiedermann G, Mikolasek A, Na-Bangchang K, Wernsdorfer WH. Mefloquine concentration profiles during prophylactic dose regimens. Wien Klin Wochenschr. 2000;112:441-7.

Konate AT, Yaro JB, Ouedraogo AZ, Diarra A, Gansane A, Soulama I, et al. Intermittent preventive treatment of malaria provides substantial protection against malaria in children already protected by an insecticidetreated bednet in Burkina Faso: a randomized, double-blind, placebocontrolled trial. PLoS Med. 2011;8:e1000408.

Koren G, Zarfin Y, Maresky D, Spiro TE, MacLeod SM. Pharmacokinetics of intravenous clindamycin in new born infants. Pediatric Pharmacol (New York). 1986;5:287-92.

Krafts K, Hempelmann E, Skorska-Stania A. From methylene blue to chloroquine: a brief review of the development of an antimalarial therapy. Parasitol Res. 2012;111:1-6.

Kredo T, Mauff K, Van der Walt JS, Wiesner L, Maartens G, Cohen $\mathrm{K}$, et al. Interaction between artemether-lumefantrine and nevirapine-based antiretroviral therapy in HIV-1-infected patients. Antimicrob Agents Chemother. 2011;55:5616-23.

Kremsner PG, Taylor T, Issifou S, Kombila M, Chimalizeni Y, Kawaza K, Bouyou Akotet MK, Duscha M, Mordmüller B, Kösters K, Humberg A, Miller RS, Weina P, Duparc S, Möhrle J, Kun JF, Planche T, Teja-Isavadharm P, Simpson JA, Köhler C, Krishna S. A simplified intravenous artesunate regimen for severe malaria. J Infect Dis. 2012;205(2):312-9.

Krishna S, White NJ. Pharmacokinetics of quinine, chloroquine and amodiaquine. Clinical implications. Clin Pharmacokinet. 1996;30:263-99.

Krishna S, Nagaraja NV, Planche T, Agbenyega T, BedoAddo G, Ansong D, et al. Population pharmacokinetics of intramuscular quinine in children with severe malaria. Antimicrob Agents Chemother. 2001a;45:1803-9.

Krishna S, Planche T, Agbenyega T, Woodrow C, Agranoff D, Bedu-Addo G, et al. Bioavailability and preliminary clinical efficacy of intrarectal artesunate in Ghanaian children with moderate malaria. Antimicrob Agents Chemother. 2001b;45:509-16.

Krogstad DJ, Gluzman IY, Kyle DE. Efflux of chloroquine from Plasmodium falciparum: mechanism of chloroquine resistance. Science. 1987;238:1283-5. 
Krotoski WA. Discovery of the hypnozoite and a new theory of malaria relapse. Trans R Soc Trop Med Hyg. 1985;79:1-11.

Krudsood S, Wilairatana P, Vannaphan S, Treeprasertsuk S, Silachamroon U, Phomrattanaprapin W, et al. Clinical experience with intravenous quinine, intramuscular artemether and intravenous artesunate for the treatment of severe malaria in Thailand. Southeast Asian J Trop Med Public Health. 2003;34:54-61.

Krudsood S, Looareesuwan S, Tangpukdee N, Wilairatana P, Phumratanaprapin W, Leowattana W, et al. New fixed-dose artesunate-mefloquine formulation against multidrug-resistant Plasmodium falciparum in adults: a comparative phase IIb safety and pharmacokinetic study with standard-dose nonfixed artesunate plus mefloquine. Antimicrob Agents Chemother. 2010;54(9):3730-7.

Krudsood S, Looareesuwan S, Wilairatama P, Leowattana W, Tangpukdee N, Chalermrut K, et al. Effect of artesunate and mefloquine in combination on the Fridericia corrected QT intervals in Plasmodium falciparum infected adults from Thailand. Tropical Med Int Health. 2011;16:458-65.

Kulkarni SP, Shah SR, Kadam PP, Sridharan K, Hase NK, Shetty PP, et al. Pharmacokinetics of single-dose primaquine in patients with chronic kidney dysfunction. Indian J Pharm. 2013;45:330-3.

Kuye JO, Wilson MJ, Walle T. Gas chromatographic analysis of chloroquine after a unique reaction with chloroformates. J Chromatogr. 1983;272:307-13.

Lamorde M, Byakika-Kibwika P, Mayito J, Nabukeera L, Ryan M, Hanpithakpong W, et al. Lower artemether, dihydroartemisinin and lumefantrine concentrations during rifampicin-based tuberculosis treatment. AIDS. 2013;27:961-5.

Lancaster DL, Adio RA, Tai KK, Simooya OO, Broadhead GD, Tucker GT, Lennard MS. Inhibition of metoprolol metabolism by chloroquine and other antimalarial drugs. J Pharm Pharmacol. 1989;42:267-71.

Laothavorn P, Karbwang J, Na-Bangchang K, Bunnag D, Harinasuta T. Effect of mefloquine on electrocardiographic changes in uncomplicated falciparum malaria patients. Southeast Asian J Trop Med Public Health. 1992;23(1):51-4.

Le Jouan M, Jullien V, Tetanye E, Tran A, Rey E, Treluyer $\mathrm{JM}$, et al. Quinine pharmacokinetics and pharmacodynamics in children with malaria caused by Plasmodium falciparum. Antimicrob Agents Chemother. 2005;49:3658-62.

Leclercq R. Mechanisms of resistance to macrolides and lincosamides: nature of the resistance elements and their clinical implications. Clin Infect Dis. 2002;34(4):482-92.

Lee IS, Hufford CD. Metabolism of antimalarial sesquiterpene lactones. Pharmacal Ther. 1990;48:345-55.

Lee BL, Tauber MG, Sadler B, Goldstein D, Chambers HF. Atovaquone inhibits the glucuronidation and increases the plasma concentrations of zidovudine. Clin Pharmacol Ther. 1996;59:14-21.

Lee SJ, McGready R, Fernandez C, Stepniewska K, Paw MK, Viladpai-nguen SJ, et al. Chloroquine pharmacokinetics in pregnant and nonpregnant women with vivax malaria. Eur J Clin Pharmacol. 2008;64:987-92.

Lee TM, Huang L, Johnson MK, Lizak P, Kroetz D, Aweeka F, et al. In vitro metabolism of piperaquine is primarily mediated by CYP3A4. Xenobiotica. 2012;42:1088-95.

Lefevre G, Bindschedler M, Ezzet F, Schaeffer N, Meyer I, Thomsen MS. Pharmacokinetic interaction trial between co-artemether and mefloquine. Eur J Pharm Sci. 2000;10:141-51.

Lefevre G, Looareesuwan S, Treeprasertsil S, Krudsood S, Silachamroon U, Gathmann I, et al. A clinical and pharmacokinetic trial of six doses of artemetherlumefantrine for multidrug-resistant Plasmodium falciparum malaria in Thailand. Am J Trop Med Hyg. 2001;64:247-56.

Lefevre G, Carpenter P, Souppart C, Schmidli H, Martin $\mathrm{JM}$, Lane $\mathrm{A}$, et al. Interaction trial between artemether-lumefantrine (Riamet) and quinine in healthy subjects. J Clin Pharmacol. 2002a;42:1147-58.

Lefevre G, Carpenter P, Souppart C, Schmidli H, McClean M, Stypinski D. Pharmacokinetics and electrocardiographic pharmacodynamics of artemether-lumefantrine (Riamet) with concomitant administration of ketoconazole in healthy subjects. $\mathrm{Br} \mathrm{J}$ Clin Pharmacol. 2002b;54:485-92.

Lelijveld J, Kortmann H. The eosin colour test of Dill and Glazko: a simple field test to detect chloroquine in urine. Bull World Health Organ. 1970;42:477-9.

Leonardi E, Gilvary G, White NJ, Nosten F. Severe allergic reactions to oral artesunate: a report of two cases. Trans R Soc Trop Med Hyg. 2001;95:182-3.

Levy M, Buskila D, Gladman DD, Urowitz MB, Koren G. Pregnancy outcome following first trimester exposure to chloroquine. Am J Perinatol. 1991;8:174-8.

Li T, Magnes LJ. Inhibition of liver alcohol-dehydrogenase by primaquine and 8-amino-6-methoxyquinoline compounds. Biochem Pharmacol. 1972;21:17-25.

Li G, Xing Bo G, Wen LW. A clinical study on the treatment of falciparum malaria with artemether intramuscular injection. J Med Chin. 1982a;62(5):293-4.

Li G, Guo X, Jin R, Wang Z, Jian H, Li Z. Clinical studies on treatment of cerebral malaria with qinghaosu and its derivatives. J Tradit Chin Med. 1982b;2:125-30.

Li GQ, Arnold K, Guo X-B, Jian HX, Fu LC. Randomised comparative study of mefloquine, qinghaosu and pyrimethamine-sulfadoxine in patients with falciparum malaria. Lancet. 1984a;2:1360-1.

Li Q, Guo X, Arnorld K, Jain H. Randomised comparative study of mefloquine, qinghaosu and pyrimethaminesulfadoxine in patients with falciparum malaria. Lancet. 1984b;15:1360-2.

Li GQ, Guo X, Jian H, Fu L. Observation on the efficacy of qinghaosu suppository in 100 cases of falciparum malaria. Lancet. 1985;1360-1. 
Li Q, Cantilena LR, Leary KJ, Saviolakis GA, Miller RS, Melendez V, et al. Pharmacokinetic profiles of artesunate after single in intravenous doses at $0.5,1$, 2,4 , and $8 \mathrm{mg} / \mathrm{kg}$ in healthy volunteers: a phase I study. Am J Trop Med Hyg. 2009;81:615-21.

Lin A, Klayman DL, Milhous WK. Antimalarial activity of a new water-soluble dihydroartemisinin derivatives. J Med Chem. 1985;30(11):2147-50.

Liotier J, Richard D, Deteix P, Coudore F, Souweine B. Quinine clearance during continuous veno-venous high-volume hemofiltration. Intensive Care Med. 2008;34:1925-6.

Liu QY, Horio Y, Mizuguchi H, Fujimoto K, Imamura I, Abe Y, Fukui H. Re-examination of $\left[{ }^{3} \mathrm{H}\right]$ mepyramine binding assay for histamine $\mathrm{H}_{1}$ receptor using quinine. Biochem Biophys Res Commun. 1992;189:378-84.

Lockey D, Bateman DN. Effect of oral activated charcoal on quinine elimination. $\mathrm{Br} \mathrm{J}$ Clin Pharmacol. 1989;27:92-4.

Loeb RF. Activity of a new antimalarial agent, chloroquine (SN 7618). J Am Med Assoc. 1946;130:1069-70.

Looareesuwan S, White NJ, Karbwang J, Turner RC, Phillips RE, Kietinun S, Rackow C, Warrell DA. Quinine and severe falciparum malaria in late pregnancy. Lancet. $1985 ; 2: 4-7$.

Looareesuwan S, White NJ, Chanthavanich P, Edwards GE, Cicholl DD, Bunch C, Warrell DA. Cardiovascular toxicity and distribution kinetics of intravenous chloroquine. $\mathrm{Br} \mathrm{J}$ Clin Pharmacol. 1986;22:33-6.

Looareesuwan S, White NJ, Warrell DA, Fertgol L, Dubach UG, Ranalder UB, Schwartz DE. Studies of mefloquine bioavailability and kinetics using a stable isotope technique: a comparison of Thai patients with falciparum malaria and healthy Caucasian volunteers. Br J Clin Pharmacol. 1987;24:37-42.

Looareesuwan S, Visavan C, Vanijanonta S, Wilairatana P, Suntharasamai P, Charoenlarp P, Arnold K, Kyle D, Canfield C, Webster K. Randomised trial of artesunate and mefloquine alone and in sequence for acute uncomplicated falciparum malaria. Lancet. 1992a;339:821-4.

Looareesuwan S, Kyle DE, Viravan C, Vanijanonta S, Wilairatana P, Charoenlarp P, Canfield CJ, Webster HK. Treatment of patients with recrudescent falciparum malaria with a sequential combination of artesunate and mefloquine. Am J Trop Med Hyg. 1992b;47(6):794-9.

Looareesuwan S, Oosterhuis B, Schilizzi BM, Sollie FA, Wilairatana P, Krudsood S, et al. Dose-finding and efficacy study for i.m. artemotil (betaarteether) and comparison with i.m. artemether in acute uncomplicated $P$. falciparum malaria. $\mathrm{Br} \mathrm{J}$ Clin Pharmacol. 2002;53:492-500.

Luo XD, Xie M. Sub-nanogram detection of dihydroartemisinin after chemical derivatization with diacetyldihydrofluorescein followed by highperformance liquid chromatography and uv absorption. Chromatographia. 1987;23(2):112-4.
Luzzi GA, Warrell DA, Barnes AJ, Dunbar EM. Treatment of primaquine-resistant Plasmodium vivax malaria. Lancet. 1992;340:310.

Manning L, Laman M, Page-Sharp M, Salman S, Hwaiwhanje I, Morep N, et al. Meningeal inflammation increases artemether concentrations in cerebrospinal fluid in Papua New Guinean children treated with intramuscular artemether. Antimicrob Agents Chemother. 2011;55:5027-33.

Mansor SM, Navaratnam V, Mohamad M, Hussein S, Kummar A, Jamudin A, Wernsdorfer WH. Single dose kinetic study of the triple combination mefloquine-sulfadoxine-pyrimethamine $\quad\left(\right.$ Fansime $\left.{ }^{\mathrm{R}}\right)$ in healthy male volunteers. Br J Clin Pharmacol. 1989;27:381-6.

Mansor SM, Taylor T, McGrath CS, Edwards G, Ward SA, Wirima JJ, Molyneux ME. The safety and kinetics of intramuscular quinine in Malawian children with moderately severe falciparum malaria. Trans R Soc Trop Med Hyg. 1990;84:482-7.

Mashaal H. Background of malaria epidemiology. Clin Malariol Jpn: SEAMIC/IMFJ. 1986;48:2-5.

Masimirembwa CM, Naik YS, Hasler JA. The effect of chloroquine on the pharmacokinetics and metabolism of praziquantel in rats and in humans. Biopharm Drug Dispos. 1994;15:33-43.

Massele AY, Kilewo C, Aden Abdi Y, Tomson G, Diwan VK, Ericsson O, et al. Chloquine blood concentrations and malaria prophylaxis in Tanzanian women during the second and third trimesters of pregnancy. Eur J Clin Pharmacol. 1997;52:299-305.

Maude RJ, Plewes K, Faiz MA, Hanson J, Charunwatthana P, Lee SJ, et al. Does artesunate prolong the electrocardiograph QT interval in patients with severe malaria? Am J Trop Med Hyg. 2009;80:126-32.

Mayxay M, Keomany S, Khanthavong M, Souvannasing P, Stepniewska K, Khomthilath T, et al. A phase III, randomized, non-inferiority trial to assess the efficacy and safety of dihydroartemisininpiperaquine in comparison with artesunate-mefloquine in patients with uncomplicated Plasmodium falciparum malaria in southern Laos. Am J Trop Med Hyg. 2010;83:1221-9.

Mberu EK, Ward SA, Winstanley PA, Watkins WM. Measurement of quinine in filter-paper absorbed blood by high performance liquid chromatography. J Chromatogr. 1991;570:180-4.

McChesney EW, Wyzen HS, McAuliff JP. The determination of 4-aminoquinoline antimalarials: reevaluation of the induced fluorescence method, with specific application to hydroxychloroquine analysis. J Am Pharm Assoc. 1965;45:640-5.

McCloskey KL. Quinine concentrations in blood following the consumption of gin and tonic preparations in a social setting. J Anal Toxical. 1978;2(3):110-2.

McElnay JC, Mukhtar HA, D’Arcy PF, Temple DJ, Collier PS. The effect of magnesium trisilicate and kaolin on the in vivo absorption of chloroquine. J Trop Med Hyg. 1982a;85:159-63. 
McElnay JC, Mukhtar HA, D’Arcy PF, Temple DJ. In vitro experiments on chloroquine and pyrimethamine absorption in the presence of antacid constituents or kaolin. J Trop Med Hyg. 1982b;85:153-8.

McElnay JC, Sidahmed AM, D'Arcy PF. Examination of the chloroquine-kaolin drug absorption interaction using the buccal partitioning model. J Clin Hosp Pharm. 1982c;7(4):269-73.

McEwen J. Artesunate-and amodiaquine-associated extrapyramidal reactions: a series of 49 cases in VigiBase. Drug Saf. 2012;35:667-75.

McGready R, Thwai KL, Cho T, Samuel, Looareesuwan S, White NJ, et al. The effects of quinine and chloroquine antimalarial treatments in the first trimester of pregnancy. Trans R Soc Trop Med Hyg. 2002;96:180-4.

McGready R, Stepniewska K, Edstein MD, Cho T, Gilveray G, Looareesuwan S, et al. The pharmacokinetics of atovaquone and proguanil in pregnant women with acute falciparum malaria. Eur J Clin Pharmacol. 2003a;59:545-52.

McGready R, Stepniewska K, Seaton E, Cho T, Cho D, Ginsberg A, et al. Pregnancy and use of oral contraceptives reduces the biotransformation of proguanil to cycloguanil. Eur J Clin Pharmacol. 2003b;59:553-7.

McGready R, Stepniewska K, Lindegardh N, Ashley EA, La Y, Singhasivanon P, et al. The pharmacokinetics of artemether and lumefantrine in pregnant women with uncomplicated falciparum malaria. Eur J Clin Pharmacol. 2006;62:1021-31.

McGredy R, Tan SO, Ashley EA, Pimanpanarak M, Viladpai-Nguen J, Phaiphun L, et al. A randomized controlled trial of artemether-lumefantrine versus artesunate for uncomplicated Plasmodium falciparum treatment in pregnancy. PLoS Med. 2008;5:e253.

McLachlan AM, Tett S, Day R, Cutler D. Interpretation of chloroquine pharmacokinetic data. Eur $\mathrm{J}$ Xlin Pharmacol. 1993;44:4407-8.

Meek SR, Doberstyn EB, Gauzere BA, Thanapanich C, Nordlander E. Treatment of falciparum malaria with quinine and tetracycline or combined mefloquine/ sulfadoxine/pyrimethamine on the Thai-Kampuchean border. Am J Trop Med Hyg. 1986;35:246-50.

Meel SR, Doberstyn EB, Gauzere BA, Thanapanich C, Nordlander E, Phunphaisan S. Treatment of falciparum malaria with quinine and tetracycline or combined mefloquine/sulfadoxine/pyrimethamine on the ThaiKampuchean Border. Am J Trop Med Hyg. 1986;35(2):246-50.

Mehra N, Bhasin VK. In vitro gametocytocidal activity of artemisinin and its derivatives on Plasmodium falciparum. Jpn J Med Sci Biol. 1993;46:37-43.

Melendez V, Peggins JO, Brewer TG, Theoharides AD. Determination of the antimalarial arteether and its demethylated metabolite in plasma by highperformance liquid chromatography with reductive electrochemical detection. J Pharm Sci. 1991;80:132-8.

Menan H, Faye O, Same-Ekobo A, Oga AS, Faye B, Kiki Barro CP, et al. Comparative study of the efficacy and tolerability of dihydroartemisinin-piperaquine-trimethoprim versus artemether-lumefantrine in the treatment of uncomplicated Plasmodium falciparum malaria in Cameroon, Ivory Coast and Senegal. Malar J. 2011;10:185.

Meyer FP, Specht H, Quednow B, Walther H. Influence of milk on the bioavailability of doxycycline-new aspects. Infection. 1989;17:245-6.

Mihaly GW, Ward SA, Edwards G, Nicholl DD, Orme MLE, Breckentidge AM. Pharmacokinetics of primaquine in man: identification of carboxylic acid derivative as a major plasma metabolite. $\mathrm{Br} \mathrm{J}$ Clin Pharmacol. 1984;17:441-6.

Mihaly GW, Ward SA, Edwards G, Nicholl DD, LçEorme M, Breckenridge AM. Pharmacokinetics of primaquine in man I. Studies of the absolute bioavailability and effects of dose size. Br J Clin Pharmacol. 1985;19:745-50.

Mihaly GW, Hyman KM, Smallwood RA. Highperformance liquid chromatographic analysis of quinine and its diastereoisomer quinidine. J Chromatogr. 1987;415:177-82.

Miller KD, Greenberg AE, Campbell CG. Treatment of severe malaria in the United States with a continuous infusion of quinine gluconate and exchange transfusion. N Engl J Med. 1989;321:65-70.

Miller RS, Li Q, Cantilena LR, Leary KJ, Saviolakis GA, Melendez V, et al. Pharmacokinetic profiles of artesunate following multiple intravenous doses of 2 , 4 , and $8 \mathrm{mg} / \mathrm{kg}$ in healthy volunteers: phase $1 \mathrm{~b}$ study. Malar J. 2012;11:255.

Mimica I, Fry W, Eckert G, Schwartz DE. Multiple dose kinetic study of mefloquine in healthy male volunteers. Chemotherapy. 1983;29:184-7.

Minzi OM, Marealle IA, Shekalaghe S, Juma O, Ngaimisi E, Chemba M, et al. Comparison of bioavailability between the most available generic tablet formulation containing artemether and lumefantrine on the Tan zanian market and the innovator's product. Malar J. 2013;12:174.

Mirghani RA, Hellgren U, Bertilsson L, Gustafsson LL, Ericsson O. Metabolism and elimination of quinine in healthy volunteers. Eur J Clin Pharmacol. 2003;59:423-7.

Mithwani S, Aarons L, Kokwaro GO, Majid O, Muchohi S, Edwards G, et al. Population pharmacokinetics of artemether and dihydroartemisinin following single intramuscular dosing of artemether in African children with severe falciparum malaria. $\mathrm{Br} \mathrm{J}$ Clin Pharmacol. 2004;57:146-52.

Moore DV, Lanier JE. Observations on two Plasmodium falciparum infections with an abnormal response to chloroquine. Am J Trop Med Hyg. 1961;10:5-9.

Moore BR, Salman S, Benjamin J, Page-Sharp M, Robinson LJ, Waita E, et al. Pharmacokinetic properties of single-dose primaquine in Papua New Guinean children: feasibility of abbreviated high-dose regimens for radical cure of vivax malaria. Antimicrob Agents Chemother. 2014;58:432-9. 
Morris CA, Onyamboko MA, Capparelli E, Koch MA, Atibu J, Lokomba V, et al. Population pharmacokinetics of artesunate and dihydroartemisinin in pregnant and non-pregnant women with malaria. Malar J. 2011;10:114.

Morris CA, Lopez-Lazaro L, Jung D, Methaneethorn J, Duparc S, Borghini-Fuhrer I, Pokorny R, Shin CS, Fleckenstein L. Drug-drug interaction analysis of pyronaridine/artesunate and ritonavir in healthy volunteers. Am J Trop Med Hyg. 2012;86(3):489-95.

Most H, London Im, Kane CA, Lavietes PH, Schroeder EF, Haymanm JM. Chloroquine for treatment of acute attacks of vivax malaria. JAMA. 1946;131:963-7.

Mount DL, Churchill FC. Determination of mefloquine in blood, filter paper-absorbed blood and urine by 9-fluorenyl-methylchloroformate derivatization followed by liquid chromatography with fluorescence detection. J Chromatogr. 1991;564:181-3.

Mount DL, Nahlen BL, Patchen LC, Churchill FC. Fieldadapted method for high performance thinlayer chromatographic detection and estimation of chloroquine and desethylchloroquine in urine. J Chromatogr. 1987a;432:261-9.

Mount DL, Patchen LC, Williams SB, Churchill FC. Colorimetric and thin-layer chromatographic method for field assay of chloroquine and its metabolites in urine. Bull World Health Organ. 1987b;65:615-23.

Mount DL, Patchen LC, Churchill FC. Field-adapted method for high-performance thin-layer chromatographic detection and estimation of chloroquine in finger-stick blood. J Chromatogr. 1988;428:196-202.

Mu JY, Israilt ZH, Dayton PG. Studies of the disposition and metabolism of mefloquine HCL (WR 142, 490), a quinolinemethanol antimalarial in rat. Drug Metabol Disp. 1975;3:198-210.

Mulder B, Gazin P, Eggelte TA, Cot M. Increase of chloroquine resistance in vivo of Plasmodium falciparum over two years in Edea, South Cameroon. Trans R Soc Trop Med Hyg. 1992;86:376.

Muller AE, Mouton JW, Oostvogel PM, Dorr PJ, Voskuyl RA, DeJongh J, et al. Pharmacokinetics of clindamycin in pregnant women in the peripartum period. Antimicrob Agents Chemother. 2010;54:2175-81.

Munera Y, Hugues FC, Le Jeunne C, Pays JF. Interaction of thyroxine sodium with antimalarial drugs. BMJ. 1997;314:1593.

Murphy GS, Basri H, Purnomo Andersen EM, Bangs MJ, Mount DL, Gorden J, Lal AA, Purwokusumom AR, Harjosuwarno S, Sorensen K, Hoffmann SL. Vivax malaria resistant to treatment and prophylaxis with chloroquine. Lancet. 1993;341:96-100.

Murphy SA, Mberu E, Muhia D, English M, Crawley J, Waruiru C, et al. The disposition of intramuscular artemether in children with cerebral malaria; a preliminary study. Trans $\mathrm{R}$ Soc Trop Med Hyg. 1997;91:331-4.

Murray M. In vitro effects of quinoline derivatives on cytochrome P-450 aminopyrine $N$-demethylase activity in rat hepatic microsomes. Biochem Pharmacol. 1984a;33:3277-81.

Murray M. Invitro effects of quinine derivatives on cytochrome P-450 aminopyrine $N$-demethylase activity in rat hepatic microsomes. Biochem Pharmacol. 1984b;33:3277-81.

Mutabingwa TK, Muze K, Ord R, Briceno M, Greenwood $\mathrm{BM}$, Drakeley $\mathrm{C}$, et al. Randomized trial of artesunate+amodiaquine, sulfadoxinepyrimethamine+amodiaquine, chlorproguanil-dapsone and SP for malaria in pregnancy in Tanzania. PLoS One. 2009;4:e5138.

Mwebaza N, Jerling M, Gustafsson LL, Obua C, Waako P, Mahindi M, et al. Comparable lumefantrine oral bioavailability when co-administered with oilfortified maize porridge or milk in healthy volunteers. Basic Clin Pharmacol Toxicol. 2013;113:66-72.

Mwesigwa J, Parikh S, McGee B, German P, Drysdale T, Kalyango JN, et al. Pharmacokinetics of artemether-lumefantrine and artesunate-amodiaquine in children in Kampala, Uganda. Antimicrob Agents Chemother. 2010;54:52-9.

Mya OO, Tin A, Myein A. A controlled clinical trial of artemether and quinine in the treatment of falciparum malaria. Bur Med J. 1985;31(1):35.

Myint PT, Shwe T. The efficacy of artemether (qinghaosu) in Plasmodium falciparum and $P$. vivax in Burma. Southeast Asian J Trod Med Public Health. 1986;17:19-22.

Myint PT, Shwe T. A controlled clinical trial of artemether (qinghaosu derivative) versus quinine in complicated and severe falciparum malaria. Trans R Soc Trop Med Hyg. 1987;81:559-61.

Myint PT, Shwe T, Lin S. Clinical study of the treatment of cerebral malaria with artemether (qinghaosu derivative). Trans R Soc Trop Med Hyg. 1989;83:72.

Na-Bangchang K, Karbwang J, Davis TME, Looareesuwan S, Puakrittayakamee S, White NJ. Absorption kinetics of mefloquine in pregnant women with acute uncomplicated falciparum malaria. Br J Clin Pharmacol. 1989;29(1):149-52.

Na-Bangchang K, Karbwang J, Bunnag D, Harinasuta T. The effect of metoclopramide on mefloquine pharmacokinetics. Br J Clin Pharmacol. 1991;32(5):639.

Na-Bangchang N, Karbwang J, Back DJ. Primaquine metabolism by human liver microsomes: effect of other antimalarial drugs. Biochem Pharmacol. 1992a;44:587-90.

Na-Bangchang K, Karbwang J, Back DJ. Mefloquine metabolism by human liver microsomes: effect of other antimalarial drugs. Biochem Pharmacol. 1992b;43(9):1957-61.

Na-Bangchang K, Karbwang J, Banmairuroi V, Bunnag D, Harinasuta T. Mefloquine monitoring in acute uncomplicated malaria treated with Fansimef and Lariam. Southeast Asian J Trop Med Public Health. 1993;24(2):222-5.

Na-Bangchang K, Davis TM, Looareesuwan S, White NJ, Bunnag D, Karbwang J. Mefloquine pharmacokinetics 
in pregnant women with acute falciparum malaria. Trans R Soc Trop Med Hyg. 1994a;88:321-3.

Na-Bangchang $\mathrm{K}$, Karbwang J, Thomas CG, Thanavibul K, Sukontason K, Ward SA, Edwards G. Pharmacokinetics of artemether after oral administration to healthy Thai males and patients with acute uncomplicated falciparum malaria. $\mathrm{Br} \mathrm{J}$ Clin Pharmacol. 1994b;37(3):249-53.

Na-Bangchang K, Karbwang J, Davis TME, Looareesuwan S, Pukritayakamee S, White NJ. Absorption kinetics of mefloquine in pregnant patient with chloroquine resistant falciparum malaria. Trans R Soc Trop Med Hyg. 1994c;88:220-22.

Na-Bangchang K, Songsaeng W, Thanavibul A, Charoenlarp P, Karbwang J. Pharmacokinetics of primaquine in G6PD-deficient and G6PD-normal patients with vivax malaria. Trans R Soc Trop Med Hyg. 1994d;38(3):278-81.

Na-Bangchang K, Limpaibul L, Thanavibul A, Tan-Ariya$\mathrm{P}$, Karbwang J. The pharmacokinetics of chloroquine in healthy Thai subjects and patients with Plasmodium vivax malaria. Br J Clin Pharmacol. 1994;38:278-81.

Na-Bangchang K, Karbwang J, Tasanor U, Thanavibul A, Farkad E, Mull R. Pharmacokinetics of benflumetol given as a fixed combination artemether-benflumetol (CGP 56697) in Thai patients with uncomplicated falciparum malaria. Int $\mathrm{J}$ Clin Pharmacol Res. 1999a; 19:41-6.

Na-Bangchang K, Tan-Ariya P, Thanavibul A, Riengchainam S, Shrestha SB, Karbwang J. Pharmacokinetic and pharmacodynamic interactions of mefloquine and quinine. Int J Clin Pharmacol Res. 1999b;19:73-82.

Na-Bangchang K, Manyando C, Ruengweerayut R, Kioy D, Mulenga M, Miller GB, et al. The pharmacokinetics and pharmacodynamics of atovaquone and proguanil for the treatment of uncomplicated falciparum malaria in third-trimester pregnant women. Eur J Clin Pharmacol. 2005;61:573-82.

Nagagawa T, Higuchi T, Haslam JL, Shafferl RD, Mendenhall DW. GLC determination of whole blood antimalarial concentration. J Pharm Sci. 1979;68:718-21.

Nakae H, Asada S. Interaction of quinidine with phospholipids. I: effect on fluidity and permeability of phosphatidylcholine vesicles. Chem Pharm Bull. 1986;34(5):2169-72.

Nambozi M, Van Geertruyden JP, Hachizovu S, Chaponda M, Mukwamataba D, Mulenga M, et al. Safety and efficacy of dihydroartemisinin-piperaquine versus artemether-lumefantrine in the treatment of uncomplicated Plasmodium falciparum malaria in Zambian children. Malar J. 2011;10:50.

Navaratnam V, Ramanathan S, Wahab MS, Siew Hua G, Mansor SM, Kiechel JR, et al. Tolerability and pharmacokinetics of non-fixed and fixed combinations of artesunate and amodiaquine in Malaysian healthy normal volunteers. Eur J Clin Pharmacol. 2009;65:809-21.
Nayak VK, Desai NK, Kshirsagar NA, Bhandarkar SD, Satoskar RS. Antipyrine and doxycycline pharmacokinetics in patients with thyroid disorders. J Postgrad Med. 1991;37:5-8.

Ndifor AM, Howells RE, Ward SA. Chloroquine sensitivity of Plasmodium falciparum in vivo in a savanna town in Cameroon. Trans $\mathrm{R}$ Soc Trop Med Hyg. 1992;86:229-30.

Nealon C, Dzeing A, Muller-Romer U, Planche T, Sinou V, Kombila $\mathrm{M}$, et al. Intramuscular bioavailability and clinical efficacy of artesunate in Gabonese children with severe malaria. Antimicrob Agents Chemother. 2002;46:3933-9.

Neequaye J. In vivo chloroquine-resistant falciparum malaria in Western Africa. Lancet. 1986;1:153.

Neequaye J, Oforo-Adjei E, Ofori-Adjei D, Renner L. Comparative trial of oral versus intramuscular chloroquine in children with cerebral malaria. Trans $\mathrm{R}$ Soc Trop Med Hyg. 1991;85:718-22.

Neuvonen PJ, Penttila O. Interaction between doxycycline and barbiturates. Br Med J. 1974;i(5907):535-6.

Neuvonen PJ, Gothoni G, Hackman R, Bjorksten $\mathrm{K}$. Interference of iron with the absorption of tetracyclines in man. Br Med J. 1970;iv(5734):532-4.

Neuvonen PJ, Penttila O, Lehtovaara R, Aho K. Effect of antiepileptic drugs on the elimination of various tetracycline derivatives. Eur J Clin Pharmacol. 1975;9:147-54.

Neuvonen PJ, Penttila O, Roos M, Tirkkonen J. Effect of long-term alcohol consumption on the half-life of tetracycline and doxycycline in man. Int J Clin Pharmacol Biopharm. 1976;14:303-7.

Neuvonen PJ, Kivisto KT, Laine K, Pyykko K. Prevention of chloroquine absorption by activated charcoal. Hum Exp Toxicol. 1992;11(2):117-20.

Newton CRJC, Winstanley PA, Watkins WM, Mwangi IN, Waruiru CM, Mberu EK, Warn PA, Nevill CG, Marsh K. A single dose of intramuscular sulfadoxinepyrimethamine as an adjunct to quinine in the treatment of severe malaria: pharmacokinetics and efficacy. Trans R Soc Trop Med Hyg. 1993;87:207-10.

Newton P, Keeratithakul D, Teja-Isavadharm P, Pukrittayakamee S, Kyle D, White N. Pharmacokinetics of quinine and 3-hydroxyquinine in severe falciparum malaria with acute renal failure. Trans R Soc Trop Med Hyg. 1999;93:69-72.

Newton PN, Chaulet JF, Brockman A, Chierakul W, Dondorp A, Ruangveerayuth R, et al. Pharmacokinetics of oral doxycycline during combination treatment of severe falciparum malaria. Antimicrob Agents Chemother. 2005;49:1622-5.

Newton PN, Barnes KI, Smith PJ, Evans AC, Chierakul W, Ruangveerayuth $\mathrm{R}$, et al. The pharmacokinetics of intravenous artesunate in adults with severe falciparum malauria. Eur J Clin Pharmacol. 2006;62:1003-9.

Ngasala BE, Malmberg M, Carlsson AM, Ferreira PE, Petzold MG, Blessborn D, et al. Efficacy and effectiveness of artemether-lumefantrine after initial and repeated treatment in children $<5$ years of age with 
acute uncomplicated Plasmodium falciparum malaria in rural Tanzania: a randomized trial. Clin Infect Dis. 2011;52:873-82.

Ngouesse B, Basco LK, Ringwald P, Keundjian A, Blackett KN. Cardiac effects of amodiaquine and sulfadoxine-pyrimethamine in malaria-infected African patients. Am J Trop Med Hyg. 2001;65:711-6.

Nguyen DV, Nguyen QP, Nguyen ND, Le TT, Nguyen TD, Dinh DN, et al. Pharmacokinetics and ex vivo pharmacodynamic antimalarial activity of dihydroartemisininpiperaquine in patients with uncomplicated falciparum malaria in Vietnam. Antimicrob Agents Chemother. 2009;53:3534-7.

Nicholl DD, Edward SA, Orme ML'E, Breckenridge AM. The disposition of primaquine in the isolated perfused rat liver: stereoselective formation of the carboxylic acid metabolite. Biochem Pharmacol. 1987;36(20):3365-9.

Nilsen OG. Serum albumin and lipoproteins as the quinidine binding molecules in normal human sera. Biochem Pharmacol. 1976;25:1007-12.

Nora MV, Parkhurst GW, Thomas RW, Carson PE. Highperformance liquid chromatographic-electrochemical assay method for primaquine in plasma and urine. J Chromatogr. 1984;307:451-6.

Nosten F, Karbwang J, White NJ, Honeymoon, NaBangchang K, Harinasuta T. Mefloquine antimalarial prophylaxis in pregnancy: dose finding and pharmacokinetic study. Br J Clin Pharmacol. 1990;30(1):79-86.

Nosten F, ter Kuile F, Chongsuphajaisiddhi T, NaBangchang K, Karbwang J, White NJ. Mefloquine pharmacokinetics and resistance in children with acute falciparum malaria. $\mathrm{Br} \mathrm{J}$ Clin Pharmacol. 1991;31:556-9.

Nosten F, ter Kuile F, Maelankiri L, Chongsuphajaisiddhi T, Nopdonrattakoon L, Tangkitchot S, et al. Mefloquine prophylaxis prevents malaria during pregnancy: a double-blind, placebocontrolled study. J Infect Dis. 1994;169:595-603.

Novartis. Coartem product information. 2009.

Nyirjesy P, Kavasya T, Axelrod P, Fischer PR. Malaria during pregnancy: neonatal morbidity and mortality and the efficacy of chloroquine chemoprophylaxis. Clin Infect Dis. 1993;16:127-32.

Nyunt MM, Lu Y, El-Gasim M, Parsons TL, Petty BG, Hendrix CW. Effects of ritonavir-boosted lopinavir on the pharmacokinetics of quinine. Clin Pharmacol Ther. 2012;91:889-95.

Obua C, Hellgren U, Ntale M, Gustafsson LL, OgwalOkeng JW, Gordi T, et al. Population pharmacokinetics of chloroquine and sulfadoxine and treatment response in children with malaria: suggestions for an improved dose regimen. Br J Clin Pharmacol. 2008;65:493-501.

Ofori-Adjei D, Ericsson O, Lindstrom B, Hermansson J, Acljepon-Yamoah K, Sjoqvist F. Enantioselective analysis of chloroquine and desethylchloroquine after oral administration of racemic chloroquine. Ther Drug Monit. 1986a;8(4):457-61.
Ofori-Adjei D, Ericsson O, Linstorm B, Sjoqvist F. Protein binding of chloroquine enatiomers and desethyloroquine. $\mathrm{Br} \mathrm{J}$ Clin Pharmacol. 1986b;22(3):356-8.

Ogubona FA, Lawal AA, Onyeji CO. Saliva secretion of chloroquine in man. J Pharm Pharmacol. 1986;38(7):535-7.

Okonkwo CA, Coker HA, Agomo PU, Ogunbanwo JA, Mafe AG, Agomo CO, et al. Effect of chlorpheniramine on the pharmacokinetics of and response to chloroquine of Nigerian children with falciparum malaria. Trans R Soc Trop Med Hyg. 1999;93:306-11.

Okonkwom PO, Eta EI. Simultaneous determination of chloroquine and metronidazole in human biological fluid by high performance liquid chromatography. Life Sci. 1988;42(5):539-45.

Olatunde IA. Chloroquine concentrations in the skin of rabbits and man. Br J Pharmacol. 1971;43:335-40.

Onyamboko MA, Meshnick SR, Fleckenstein L, Koch MA, Atibu J, Lokomba V, et al. Pharmacokinetics and pharmacodynamics of artesunate and dihydroartemisinin following oral treatment in pregnant women with asymptomatic Plasmodium falciparum infections in Kinshasa DRC. Malar J. 2011;10:49.

Orme ML'E. Side effects of quinine and derivatives. Acta Leidensia. 1987;55:77-86.

Ouma P, Parise ME, Hamel MJ, Ter Kuile FO, Otieno K, Ayisi JG, et al. A randomized controlled trial of folate supplementation when treating malaria in pregnancy with sulfadoxine-pyrimethamine. PLoS Clin Trials. 2006;1:e28.

Pace JL. Psoriasis triggered by mefloquine. Skinmed. 2010;8:301-2.

Paintaud G, Alvan G, Ericsson O. The reproducibility of quinine bioavailability. $\mathrm{Br} \mathrm{J}$ Clin Pharmacol. 1993;35:305-7.

Paliwal JK, Gupta RC, Grover PK. Simultaneous determination of a new antimalarial agent, CDRI compound $80 / 53$, and its metabolite primaquine in serum by highperformance liquid chromatography. J Chromatogr. 1993;616:155-60.

Pang LW, Boudreau EF, Childs GE, Webster HK, Supanantalerk P. Failure of large-dose erythromycin in combination with a standard dose of chloroquine or quinine in the treatment of human falciparum malaria. Bull World Health Organ. 1985;63(4):739-43.

Pappaioanou M, Fishbein DB, Dreesen DW, Schwartz IK, Campbell GH, Sumner JW, et al. Antibody response to preexposure human diploidcell rabies vaccine given concurrently with chloroquine. $\mathrm{N}$ Engl J Med. 1986;314:280-4.

Parkhurst GW, Nora MV, Thomas RW, Carson PE. High performance liquid chromatographic-ultraviolet determination of primaquine and its metabolites in human plasma and urine. J Pharm Sci. 1984;73(9):1329-30.

Pasaribu AP, Chokejindachai W, Sirivichayakul C, Tanomsing N, Chavez I, Tjitra E, et al. A randomized comparison of dihydroartemisinin-piperaquine and 
artesunate-amodiaquine combined with primaquine for radical treatment of vivax malaria in Sumatera, Indonesia. J Infect Dis. 2013;208(11):1906-13. Epub 2013/ 08/09.

Patchen LC, Mount DL, Schwartz IK, Churchill FC. Analysis of filter-paper-absorbed, finger-stick blood samples for chloroquine and its major metabolite using highperformance liquid chromatography with fluorescence detection. J Chromatogr. 1983;273:81-9.

Patchen LC, Campbell CC, Williams SB. Neurologic reaction after a therapeutic dose of mefloquine. New Engl J Med. 1989;321:1415.

Pengsaa K, Sirivichayakul C, Na-Bangchang K, Thaiarporn I, Chaivisuth A, Wongsuwan A, et al. Life-saving rectal artesunate for complicated malaria in children. Southeast Asian J Trop Med Public Health. 2005;36:597-601.

Penttila O, Neuvonen PJ, Aho K, Lehtovaara R. Interaction between doxycycline and some antiepileptic drugs. $\mathrm{Br}$ Med J. 1974;ii(5917):470-2.

Peters W, Li ZL, Robinson BL, Warhurst DC. The chemotherapy of rodent malaria, XL the action of artemisinin and related sesquiterpenes. Ann Trop Med Parasitol. 1986;680(5):48-9.

Pfizer. Dalacin C product information. 2012.

Phillips RE, Looareesuwan S, Karbwang J, Warrell DA, White NJ, Kasemsarn P, Warhurst DC. Failure of chloroquine-erythromycin and chloroquinetetracycline combinations in treatment of chloroquineresistant falciparum malaria in eastern Thailand. Lancet. 1984;11:300-2.

Phillips RE, Warrell DA, White NJ, Looareesuwan S, Karbwang J. Intravenous quinidine for the treatment of severe falciparum malaria: clinical and pharmacokinetic studies. New Engl J Med. 1985;312(20):1273-8.

Phillips RE, Looareesuwan S, White NJ, Silamut K, Kietinun S, Warrell DA. Quinine pharmacokinetics and toxicity in pregnant and lactating women with falciparum malaria. $\mathrm{Br} \mathrm{J}$ Clin Pharmacol. 1986a;21:677-83.

Phillips RE, Looareesuwan S, White NJ, Silamut K, Kietinum S, Warrell DA. Quinine pharmacokinetics and toxicity in pregnant and lactating women with falciparum malaria. $\mathrm{Br} \mathrm{J}$ Clin Pharmacol. 1986b;21:677-83.

Phillips-Howard PA, Steffen R, Kerr L, Vanhauwere B, Schildknecht J, Fuchs E, et al. Safety of mefloquine and other antimalarial agents in the first trimester of pregnancy. J Travel Med. 1998;5:121-6.

Pinichpongse S, Doberstyn EB, Cullem JR, Yisunsri L, Thongsombun Y, Thimasarn K. An evaluation of five regimens for the outpatient therapy of falciparum malaria in Thailand 1980-81. Bull World Health Organ. 1982;60:907-12.

Prakongpan S, Sirimai S, Edwards GE, McGrath CS, White NJ. An improved formulation of chloroquine for intramuscular administration. J Pharm Pharmacol. 1989;41(10):726-8.
Prescott LF, Hamilton R, Heyworth R. Treatment of quinine overdosage with repeated oral charcoal. Br J Clin Pharmacol. 1989;27:95-7.

Price RN, Uhlemann AC, van Vugt M, Brockman A, Hutagalung R, Nair S, et al. Molecular and pharmacological determinants of the therapeutic response to artemether-lumefantrine in multidrug-resistant Plasmodium falciparum malaria. Clin Infect Dis. 2006;42:1570-7.

Price RN, Hasugian AR, Ratcliff A, Siswantoro H, Purba HL, Kenangalem E, et al. Clinical and pharmacological determinants of the therapeutic response to dihydroartemisinin-piperaquine for drug-resistant malaria. Antimicrob Agents Chemother. 2007;51:4090-7.

Pudney M, Gutteridge W, Zeman A, Dickins M, Woolley JL. Atovaquone and proguanil hydrochloride: a review of nonclinical studies. J Travel Med. 1999;6(Suppl 1): S8-12.

Pukrittayakamee S, Prakongpan S, Wanwimolruk S, Clemens R, Looareesuwan S, White NJ. Adverse effect of rifampin on quinine efficacy in uncomplicated falciparum malaria. Antimicrob Agents Chemother. 2003a;47:1509-13.

Pukrittayakamee S, Wanwimolruk S, Stepniewska K, Jantra A, Huyakorn S, Looareesuwan S, et al. Quinine pharmacokinetic-pharmacodynamic relationships in uncomplicated falciparum malaria. Antimicrob Agents Chemother. 2003b;47:3458-63.

Pukrittayakamee S, Tarning J, Jittamala P, Charunwatthana P, Lawpoolsri S, Lee SJ, et al. Pharmacokinetic interactions between primaquine and chloroquine. Antimicrob Agents Chemother. 2014;58:3354-9.

Pussard E, Verdier F, Blayo MC. Simultaneous determination of chloroquine, amodiaquine and their metabolites in human plasma, red blood cells, whole bold and urine by column liquid chromatography. J Chromatogr. 1986;374:111-8.

Pussard E, Barennes H, Daouda H, Clavier F, Sani AM, Osse M, et al. Quinine disposition in globally malnourished children with cerebral malaria. Clin Pharmacol Ther. 1999;65:500-10.

Pybus BS, Marcsisin SR, Jin X, Deye G, Sousa JC, Li Q, et al. The metabolism of primaquine to its active metabolite is dependent on CYP 2D6. Malar J. 2013;12:212.

Raghuram TC, Krishnaswamy K. Pharmacokinetics and plasma steady state levels of doxycycline in undernutrition. Br J Clin Pharmacol. 1982;14:785-9.

Raina RK, Bano G, Amla V, Kapoor V, Gupta KL. The effect of aspirin, paracetamol and analgin on pharmacokinetics of chloroquine. Indian J Physiol Pharmacol. 1993;37:229-31.

Ramharter M, Kurth FM, Belard S, Bouyou-Akotet MK, Mamfoumbi MM, Agnandji ST, et al. Pharmacokinetics of two paediatric artesunate mefloquine drug formulations in the treatment of uncomplicated falciparum malaria in Gabon. $\mathrm{J}$ Antimicrob Chemother. 2007;60:1091-6. 
Recht J, Ashley EA, White NJ. Safety of 8-aminoquinoline antimalarial medicines. Geneva: World Health Organization; 2014. http://www.who.int/malaria/publications/ atoz/9789241506977/en/.

Reijenga JC, Aben GVA, Lemmens AAG, Verheggen PEM, De Bruijn CHMM, Everaerts FM. Determination of quinine in beverages, pharmaceutical preparations and urine by isotachophareses. J Chemother. 1985;320:245-52.

Ridtitid W, Wongnawa M, Mahatthanatrakul W, Chaipol P, Sunbhanich M. Effect of rifampin on plasma concentrations of mefloquine in healthy volunteers. J Pharm Pharmacol. 2000;52:1265-9.

Ridtitid W, Wongnawa M, Mahatthanatrakul W, Raungsri N, Sunbhanich M. Ketoconazole increases plasma concentrations of antimalarial mefloquine in healthy human volunteers. J Clin Pharm Ther. 2005;30:285-90.

Rieckmann KH, Davis DR, Hutton DC. Plasmodium vivax resistance to chloroquine ? Lancet. 1989;2:1193-84.

Rijken MJ, McGready R, JuLLien V, Tarning J, Lindegardh N, Phyo AP, et al. Pharmacokinetics of amodiaquine and desethylamodiaquine in pregnant and postpartum women with Plasmodium vivax malaria. Antimicrob Agents Chemother. 2011a;55:4338-42.

Rijken MJ, McGready R, Phyo AP, Lindegardh N, Tarning J, Laochan N, et al. Pharmacokinetics of dihydroartemisinin and piperaquine in pregnant and nonpregnant women with uncomplicated falciparum malaria. Antimicrob Agents Chemother. 2011b;55:5500-6.

Rimchala P, Karbwang J, Sukontason K, Banmairuroi V, Molunto P, Na-Bangchang K. Pharmacokinetics of quinine in patients with chronic renal failure. Eur J Clin Pharmacol. 1996;49:497-501.

Riviere JH, Back DJ. Effect of mefloquine on hepatic drug metabolism in the rat: comparative study with primaquine. Biochem Pharmacol. 1985;34:567-71.

Riviere JH, Back DJ. Inhibition of ethinylestradiol and tolbutamide metabolism by quinoline derivatives in vivo. Chem Biol Interact. 1986a;59:301-8.

Riviere JH, Back DJ. Inhibition of ethinylestradiol and tolbutamide metabolism by quinoline derivatives in vitro. Chem Biol Interact. 1986b;59:301-8.

Roche. Lariam product information.

Rombo L, Bjorkman A, Sego E, Erwsson O. Whole blood concentrations of chloroquine and desethylchloroquine during and after treatment of adult patients infected with Plasmodium vivax, $P$. ovale or $P$. malariae. Trans R Soc Trop Med Hyg. 1986a;80:763-6.

Rombo L, Bjorkman A, Sego E, Ericsson O, Gustavsson L, Lindstrom B. Evaluation of three qualitative tests for detection of chloroquine in urine - Agreement with plasma concentrations determined with liquid chromatography. Ann Trop Med Parasitol. 1986b;80:293-8.

Roy L, Bannon P, Villeneuve JP. Quinine pharmacokinetics in chronic haemodialysis patients. $\mathrm{Br} \mathrm{J}$ Clin Pharmacol. 2002;54:604-9.
Ruangweerayut R, Looareesuwan S, Hutchinson D, Chauemung A, Banmairuroi V, Na-Bangchang $\mathrm{K}$. Assessment of the pharmacokinetics and dynamics of two combination regimens of fosmidomycinclindamycin in patients with acute uncomplicated falciparum malaria. Malar J. 2008;7:225.

Saano V, Paronen P, Peura P. Bioavailability of doxycycline from dissolved doxycycline hydrochloride tablets-comparison to solid form hydrochloride tablets and dissolved monohydrate tablets. Int $\mathrm{J}$ Clin Pharmacol Ther Toxicol. 1990;28:471-4.

Sabcharoen A, Chongsuphajaisiddhi T, Attanath P. Serum quinine concentrations following the initial dose in children with falciparum malaria. Southeast Asian J Trop Med Public Health. 1982;13(4):556-62.

Sabcharoen A, Chongsuphajaisiddhi T, Singhasivanon V, Chanthavanich $\mathrm{P}$, Attanath $\mathrm{P}$. In vivo and in vitro response to quinine and quinidine of Plasmodium falciparum. Bull World Health Organ. 1988;66(3):347-52.

Sabchareon A, Attanath P, Phanuaksook P, Chanthavanich P, Poonpanich Y, Mookmanee D, et al. Efficacy and pharmacokinetics of atovaquone and proguanil in children with multidrug-resistant Plasmodium falciparum malaria. Trans R Soc Trop Med Hyg. 1998;92:201-6.

Sadler B, Caldwell P, Scott J, Rogers M, Blum M. Drug interaction between rifampin and atovaquone in HIV+ asymptomatic volunteers (Poster A34). In: 35th Interscience Conference of Antimicrobial Agents and Chemotherapy. Washington, DC: American Society for Microbiology; 1995.

Saivin S, Houin G. Clinical pharmacokinetics of doxycycline and minocycline. Clin Pharmacokinet. 1988;15:355-66.

Salako LA, Ajayi FO. Distribution and urinary excretion of the desethylmetabolites of chloroquine in the rat. J Pharm Pharmacol. 1987;39(10):859-60.

Salako LA, Sowunmi A. Disposition of quinine in plasma, red blood cells and saliva after oral and intravenous administration to healthy adult Africans. Eur J Clin Pharmacol. 1992;42:171-4.

Salako LA, Walker O, Iyun AO. Pharmacokinetics of chloroquine in renal insufficiency. Afr $\mathrm{J}$ Med Sci. 1984;13:177-82.

Salako LA, Aderounmn AF, Walker O. Influence of route of administration on the pharmacokinetics of chloroquine and desethylchloroquine. Bull World Health Organ. 1987;65(1):47-50.

Salako LA, Sowumni A, Akinbami FO. Pharmacokinetics of quinine in African children children suffering from kwashiorkor. Br J Clin Pharmacol. 1989;28:197-201.

Salman S, Page-Sharp M, Griffin S, Kose K, Siba BM, Ilett $\mathrm{KF}$, et al. Population pharmacokinetics of artemether, Lumefantrine, and their respective metabolites in Papua New Guinean children with uncomplicated malaria. Antimicrob Agents Chemother. 2011;55:5306-13. 
Salman S, Page-Sharp M, Batty KT, Kose K, Griffin S, Siba PM, et al. Pharmacokinetic comparison of two piperaquine-containing artemisinin combination therapies in Papua New Guinean children with uncomplicated malaria. Antimicrob Agents Chemother. 2012;56:3288-97.

Sambol NC, Yan L, Creek DJ, McCormack SA, Arinaitwe E, Bigira V, et al. Population pharmacokinetics of piperaquine in young Ugandan children treated with dihydroartemisinin-piperaquine for uncomplicated malaria. Clin Pharmacol Ther. 2015;98 (1):87-95.

Saux MC, Mosser J, Pontagnier H, Leng B. Pharmacokinetics of doxycycline polyphosphate after oral multiple dosing in humans. Eur J Drug Metab Pharmacokinet. 1982;7:123-30.

Scarsi KK, Fehintola FA, Ma Q, Aweeka FT, Darin KM, Morse GD, et al. Disposition of amodiaquine and desethylamodiaquine in HIV-infected Nigerian subjects on nevirapine-containing antiretroviral therapy. J Antimicrob Chemother. 2014;69:1370-6.

Schapira A, Solomon T, Julien M, Macome A, Parmar M, Ruas I, Simao F, Streat E, Beschart B. Comparison of intramuscular and intravenous quinine for the treatment of severe and complicated malaria in children. Trans R Soc Trop Med Hyg. 1993;87:299-302.

Schiemann R, Coulaud JP, Bouchaud O. Seizures after antimalarial medication in previously healthy persons. J Travel Med. 2000;7:155-6.

Schmidt LH, Crosby R, Rasco JG, Vaughan D. Antimalarial activities of various 4-quinolinemetanols with special attention to WR 142-490 (mefloquine). Antimicrob Agents Chemother. 1978;13:1011-30.

Schneider C, Adamcova M, Jick SS, Schlagenhauf P, Miller MK, Rhein HG, Meier CR. Antimalarial chemoprophylaxis and the risk of neuropsychiatric disorders. Travel Med Infect Dis. 2013;11(2):71-80.

Schreder, H.W.R., Wolters, F.L., Vries, G. de. And Wetsteyn, J.C.F.M. Prospective in vitro study of chloroquine resistance of Plasmodium falciparum in Zambian under-fives. Trop Geogr Med 1993, 45(1), 15-17.

Schuurkamp GJ, Matango M, Napil J, Vergara RV. Malaria control and the development of the Ok Tedi Mining Project in the Star Mountains of Papua New Guinea: the initial construction phase. Papua New Guinea Med. 1987;30:27-38.

Schuurkamp GJ, Spicer PE, Kereu RK, Bulungol PK. A mixed infection of vivax and falciparum malaria apparently resistant to 4-aminoquinoline: a case report. Trans R Soc Trop Med Hyg. 1989;83:607-8.

Schuurkamp GJ, Spicer PE, Kereu RK, Bulungol PK, Reickmann KH. Chloroquine-resistant Plasmodium vivax in Papua New Guinea. Trans R Soc Trop Med Hyg. 1992;86:121-2.

Schwartz DE, Ranalder UB. Highly sensitie and specific determination of mefloquine in biological fluids using gas chromatography mass spectrometry with selected ion monitoring. Biochem Mass Spec. 1981;8:589-92.
Schwartz DE, Weber W, Richard-Lenoble D, Gentilini M. Kinetic Studies of mefloquine and of one of its metabolites, RO 215104, in the dog and in man. Acta Trop. 1980;37:238-42.

Schwartz DE, Eckert G, Hartmann D, Weber B, RichardLenoble D, Ekue JMK, Gentilinim M. Single dose kinetics of mefloquine in man. Chemotherapy. 1982;28:70-84.

Schwartz DE, Weidekamm E, Ranalder UB, Dubach UC, Forgo I, Webber B. Absence of pharmacokinetic interaction between Fansidar ${ }^{\mathrm{R}}$ and mefloquine. Trans R Soc Trop Med Hyg. 1986;80:1001-2.

Schwartz DE, Weidekam E, Mimica I, Heizman P, Portmann R. Multiple-dose pharmacokinetics of the antimalarial drug Fansimef (pyrimethamine+sulfadoxine+mefloquine) in healthy subjects. Chemotherapy. 1987a;33:1-8.

Schwartz DE, Eckert G, Ekue JMK. Urinary excretion of mefloquine and some of its metabolites in African volunteers at steady state. Chemotherapy. 1987b;33:305-8.

Schwartz IK, Lackritz EM, Patchen MS. Chloroquineresistant Plasmodium vivax from Indonesia. New Engl J Med. 1991;324:927.

Schwartz E, Potasman I, Rotenberg M, Almog S, Sadetzki $\mathrm{S}$. Serious adverse events of mefloquine in relation to blood level and gender. Am J Trop Med Hyg. 2001;65:189-92.

Seideman P, Albertioni F, Beck O, Eksborg S, Peterson C. Chloroquine reduces the bioavailability of methotrexate in patients with rheumatoid arthritis. A possible mechanism of reduced hepatotoxicity. Arthritis Rheum. 1994;37:830-3.

Shmuklarsky MJ, Boudreau EF, Pang LW, Smith JI, Schneider I, Fleckenstein L, et al. Failure of doxycycline as a causal prophylactic agent against Plasmodium falciparum malaria in healthy nonimmune volunteers. Ann Intern Med. 1994;120:294-9.

Shwe T, Myint PT, Htut Y, Myint W, Soe L. The effect of mefloquine artemether compared with quinine on patients with complicated falciparum malaria. Trans R Soc Trop Med Hyg. 1988;82:665-7.

Shwe T, Myint PT, Myint W, Htut Y, Soe L, Thwe M. Clinical studies on treatment of cerebral lmalaria with artemether and mefloquine. Trans R Soc Trop Med Hyg. 1989;83:489.

Silachamroon U, Krudsood S, Treeprasertsuk S, Wilairatana P, Chalearmrult K, Mint HY, et al. Clinical trial of oral artesunate with or without high-dose primaquine for the treatment of vivax malaria in Thailand. Am J Trop Med Hyg. 2003;69:14-8.

Silamut K, White NJ, Looareesuwan S, Warrell DA. Binding of quinine to plasmaproteins in falciparum malaria. Am J Trop Med Hyg. 1985;34(4):681-6.

Silamut K, Newton PN, Teja-Isavadharm P, Suputtamongkol Y, Siriyanonda D, Rasameesoraj M, et al. Artemether bioavailability after oral or intramuscular administration in uncomplicated falciparum 
malaria. Antimicrob Agents Chemother. 2003;47:3795-8.

Simpson JA, Watkins ER, Price RN, Aarons L, Kyle DE, White NJ. Mefloquine pharmacokinetic-pharmacodynamic models: implications for dosing and resistance. Antimicrob Agents Chemother. 2000;44:3414-24.

Simpson JA, Agbenyega T, Barnes KI, Di Perri G, Folb P, Gomes $\mathrm{M}$, et al. Population pharmacokinetics of artesunate and dihydroartemisinin following intrarectal dosing of artesunate in malaria patients. PLoS Med. 2006;3:e444.

Sindrup SH, Arendt-Nielsen L, Brosen K, Bjerring P, Angelo HR, Eriksen B, Gram LF. The effect of quinidine on the analgesic effect of codeine. Eur $\mathrm{J}$ Clin Pharmacol. 1992;42:587-92.

Singhasivanon V, Sabcharoen A, Attanath P, Chongsuphajaisiddhi T, Diquet B, Turk P. Pharmacokinetics of primaquine in healthy volunteers. Southeast Asian J Trop Med Public Health. 1991;22(4):529-33.

Single dose primaquine as a gametocytocide in Plasmodium falciparum malaria; updated WHO policy recommendation. Geneva: World Health Organization; 2012. http:// www.who.int/malaria/publications/atoz/who_pq policy_ recommendation/en/

Sinha S, Dua VK, Sharma VP. Malaria relapses and chloroquine resistance at the BHEL industrial complex, Hardwar, India. Trans R Soc Trop Med Hyg. 1989;83:606.

Sinou V, Malaika LT, Taudon N, Lwango R, Alegre SS, Bertaux L, et al. Pharmacokinetics and pharmacodynamics of a new ACT formulation: artesunate/ amodiaquine (TRIMALACT) following oral administration in African malaria patients. Eur J Drug Metab Pharmacokinet. 2009;34:133-42.

Sirivichayakul C, Sabchareon A, Pengsaa K, Thaiarporn I, Chaivisuth A, Na-Bangchang $K$, et al. Comparative study of the effectiveness and pharmacokinetics of two rectal artesunate/oral mefloquine combination regimens for the treatment of uncomplicated childhood falciparum malaria. Ann Trop Paediatr. 2007;27:17-24.

Smoak BL, Writer JV, Keep LW, Cowan J, Chantelois JL. The effects of inadvertent exposure of mefloquine chemoprophylaxis on pregnancy outcomes and infants of US Army servicewomen. J Infect Dis. 1997;176:831-3.

Smrkovski LL, Buck RL, Alcantara AK, Rodriguez ES, Uylanco CU. In vitro mefloquine resistant $P$. falciparum from the Philippines. Lancet. 1982;2:322.

Soentjens P, Delanote M, Van Gompel A. Mefloquineinduced pneumonitis. J Travel Med. 2006;13:172-4.

Sowunmi A. Disposition of oral quinine in African patients suffering from acute uncomplicated falciparum malaria. East Afr Med J. 1996;73:519-23.

Sowunmi A, Salako LA, Walker O, Ogundabunsi QAT. Clinical efficacy of mefloquine in children suffering from chloroquine resistant Plasmodium falciparum in Nigeria. Trans R Soc Trop Med Hyg. 1990;84:761-4.

Soyinka JO, Onyeji CO. Alteration of pharmacokinetics of proguanil in healthy volunteers following concurrent administration of efavirenz. Eur $\mathrm{J}$ Pharm Sci. 2010;39:213-8.

Soyinka JO, Onyeji CO, Omoruyi SI, Owolabi AR, Sarma PV, Cook JM. Effects of concurrent administration of nevirapine on the disposition of quinine in healthy volunteers. J Pharm Pharmacol. 2009;61:439-43.

Soyinka JO, Onyeji CO, Omoruyi SI, Owolabi AR, Sarma PV, Cook JM. Pharmacokinetic interactions between ritonavir and quinine in healthy volunteers following concurrent administration. $\mathrm{Br} \mathrm{J}$ Clin Pharmacol. 2010;69:262-70.

Srivastava IK, Vaidya AB. A mechanism for the synergistic antimalarial action of atovaquone and proguanil. Antimicrob Agents Chemother. 1999;43:1334-9.

Staehli Hodel EM, Csajka C, Ariey F, Guidi M, Kabanywanyi AM, Duong $S$, et al. Effect of single nucleotide polymorphisms in cytochrome $\mathrm{P} 450$ isoenzyme and $\mathrm{N}$-acetyltransferase 2 genes on the metabolism of artemisinin-based combination therapies in malaria patients from Cambodia and Tanzania. Antimicrob Agents Chemother. 2013a;57:950-8.

Staehli Hodel EM, Guidi M, Zanolari B, Mercier T, Duong S, Kabanywanyi AM, et al. Population pharmacokinetics of mefloquine, piperaquine and artemetherlumefantrine in Cambodian and Tanzanian malaria patients. Malar J. 2013b;12:235.

Stahel E, Druihe P, Gentilini M. Antagonism of chloroquine with other antimalarials. Trans R Soc Trop Med Hyg. 1988;82:221.

Staiger MA, Nguyen-Dinh P, Churchill FC. Sensitive highperformance liquid chromatographic analysis for chloroquine in body fluids. Applications to studies of drug resistance in Plasmodium falciparum. J Chromatogr. 1981;225:139-49.

Steketee RW, Mount DL, Patchen LC, Williams SB, Churchill FC, Roberts JM, Kaseje DC, Branding-Bennett AD. Field application of a colorimetric method of assaying chloroquine and its metabolites in urine. Bull World Health Organ. 1988;66:885-90.

Steketee RW, Wirima JJ, Slutsker L, Khoromana CO, Heymann DL, Breman JG. Malaria treatment and prevention in pregnancy: indications for use and adverse events associated with use of chloroquine or mefloquine. Am J Trop Med Hyg. 1996;55(Suppl 1):50-6.

Stepniewska K, Taylor W, Sirima SB, Ouedraogo EB, Ouedraogo A, Gansane A, et al. Population pharmacokinetics of artesunate and amodiaquine in African children. Malar J. 2009;8:200.

Stohrer JM, Dittrich S, Thongpaseuth V, Vanisaveth V, Phetsouvanh R, Phompida S, et al. Therapeutic efficacy of artemether-lumefantrine and artesunate-mefloquine for treatment of uncomplicated Plasmodium falciparum malaria in Luang Namtah Province, Lao People's Democratic Republic. Tropical Med Int Health. 2004;9:1175-83. 
Strother A, Fraser IM, Tilton B. Studies of the metabolism of the antimalarial agent primaquine. Abstract, sixth international congress of pharmacology, Helsinki 1975.

Strother A, Fraser IM, Allahyari R, Tilton BE. Metabolism of 8-aminoquinoline antimalarial agents. Bull World Health Organ. 1981;59(3):413-25.

Strother A, Allahyari R, Buchholz J, Fraser IM, Tilton B. In vitro Metabolism of the Antimalarial agent primaquine by mouse liver enzymes and identification of a methemoglobin-forming metabolite. Drug Metabol Disp. 1984;12:35-44.

Supanaranond W, Davis TM, Pukrittayakamee S, Silamut K, Karbwang J, Molunto P, et al. Disposition of oral quinine in acute falciparum malaria. Eur J Clin Pharmacol. 1991;40:49-52.

Svensson US, Alin H, Karlsson MO, Bergqvist Y, Ashton M. Population pharmacokinetic and pharmacodynamic modeling of artemisinin and mefloquine enantiomers in patients with falciparum malaria. Eur J Clin Pharmacol. 2002;58:339-51.

Tan KR, Magill AJ, Parise ME, Arguin PM. Doxycycline for malariachemoprophylaxis and treatment: report from the CDC expert meeting on malaria chemoprophylaxis. Am J Trop Med Hyg. 2011;84:517-31.

Tapanes RD, Ramos F, Avila J. Contribution to the determination of chloroquine in biological fluids. J Chromatogr. 1989;493:196-201.

Tarlov AR, Brewer GJ, Carson PE, Alving AS. Primaquine sensitivity glucose-6-phosphate dehydrogenase deficiency: an inborn error of metabolism of medical response. Trans R Soc Trop Med Hyg. 1962;109:209-34.

Tarning J. Piperaquine bioanalysis, drug metabolism and pharmacokinetics. Göteborg: Göteborg University; 2007.

Tarning J, Ashley EA, Lindegardh N, Stepniewska K, Phaiphun L, Day NP, et al. Population pharmacokinetics of piperaquine after two different treatment regimens with dihydroartemisinin-piperaquine in patients with Plasmodium falciparum malaria in Thailand. Antimicrob Agents Chemother. 2008;52:1052-61.

Tarning J, McGready R, Lindegardh N, Ashley EA, Pimanpanarak M, Kamanikom B, et al. Population pharmacokinetics of lumefantrine in pregnant women treated with artemether-lumefantrine for uncomplicated Plasmodium falciparum malaria. Antimicrob Agents Chemother. 2009;53:3837-46.

Tarning J, Chlotsiri P, Jullien V, Rijken MJ, Bergstrand M, Cammas $\mathrm{M}$, et al. Population pharmacokinetic and pharmacodynamic modeling of amodiaquine and desethylamodiaquine in women with Plasmodium vivax malaria during and after pregnancy. Antimicrob Agents Chemother. 2012a;56:5764-73.

Tarning J, Kloprogge F, Piola P, Dhorda M, Muwanga S, Turyakira E, et al. Population pharmacokinetics of artemether and dihydroartemisinin in pregnant women with uncomplicated Plasmodium falciparum malaria in Uganda. Malar J. 2012b;11:293.

Tarning J, Zongo I, Some FA, Rouamba N, Parikh S, Rosenthal PJ, et al. Population pharmacokinetics and pharmacodynamics of piperaquine in children with uncomplicated falciparum malaria. Clin Pharmacol Ther. 2012c;91:497-505.

Tarning JR, Rijken MJ, McGready R, Phyo AP, Hanpithakpong W, Day NPJ, et al. Population pharmacokinetics of dihydroartemisinin and piperaquine in pregnant and non-pregnant women with uncomplicated malaria. Antimicrob Agents Chemother. 2012d;56:1997-2007.

Tarning J, Kloprogge F, Dhorda M, Jullien V, Nosten F, White NJ, et al. Pharmacokinetic properties of artemether, dihydroartemisinin, lumefantrine, and quinine in pregnant women with uncomplicated Plasmodium falciparum malaria in Uganda. Antimicrob Agents Chemother. 2013;57:5096-103.

Tarning J, Lindegardh N, Lwin KM, Annerberg A, Kiricharoen L, Ashley E, et al. Population pharmacokinetic assessment of the effect of food on piperaquine bioavailability in patients with uncomplicated malaria. Antimicrob Agents Chemother. 2014;58:2052-8.

Taylor RB, Behren R, Moody RR, Wangboonskul J. Assay method for the simultaneous determination of proguanil, chloroquine and their major metabolites in biological fluids. J Chromatogr. 1990;527(2):490-7.

Taylor TE, Wills BA, Kazembe P, Chisale M, Wirima JJ, Ratsma EYEC, Molyneux ME. Rapid coma resolution with artemether in Malawian children with cerebral malaria. Lancet. 1993;341:661-2.

Tekete MM, Toure S, Fredericks A, Beavogui AH, Sangare CP, Evans A, et al. Effects of amodiaquine and artesunate on sulphadoxine-pyrimethamine pharmacokinetic parameters in children under five in Mali. Malar J. 2011;10:275.

Tenson T, Lovmar M, Ehrenberg M. The mechanism of action of macrolides, lincosamides and streptogramin $\mathrm{B}$ reveals the nascent peptide exit path in the ribosome. J Mol Biol. 2003;330:1005-14.

ter Kuile FO, Luxemburger C, Nosten F, Thwai KL, Chongsuphajaisiddhi T, White NJ. Predictors of mefloquine treatment failure: a prospective study of 1590 patients with uncomplicated falciparum malaria. Trans R Soc Trop Med Hyg. 1995a;89:660-4.

ter Kuile FO, Nosten F, Luxemburger C, Kyle D, TejaIsavatharm P, Phaipun L, et al. Mefloquine treatment of acute falciparum malaria: a prospective study of nonserious adverse effects in 3673 patients. Bull World Health Organ. 1995b;73:631-42.

Tett SE, Culter DJ. Apparent dose-dependence of chloroquine pharmacokinetics due to limited assay sensitivity and short sampling times. Eur J Clin Pharmacol. 1987;21(6):729-31.

Thaper MM, Ashton M, Lindegardh N, Bergqvist Y, Nivelius S, Johansson I, et al. Time-dependent pharmacokinetics and drug metabolism of atovaquone plus proguanil (Malarone) when taken as chemoprophylaxis. Eur J Clin Pharmacol. 2002;58:19-27.

The WorldWide Antimalarial Resistance Network DP Study Group. The effect of dosing regimens on the antimalarial efficacy of dihydroartemisinin-piperaquine: a pooled 
analysis of individual patient data. PLoS Med. 2013;10: e1001564.

Thimasarn K, Pinichpongse S, Malikul S, Rooney W, Tansophalaks S. Phase III double-blind comparative study of Fansimef ${ }^{\mathrm{R}}$ and Lariam $^{\mathrm{R}}$ for the curative treatment of Plasmodium falciparum infections in Thailand. Southeast Asian J Trop Med Public Health. 1990;21:404-11.

Thomas CG, Ward SA. Selective determination in plasma of artemether and its major metabolite dihydroartemisinin, by high performance liquid chromatography with ultraviolet detection. J Chromatogr. 1992;583:131-6.

Tin F, Hlaing N, Lasserre R. Single-dose treatment of falciparum malaria with mefloquine: field studies with different doses in semi-immune adults and children in Burma. Bull World Health Organ. 1982;60:913-7.

Tin F, Hlaing N, Tun T, Win S, Lasserre R. Falciparum malaria treated with a fixed combination of mefloquine, sulfadoxine and pyrimethamine: a field study in adults in Burma. Bull World Health Organ. 1985;63:727-30.

Tin F, Hlaing N, Lim AM, Win S, Lasserre R. Double-blind trial to find dose range using a fixed combination of mefloquine, sulfadoxine and pyrimethamine in falciparum malaria: a field study on adults in Burma. Trans R Soc Trop Med Hyg. 1987;81:910-2.

Tin S, Pe Than M, Ye H, Win M, Lin S. The effect of mefloquine-artemether compared with quinine on patients with complicated falciparum malaria. Trans R Soc Trop Med Hyg. 1988;82:665-6.

Tin S, Pe Than M, Win M, Ye H, Lin S, Min T. Clinical studies on treatment of cerebral malaria with artemether and mefloquine. Trans R Soc Trop Med Hyg. 1989;83:489.

Tine RC, Faye B, Sylla K, Ndiaye JL, Ndiaye M, Sow D, et al. Efficacy and tolerability of a new formulation of artesunate-mefloquine for the treatment of uncomplicated malaria in adult in Senegal: open randomized trial. Malar J. 2012;11:416.

Titulaer HA, Eling WM, Crommelin DJ, Peeters PA, Zuidema J. The parenteral controlled release of liposome encapsulated chloroquine in mice. J Pharm Pharmacol. 1990a;42(8):529-32.

Titulaer HAC, Zuidema J, Karger PA, Wetestyn JCFM, Lugt CB, Merkus FWHM. The pharmacokinetics of artemisinin after oral, intramuscular and rectal administration to human volunteers. J Pharm Pharmacol. 1990b;42:810-3.

Titulaer HAC, Zuidema J, Lugt CB. Formulation and pharmacokinetics of artemisinin and its derivatives. Int J Pharm. 1991;69:83-92.

Titus EO. Recent developments in the understanding of the pharmacokinetics and mechanism of action of chloroquine. Ther Drug Monit. 1989;11:369-79.

Tjitra E, Hasugian AR, Siswantoro H, Prasetyorini B, Ekowatiningsih R, Yusnita EA, et al. Efficacy and safety of artemisinin-naphthoquine versus dihydroartemisinin-piperaquine in adult patients with uncomplicated malaria: a multi-centre study in Indonesia. Malar J. 2012;11:153.

Todd GD, Hopperus Buma AP, Green MD, Jaspers CA, Lobel HO. Comparison of whole blood and serum levels of mefloquine and its carboxylic acid metabolite. Am J Trop Med Hyg. 1997;57:399-402.

Tommasi C, Bellagamba R, Tempestilli M, D'Avolio A, Gallo AL, Ivanovic J, et al. Marked increase in etravirine and saquinavir plasma concentrations during atovaquone/ proguanil prophylaxis. Malar J. 2011;10:141.

Treluyer JM, Roux A, Mugnier C, Flouvat B, Lagardere B. Metabolism of quinine in children with global malnutrition. Pediatr Res. 1996;40:558-63.

Trenholme GM, Williams RL, Desjardin RE, Frischer M, Carson PE, Rieckman KH, Canfield DJ. Mefloquine (WR142490) in treatment of human malaria. Science. 1975;190:792-4.

Trenholme GM, Williams RL, Rieckmann KH, Frischer H, Carson PE. Quinine disposition during malaria and during induced fever. Clin Pharmacol Ther. 1976;19:459-67.

Trigg I. Qinghaosu (artemisinin) as an antimalarial drug. Econ Med Plant Res. 1987;3:20-50.

Tuchinda S, Rucknagel DL, Na-Nakorn S, Wasi P. The Thai variant and the distribution of alleles of 6-phosphogluconate dehydrogenase and the distribution of glucose-6-phosphate dehydrogenase deficiency in Thailand. Biochem Genet. 1968;2:253-4.

Tulpule A, Krishnaswamy K. Effect of food on bioavailability of chloroquine. Eur J Clin Pharmacol. 1982;23:271-3.

Tulpule A, Krishnaswamy K. Chloroquine kinetics in the malnourished. Eur J Clin Pharmacol. 1983a;24(2):273-6.

Tulpule A, Krishnaswamy K. Effect of rice diet on chloroquine bioavailability. Drug Nutr Interact. 1983b;2(2):83-6.

Udry E, Bailly F, Dusmet M, Schnyder P, Lemoine R, Fitting JW. Pulmonary toxicity with mefloquine. Eur Respir J. 2001;18:890-2.

Ursing J, Kofoed PE, Rodrigues A, Blessborn D, ThoftNielsen R, Bjorkman A, et al. Similar efficacy and tolerability of double-dose chloroquine and artemether-lumefantrine for treatment of Plasmodium falciparum infection in Guinea-Bissau: a randomized trial. J Infect Dis. 2011;203:109-16.

Valecha N, Phyo AP, Mayxay M, Newton PN, Krudsood S, Keomany S, et al. An open-label, randomised study of dihydroartemisinin-piperaquine versus artesunate-mefloquine for falciparum malaria in Asia. PLoS One. 2010;5:e11880.

Valecha N, Mohanty S, Srivastava P, Sharma S, Tyagi P, Bergqvist Y, et al. Efficacy of artemether-lumefantrine in area of high malaria endemicity in india and its correlation with blood concentration of lumefantrine. Am J Trop Med Hyg. 2012;86:395-7.

van Agtmael MA, Cheng-Qi S, Qing JX, Mull R, van Boxtel DJ. Multiple dose pharmacokinetics of artemether in Chinese patients with uncomplicated 
falciparum malaria. Int $\mathrm{J}$ Antimicrob Agents. 1999a;12:151-8.

van Agtmael MA, Gupta V, van der Graaf CA, van Boxtel CJ. The effect of grapefruit juice on the time-dependent decline of artemether plasma levels in healthy subjects. Clin Pharmacol Ther. 1999b;66:408-14.

van Hensbroek MB, Kwiatkowski D, van den Berg B, Hoek FJ, van Boxtel CJ, Kager PA. Quinine pharmacokinetics in young children with severe malaria. Am J Trop Med Hyg. 1996;54:237-42.

Van Luin M, Van der Ende ME, Richter C, Visser M, Faraj D, Van der Ven A, et al. Lower atovaquone/ proguanil concentrations in patients taking efavirenz, lopinavir/ritonavir or atazanavir/ritonavir. AIDS. 2010;24:1223-6.

van Riemsdijk MM, Sturkenboom MC, Pepplinkhuizen L, Stricker BH. Mefloquine increases the risk of serious psychiatric events during travel abroad: a nationwide case-control study in the Netherlands. J Clin Psychiatry. 2005;66:199-204.

Vanhauwere B, Maradit H, Kerr L. Post-marketing surveillance of prophylactic mefloquine (Lariam) use in pregnancy. Am J Trop Med Hyg. 1998;58:17-21.

Venho VM, Salonen RO, Mattila MJ. Modification of the pharmacokinetics of doxycycline in man by ferrous sulphate or charcoal. Eur J Clin Pharmacol. 1978;14:277-80.

Viriyayudhakorn S, Thitiarchakul S, Nachaisit S, Ho PC, Wanwimolruk S. Pharmacokinetics of quinine in obesity. Trans R Soc Trop Med Hyg. 2000;94:425-8.

Von Jauch R, Greisser E, Oesterheltl G. Metabolism von Ro 21-5988 (mefloquine) bei der ratte. Arzneirnforsch Drug Res. 1980;30:60-7.

Vozeh S, Uematsu T, Guentert WT, Ha RH, Follath F. Kinetics and electrocardiographic changes after oral 3-OH-quinidine in healthy subjects. Clin Pharmacol Ther. 1985;37:575-81.

Walker O, Dawodu AH, Adeyokunnu AA, Salako LA, Alvan G. Plasma chloroquine and desethyl chloroquine in children during and after chloroquine treatment for malaria. Br J Clin Pharmacol. 1983;16:701-5.

Walker O, Dawodu AH, Salako LA, Alvan G, Johnson AO. Single dose disposition of chloroquine in kwashiorkor and normal children-evidence for decreased a bsorption in kwashiorkor. $\mathrm{Br} \mathrm{J}$ Clin Pharmacol. 1987;23(4):467-72.

Walker O, Salako LA, Risquat, A. Comparative trial of im artemether (ARTM) and sulfadoxine-pyrimethamine (S-P) in severe falciparum malaria in Nigerian children. 5th International Congress of Infectious, Nairobi, 1990 (abstract 570).

Waller D, Krishna S, Craddock C, Brewster D, Jammeh A, Kwiatkowski D, Karbwang J, Molunto P. The pharmacokinetics properties of intramuscular quinine of Gambian children with severe falciparum malaria. Trans R Soc Trop Med Hyg. 1990;84:488-91.

Wandell M, Powell JR, Hager WD, Fenster PE, Graves PE, Conrad KA, et al. Effect of quinine on digoxin kinetics. Clin Pharmacol Ther. 1980;28:425-30.
Wang TY, Xu RC. Clinical studies of treatment of falciparum malaria with artemether, a derivative of qinghaosu. J Trand Chin Med. 1985;5:240-2.

Wang NS, Guo XB, Liu GD, Fu LC, Li GQ, Arnold A. Pharmacokinetics of the combination pyrimethamine with sulfadoxine and mefloquine (FANSIMEF) in Chinese volunteers and the relative bioavailability of a lacquered tablets. Chemotherapy. 1990a;36:177-84.

Wang TY, Ma YS, Zhang DS, Yang JP, Li MH. Phase II clinical trial on artemether injection in 250 cases of malaria due to Plasmodium falciparum, $P$. vivax or P. malariae. Med Pharm Yunnan. 1990b;114-6.

Wanwimolruk S, Denton JR. Plasma protein binding of quinine: binding to human serum albumin, alpha 1-acid glycoprotein and plasma from patients with malaria. J Pharm Pharmacol. 1992;44:806-11.

Wanwimolruk S, Sunbhanich M, Pongmarutai M, Patamasucon P. Effects of cimetidine and ranitidine on the pharmacokinetics of quinine. $\mathrm{Br} \mathrm{J}$ Clin Pharmacol. 1986;22:346-50.

Wanwimolruk S, Kaewvichit S, Tanthayaphinant O, Suwannarach C, Ranratnachai A. Lack of effect of oral contraceptive use on the pharmacokinetics of quinine. Br J Clin Pharmacol. 1991a;31:179-81.

Wanwimolruk S, Chalcroft S, Coville PF, Campbell AJ. Pharmacokinetics of quinine in young and elderly subjects. Trans R Soc Trop Med Hyg. 1991b;85:714-7.

Wanwimolruk S, Kang W, Coville PF, Viriyayudhakorn S, Thitiarchakul S. Marked enhancement by rifampicin and lack of effect of isoniazid on the elimination of quinine in man. Br J Clin Pharmacol. 1995a;40:87-91.

Wanwimolruk S, Wong SM, Zhang H, Coville PF, Walker RJ. Metabolism of quinine in man: identification of a major metabolite, and effects of smoking and rifampicin pretreatment. J Pharm Pharmacol. 1995b;47:957-63.

Ward SA, Edwards G, Orme ML, Breckenridge AM. Determination of primaquine in biological fluids by reverse-phase high performance liquid chromatography. J Chromatogr. 1984;305:239-43.

Ward SA, Mihaly GW, Edwards G, Looareesuwan S, Phillips RE, Chanthavanich $\mathrm{P}$, et al. Pharmacokinetics of primaquine in man. II. Comparison of acute vs chronic dosage in Thai subjects. Br J Clin Pharmacol. 1985a;19:751-5.

Ward SA, Mihaly GW, Edwards G. Pharmacokinetics of primaquine in man II. Comparison of acute vs chronic dosage in Thai subjects. Br J Clin Pharmacol. 1985b;19:751-5.

Warhurst DC. Antimalarial Drugs An Update. Drugs. 1987;33:50-65.

Watt G, Long GW, Padre LP, Alban P, Sangalang R, Ranoa CP. Chloroquine and quinine: a randomised, doubleblind comparison of efficacy and side effects in the treatment of Plasmodium falciparum malaria in patients. Trans R Soc Trop Med Hyg. 1988;82:205-8.

Watt G, Shanks GD, Phintuyothin P. Prognostic significance of rise in parasitaemia during treatment of falciparum malaria. Trans $\mathrm{R}$ Soc Trop Med Hyg. 1992;86:359-60. 
Wattanagoon Y, Phillips RE, Warrell DA, Silamut K, Looareesuwan S, Nagachinta B, Back DJ. Intramuscular loading dose of quinine for falciparum malaria: pharmacokinetics and toxicity. BMJ. 1986;293:11-3.

Weidekammm E, Schwartz DE, Dubach UC, Webber B. Single dose investigation of possible interaction between the components of the antimalarial combination Fansimef ${ }^{\mathrm{R}}$. Chemotherapy. 1987;33:259-65.

Weinke T, Held T, Trautmann M, Rogler G, Mravak S, Alexander M, Pohle HD. Malaria therapy in 452 patients, with special reference to the use of quinine. J Infect. 1992;35:173-80.

Weinstein AJ, Gibbs RS, Gallagher M. Placental transfer of clindamycin and gentamicin in term pregnancy. Am J Obstet Gynecol. 1976;124:688-91.

Welling PG, Koch PA, Lau CC, Craig WA. Bioavailability of tetracycline and doxycycline in fasted and nonfasted subjects. Antimicrob Agents Chemother. 1977;11:462-9.

Wenk RE, Gebhardt FC, Bhagavan BS, Lustgarten JA, McCarthy EF. Tetracycline-associated fatty liver of pregnancy, including possible pregnancy risk after chronic dermatologic use of tetracycline. J Reprod Med. 1981;26:135-41.

Wennerholm A, Nordmark A, Pihlsgard M, Mahindi M, Bertilsson L, Gustafsson LL. Amodiaquine, its desethylated metabolite, or both, inhibit the metabolism of debrisoquine (CYP2D6) and losartan (CYP2C9) in vivo. Eur $\mathrm{J}$ Clin Pharmacol. 2006;62:539-46.

Westfall LK, Mintzer DL, Wiser TH. Potentiation of warfarin by tetracycline. Am J Hosp Pharm. 1980;37:1620-5.

Wetsteyn JC, De Vries PJ, Oosterhuis B, Van Boxtel CJ. The pharmacokinetics of three multiple dose regimens of chloroquine: implications for malaria chemoprophylaxis. Br J Clin Pharmacol. 1995;39:696-9.

Whelton A, Schach von Wittenau M, Twomey TM, Walker WG, Bianchine JR. Doxycycline pharmacokinetics in the absence of renal function. Kidney Int. 1974;5:365-71.

Whitby M, Wood G, Veenendaal JR, Rieckmann K. Chloroquine-resistant Plasmodium vivax (letter). Lancet. 1989;2:1395.

White NJ. Combination treatment for P. falciparum prophylaxis. Lancet. 1987;332:680-1.

White NJ. Antimalarial pharmacokinetics and treatment regimens. Br J Clin Pharmacol. 1992;34:1-10.

White NJ. Primaquine to prevent transmission of falciparum malaria. Lancet Infect Dis. 2013;13:175-81.

White NJ, Khrisna S. Treatment of malaria: some considerations and limitations of the current methods of assessment. Trans R Soc Trop Med Hyg. 1989;83:767-77.

White NJ, Looareesuwan S, Warrell DA, Warrell MJ, Bunnag D, Harinasuta T. Quinine pharmacokinetics and toxicity in cerebral and uncomplicated falciparum malaria. Am J Med. 1982;73:564-72.

White NJ, Chanthavanich P, Krishna S, Bunch C, Silamut K. Quinine disposition kinetics. Br J Clin Pharmacol. 1983a; 16:399-403.

White NJ, Looareesuwan S, Warrell DA, Warrell MJ, Chanthavanich P, Bunnag D, Harinasuta T. Quinine loading dose in cerebral malaria. Am J Trop Med Hyg. 1983c;32(1):1-5.

White NJ, Watt G, Bergqvist Y, Njeilesani EK. Parenteral chloroquine for treating falciparum malaria. J Infect Dis. 1987;155(2):192-201.

White NJ, Miller KD, Churchill FC, Berry C, Bown J, Williams BS. Chloroquine treatment of severe malaria in children. Pharmacokinetics, toxicity, and new dosage recommendations. New Engl $J$ Med. 1988;319:1493-500.

White NJ, Krishna S, Waller D, Craddock C, Kwiatkawski D, Brewster B. Open comparison of intramuscular chloroquine and quinine in children with severe chloroquinesensitive falciparum malaria. Lancet. 1989;334:1313-6.

White NJ, Waller D, Crawley J. Comparison of artemether and chloroquine for severe malaria in Gambian children. Lancet. 1992;339:317-21.

White NJ, van Vugt M, Ezzet F. Clinical pharmacokinetics and pharmacodynamics and pharmacodynamics of artemether-lumefantrine. Clin Pharmacokinet. 1999;37:105-25.

White NJ, Qiao LG, Qi G, Luzzatto L. Rationale for recommending a lower dose of primaquine as a Plasmodium falciparum gametocytocide in populations where G6PD deficiency is common. Malar J. 2012;11:e418.

White NJ, Pukrittayakamee S, Hien TT, Faiz MA, Mokuolu OA, Dondorp AM. Malaria. Lancet. 2013;383:723-35.

WHO. Resistance of malaria parasites to drugs. WHO Technical Report Series No. 296; 1965: Geneva, Switzerland. WHO Technical Report Series No. 375; 1967. p. 42.

William SB, Patchen LC, Churchill FC. Analysis of blood and urine samples for hydroxychloroquine and three major metabolites by high-performance liquid chromatography with fluorescence detection. J Chromatogr. 1988;433:197-206.

Wilson T, Edeson JFB. Studies on the chemotherapy of malaria, III. The treatment of acute malaria with chloroquine. Med J Malaya. 1954;9:115-31.

Win K, Thwe Y, Than KM. A clinical experience with artemether. Bur Med J. 1985;31(1):26-34.

Win K, Than M, Thwe Y. Comparison of combinations of parenteral artemisinin derivatives plus oral mefloquine with intravenous quinine plus oral tetracycline for treating cerebral malaria. Bull World Health Organ. 1992;70:777-82.

Winek CL, Davis ER, Collom WD, Shanor SP. Quinine fatality-case report. Clin Toxicol. 1974;7(2):129-32. 
Winstanley PA, Simooya O, Kofi-Ekue JM, Walker O, Salako LA, Edward G, et al. The disposition of amodiaquine in Zambians and Nigerians with malaria. Br J Clin Pharmacol. 1990;29:695-701.

Winstanley P, Newton C, Watkins W, Mberu E, Ward S, Warn P, Mwangi I, Waruiru C, Pasvol G, Warrell D, Marsh K. Towards optimal regimens of parenteral quinine for young African children with cerebral malaria: the importance of unbound quinine concentration. Trans R Soc Trop Med Hyg. 1993;87:201-6.

Wong RP, Salman S, Ilett KF, Siba PM, Mueller I, Davis TM. Desbutyllumefantrine is a metabolite of lumefantrine with potent in vitro antimalarial activity that may influence artemether-lumefantrine treatment outcome. Antimicrob Agents Chemother. 2011;55:1194-8.

Woo E, Greenblatt DJ. Pharmacokinetic and clinical implications of quinidine protein binding. J Pharm Sci. 1979;68(4):466-9.

World Health Organization. Division of Control of Tropical Diseases. Severe and complicated malaria. Trans R Soc Trop Med Hyg. 1990;84(Suppl 2):61-5.

World Health Organization. Guidelines for the treatment of malaria. 2nd ed. Geneva: World Health Organization; 2010.

World Health Organization, Division of Control of Tropical Diseases. Guidelines for the treatment of malaria. 3rd ed. Geneva, Switzerland: World Health Organization, 2015.

World Health Organization, Division of Control of Tropical Diseases. World Malaria report 2016. 2016.

World Health Organization, Division of Control of Tropical Diseases. The cardiotoxicity of antimalarials. Malaria Policy Advisory Committee meeting 22-24 March 2017, Geneva, Switzerland Background document for session 2 .

Yahya AM, McElnay JC, D'Arcy PF. Investigation of chloroquine binding to plastic materials. Int J Pharm. 1986;34:137-43.
Yavo W, Faye B, Kuete T, Djohan V, Oga SA, Kassi RR, et al. Multicentric assessment of the efficacy and tolerability of dihydroartemisinin-piperaquine compared to artemether-lumefantrine in the treatment of uncomplicated Plasmodium falciparum malaria in sub-Saharan Africa. Malar J. 2011;10:198.

Yosselson-Superstine S, Yanuka Y, Ishai S. Relationship between quinidine concentrations measured in saliva and erythrocytes, and in serum. Int J Clin Pharmacol Ther Toxicol. 1982;20(4):181-6.

Young MD. Failure of chloroquine and amodiaquine to suppress Plasmodium falciparum. Trans R Soc Trop Med Hyg. 1962;56:252.

Zhao S. High performance liquid chromatographic determination of artemisinin (qinghaosu) in human plasma and saliva. Analyst. 1987;112:661-4.

Zhao XJ, Ishizaki T. Metabolic interactions of selected antimalarial and nonantimalarial drugs with the major pathway (3-hydroxylation) of quinine in human liver microsomes. Br J Clin Pharmacol. 1997;44:505-11.

Zhao XJ, Ishizaki T. A further interaction study of quinine with clinically important drugs by human liver microsomes: determinations of inhibition constant (Ki) and type of inhibition. Eur J Drug Metab Pharmacokinet. 1999;24:272-8.

Zhou ZM, Anders JC, Chung H, Theoharides AD. Analysis of artesunic acid and dihydroqinghaosu on blood by high performance liquid chromatography with reductive electrochemical detection. J Chromatogr. 1987;414:77-90.

Zhou ZM, Huang YX, Xie GH, Sun XM, Wang YL, Fu LC, Jian HX, Guo XB, Li GQ. HPLC with Polarographic detection of artemisinin and it derivatives and application of the method to the pharmacokinetic study of artemether. J Liq Chromatogr. 1988;11(5):1117-37.

Zoest AR, Wanwimolruk S, Hung TC. Simple highperformance liquid liquid chromatographic method for the analysis of quinine in human plasma without extraction. J Liq Chromatogr. 1990;13:3481-91. 